\title{
Ar... I $2_{2}$ : A model system for complex dynamics
}

\author{
Alexei Buchachenko ${ }^{1}$, \\ Nadine Halberstadt ${ }^{2 *}$ \\ Bruno Lepetit ${ }^{3}$, \\ Octavio Roncero ${ }^{4}$ \\ ${ }^{1}$ Laboratory of Molecular Structure and Quantum Mechanics, \\ Department of Chemistry, Moscow State University, 119992 Moscow, Russia. \\ 2 LPQT-IRSAMC, Université Paul Sabatier and CNRS, \\ 118 route de Narbonne, 31062 Toulouse, France \\ ${ }^{3}$ LCAR-IRSAMC, Université Paul Sabatier and CNRS, \\ 118 route de Narbonne, 31062 Toulouse, France \\ ${ }^{4}$ Instituto de Matemáticas y Física Fundamental, \\ C.S.I.C., Serrano 123, 28006 Madrid, Spain.
}

December 4, 2002

${ }^{*}$ E-mail: nhalbers@irsamc.ups-tlse.fr 


\begin{abstract}
We review spectroscopic and photodissociation dynamical studies in the region of

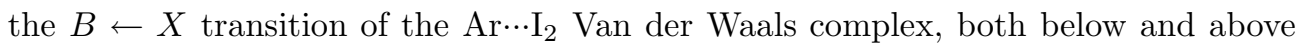
the dissociation limit of the $B\left({ }^{3} \Pi 0_{u}^{+}\right)$state. This very simple system constitutes a prototype for a wide range of molecular processes: vibrational predissociation involving intramolecular vibrational relaxation, electronic predissociation, cage effect... Each of these processes has been or still is the subject of differing interpretations: intramolecular vibrational relaxation involved in the vibrational predissociation of this system can be in the sparse or statistical regime, vibrational and electronic predissociation are in competition, and a direct, ballistic interpretation of the cage effect as well as a nonadiabatic one have been proposed. The study of the dependence of these dynamical processes on the relative orientation of the two partners of the complex (stereodynamics) is made possible by the coexistence of two stable $\operatorname{Ar} \cdots \mathrm{I}_{2}(X)$ isomers. Experimental as well as theoretical results are reviewed. Experiments range from frequency-resolved to time-dependent studies, including the determination of final state distributions. Theoretical studies involve potential energy surface calculations for several electronic states of the complex and their couplings, and adiabatic as well as nonadiabatic dynamical simulations.
\end{abstract}




\section{Introduction}

One of the main goals in chemical physics is to understand energy transfer processes and to be able to predict the properties and dynamical behavior of a molecular system. Van der Waals complexes constitute ideal model systems from that point of view. Because of the weakness of the intermolecular bond, the partners building the complex retain their identity and energy transfers are thus easily identified. Their dissociation represents the second half of a collision with a limited range of impact parameters, which allows one to make fruitful comparisons with collisional results. Finally, studying the dependence of the energy redistribution and fragmentation processes on the size of the cluster may help bridge the gap with condensed phase dynamics.

The simplest of the Van der Waals complexes for studying energy transfers are built with a rare gas atom and a diatomic molecule. Among them, Ar $\cdots \mathrm{I}_{2}$ has received special attention. It exhibits very rich dynamics, with processes ranging from vibrational predissociation (VP), intramolecular vibrational relaxation (IVR), electronic predissociation (EP), and even geminate recombination or "caging", a typical effect usually observed in condensed phase. The interpretation of these processes has lead to many puzzles, controversies, and surprises. They mainly originate from the fact that different energy transfer and decay pathways are often in competition, so that it is difficult to distinguish between them in experiments. From the theoretical point of view, competing processes should be taken into account simultaneously, which can make the problem computationally untractable. In addition, it is still nowadays quite a challenge to calculate $a b$ initio the potential energy surfaces (PES) and couplings involved in the dynamics of this system. This has made Ar $\cdots \mathrm{I}_{2}$ a typical example where experiments raise a new theoretical interpretation, 
which is in turn tested by new experiments which can result in another interpretation, and so on until consistent and unambiguous agreement is reached. Hence Ar... $\mathrm{I}_{2}$ constitutes a benchmark system for comparisons between calculations and experiments.

The aim of this review is to summarize the vast amount of studies on the $\operatorname{Ar}+\mathrm{I}_{2}$ system, identify their most important implications to general understanding of energy transfer phenomena, describe the current state of interpretation and controversy, and draw the perspectives for future works. We have taken the point of view of considering Ar $\cdots \mathrm{I}_{2}$ as a prototypical system to study different dynamical processes, as we believe that insight gained from these studies is more general and valuable than the particular results obtained for this specific system. Therefore, the different aspects of the Ar $\cdots \mathrm{I}_{2}$ structure and the different dynamical processes are discussed in separate sections, although this presentation brings some unavoidable repetitions. For the same reason, although this review is mainly devoted to the Ar... I 2 Van der Waals complex, references to other works in related fields (collisional energy transfer in the iodine molecule, caging in an inert gas matrix, structure and dynamics of related complexes, etc.) are also given when appropriate.

After a historical overview in section 2, section 3 introduces the relevant potential energy curves of the iodine molecule and details the advances made in the determination of the $\mathrm{Ar} \cdots \mathrm{I}_{2}$ interactions in the ground and excited electronic states, as well as the couplings between the electronic states of the bare molecule induced by the presence of the argon atom. Section 4 presents the cage effect in $\mathrm{Ar} \cdots \mathrm{I}_{2}$ with its different interpretations including the one now accepted, and compares with the geminate recombination of $\mathrm{I}_{2}$ in an argon matrix. Section 5 introduces the specific features of the vibrational predissociation process in $\mathrm{Ar} \cdots \mathrm{I}_{2}$, presents the different interpretations of intramolecular vibrational relaxation in this system, and compares the behavior of the T-shaped and linear isomers. 
In section 6 , the origin of the electronic predissociation process is discussed, as well as its possible interferences with the competing VP-IVR process. Finally, section 7 concludes and sketches perspectives for solving the open problems in this old, but still not fully understood, prototype system.

\section{Historical overview}

\subsection{Collisional Ar $+\mathbf{I}_{2}$ system}

There is a considerable amount of literature on the collisional relaxation of excited $\mathrm{I}_{2}$, which has been nicely summarized by Krajnovich et al. (Krajnovich et al., 1989a). The beginning of this long history may be traced back to Wood, who, in 1911 (Wood, 1911a,b), measured the reduction of fluorescence intensity of an $\mathrm{I}_{2}$ cell exposed to sun light as a function of the pressure of added gases, and showed that the fluorescence spectra were gradually evolving from discrete to band spectra as the pressure of the rare gas increased in the cell (Franck and Wood, 1911).

A correct interpretation of these pioneering results was not possible until the advent of quantum mechanics. It is now known that $\mathrm{I}_{2}$ visible absorption is related to the $B\left({ }^{3} \Pi 0_{u}^{+}\right) \leftarrow X\left({ }^{1} \Sigma 0_{g}^{+}\right)$electronic transition. Fluorescence quenching of the $\mathrm{I}_{2}(B)$ excited state by foreign gases is related to electronic transitions from the $B$ state to repulsive electronic states (see sections 3.1, 3.5, and 6) induced by interaction with a colliding atom. This leads to the fragmentation of the $\mathrm{I}_{2}$ molecule in two hot I atoms which cannot fluoresce. The qualitative changes in the fluorescence spectra are related to vibrational and rotational transitions induced by collision between $\mathrm{I}_{2}(B)$ and the foreign gas.

During several decades, experiments on the subject remained qualitative because they 
were produced on photographic plates (Rössler, 1935; Arnot and McDowell, 1958). Quantitative fluorescence spectra of $\mathrm{I}_{2}(B)$ and its quenching by Ar became possible with the advent of photomultipliers, as first obtained by Klemperer and his group (Brown and Klemperer, 1964; Steinfeld and Klemperer, 1965), followed by others (Kurzel and Steinfeld, 1970; Capelle and Broida, 1973; Nakagawa et al., 1986), and became more systematic with the use of tunable laser sources (Capelle and Broida, 1973; Nakagawa et al., 1986). The dependence of the quenching cross section on the initial vibrational excitation $v^{\prime}$ was found to be rather smooth, with an average quenching cross section of the order of $5 \AA^{2}$ (Capelle and Broida, 1973). Vibrational energy transfer is more efficient as $v^{\prime}$ increased (Steinfeld and Klemperer, 1965), and less efficient than rotational energy transfer (Rubinson et al., 1974a).

Although the global picture for these phenomena is clear, a detailed theoretical interpretation of these results was slow to emerge, and is still nowadays not fully satisfactory. The quenching cross section was found to be proportional to the polarisability of the target and to the duration of the collision, hence to the square root of the reduced mass of the system (Rössler, 1935; Selwyn and Steinfeld, 1969). Two independent models were developed (Selwyn and Steinfeld, 1969; Thayer and Yardley, 1972), based on a perturbative treatment of the instantaneous dipole-dipole interaction, but differing in the definition of the final state after quenching. According to Selwyn and Steinfeld (Selwyn and Steinfeld, 1969), the quencher can be in any final state, in which case the instantaneous dipole couples at the first order of perturbation theory the $\mathrm{I}_{2}$ initial $B\left({ }^{3} \Pi 0_{u}^{+}\right)$state with final states which have a $g / u$ symmetry opposite to the initial one $\left(a 1_{g}, a^{\prime} 0_{g}^{+}\right)$. On the other hand, Thayer and Yardley (Thayer and Yardley, 1972) assumed similar initial and final states for the quencher, in which case the final states of $\mathrm{I}_{2}$ must have the same $g / u$ symmetry as 
the initial $B$ state and the quenching could be attributed to the $0_{u}^{-}$state (Nakagawa et al., 1986) . This contradiction could in principle be solved by looking at the dependence of the quenching cross section as a function of the initial vibrational state, which is related to the Franck-Condon factors between the initial bound state and the final continuum state. Unfortunately, the quenching rate was found to be a smooth function of the initial excitation (Nakagawa et al., 1986; Capelle and Broida, 1973). This could result from competition with collisional vibrational energy transfer (Tellinghuisen, 1985). Selection of the initial state had to wait for the advent of low temperature supersonic beams, where Van der Waals complexes are formed in well defined initial states and can undergo processes like vibrational or electronic predissociation closely related to vibrational energy transfers and quenching in collisional conditions.

\subsection{The $\mathrm{Ar} \cdots \mathrm{I}_{2}$ Van der Waals complex}

The first experimental evidence of the existence of the Ar $\cdots \mathrm{I}_{2}$ Van der Waals complex was obtained by Levy and his team (Kubiak et al., 1978; Levy, 1981). In a series of pioneering experiments (Smalley et al., 1976; Kubiak et al., 1978; Johnson et al., 1978; Sharfin et al., 1979; Kenny et al., 1980a,b; Blazy et al., 1980; Johnson et al., 1981; Levy, 1981), Levy and co-workers have studied the spectroscopy and dynamics of $\mathrm{I}_{2}$ Van der Waals complexes in a supersonic expansion, via the $B \leftarrow X$ transition. After a first unsuccessful attempt

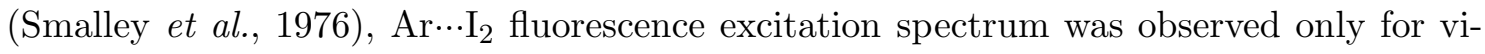
brational levels of $\mathrm{I}_{2}(B)$ higher than $v^{\prime}=12$. The laser induced fluorescence intensity was found to be an oscillatory function of the $\mathrm{I}_{2}$ vibrational excitation. This behavior was interpreted as the result of the competition between vibrational (VP) and electronic (EP) 
predissociation :

$$
\begin{array}{lll}
\operatorname{Ar} \cdots \mathrm{I}_{2}\left(B, v^{\prime}\right) & \stackrel{\mathrm{VP}}{\longrightarrow} & \mathrm{Ar}+\mathrm{I}_{2}\left(B, v \leq v^{\prime}\right) \\
\operatorname{Ar} \cdots \mathrm{I}_{2}\left(B, v^{\prime}\right) & \stackrel{\mathrm{EP}}{\longrightarrow} & \mathrm{Ar}+\mathrm{I}\left({ }^{2} P_{3 / 2}\right)+\mathrm{I}\left({ }^{2} P_{3 / 2}\right) .
\end{array}
$$

Since channel (1) produces electronically excited $I_{2}$ fragments which can fluoresce while channel (2) is dark, measurements of the $\mathrm{I}_{2}$ fluorescence quantum yield in conjunction with the $\mathrm{Ar} \cdots \mathrm{I}_{2}$ absorption spectrum can provide the relative importance of vibrational and electronic predissociation. For $v^{\prime}<12$ electronic predissociation dominates, which explains why no fluorescence could be detected.

Goldstein et al. (Goldstein et al., 1986) measured the relative quantum yields for $\operatorname{Rg} \cdots \mathrm{I}_{2}$ complexes, with $\mathrm{Rg}=\mathrm{He}, \mathrm{Ar}, \mathrm{Kr}$, and Xe. In agreement with the results of Levy and co-workers (Kubiak et al., 1978), they observed no significant variation of the quantum yield as a function of the excited vibrational state for $\operatorname{He} \cdots \mathrm{I}_{2}\left(B, v^{\prime}\right)$, while for $\operatorname{Ar} \cdots \mathrm{I}_{2}\left(B, v^{\prime}\right)$ an oscillatory behaviour with $v^{\prime}$ was confirmed. For $\mathrm{Kr} \cdots \mathrm{I}_{2}$ complexes the extremely low fluorescence quantum yield measured (Goldstein et al., 1986) indicates that the rates associated to VP and EP become comparable only for $v^{\prime}$ values close to the $\mathrm{I}_{2}(\mathrm{~B})$ dissociative threshold. For complexes of Xe or more than one Ar atom, however, no fluorescence was detected, clearly indicating that EP becomes the dominant channel. Therefore, Ar $\cdots \mathrm{I}_{2}$ is an ideal system to study the competition between VP and EP. Whether the oscillations of the fluorescence intensity are due to electronic or vibrational predissociation is still a subject of debate. A related question is whether VP dynamics, which is mediated by Intramolecular Vibrational Relaxation (IVR), is in a sparse or statistical regime. Another issue is the nature of the electronic state(s) responsible for electronic predissociation. This points will be discussed in sections 5 and 6 . 


\subsection{Cage effect}

Another puzzling effect was found in $\mathrm{Ar} \cdots \mathrm{I}_{2}$. When excited above the $B$ state dissociation limit, complexed $\mathrm{I}_{2}$ still exhibits some fluorescence, whereas uncomplexed $\mathrm{I}_{2}$ is $100 \%$ dissociated at the same wavelength. This means that the presence of one sole argon atom can induce the recombination of the departing $\mathrm{I}$ atoms into $\mathrm{I}_{2}$. This process is reminiscent of a well-known condensed phase process, the so-called "cage effect".

The condensed phase "cage effect" is a process in which two dissociating fragments recombine in situ by colliding with atoms or molecules of the surrounding solvent (the "cage"). It is also called "geminate recombination" as opposed to recombination after diffusion, and was introduced by Franck and Rabinovitch (Franck and Rabinovitch, 1934) to explain the reduced photochemical yield of free radicals in solutions as compared to the gas phase. Since then, it has played a central role in the reactive photodynamics studies in condensed media. These include photodissociation studies of small molecules in Van der Waals solids and clusters (Chergui and Schwentner, 1992), liquid phase (Harris et al., 1988) and high pressure (Schroeder and Troe, 1987) gases, by time-independent experiments and by molecular dynamics simulations, as well as more recent femtosecond time-resolved measurements in clusters (Papanikolas et al., 1992, 1993), in liquids (Zewail et al., 1992; Schwartz et al., 1993; Alfano et al., 1992), in high pressure gases (Lienau and Zewail, 1994), and in solids (Zadoyan et al., 1997; Apkarian and Schwentner, 1999; Pedersen and Weitz, 2002). In many of these studies, $I_{2}$ is the prototype molecule to investigate the photodissociation-recombination process.

Molecular clusters offer a unique environment to study cage effects, since the size of the solvent cage surrounding a chromophore can potentially be controlled, allowing one to 
study the effect of increasing solvation on reaction dynamics (Fei et al., 1992; Castleman, Jr., 1992; Papanikolas et al., 1993; Liu et al., 1993; Hu and Martens, 1993b; Gerber et al., 1994; Jungwirth et al., 1996; Greenblatt et al., 1997; Delaney et al., 1999; Žd̆ánská et al., 2000; Baumfalk et al., 2001). The interpretation of the cage effect in $\mathrm{Ar} \cdots \mathrm{I}_{2}$ has led to several rebounds, from a purely kinematic or "ballistic" to a nonadiabatic process. A major surprise came in the interpretation of a crucial experiment designed to distinguish between the possible mechanisms (Burke and Klemperer, 1993b). The conclusion of that experiment was that two isomers must coexist in supersonic expansions, a perpendicular one recognized early on and a linear one that was conjectured to explain the results. Its existence was later confirmed, and has led to a new series of experimental and theoretical studies to examine the dependence of the different processes on the initial geometry of the complex. The study of the cage effect in $\mathrm{Ar} \cdots \mathrm{I}_{2}$ is detailed in section 4, while a comparative study of the VP/EP fragmentation dynamics from the two isomers will be presented in section 5 .

It emerges from this historical survey that although the Ar $\cdots \mathrm{I}_{2}$ Van der Waals complex contains only a diatomic molecule and a rare gas atom, it can be seen as a prototype to study a wide range of molecular physics processes, from vibrational predissociation involving complex IVR dynamics, to the typical condensed phase cage effect, through electronic predissociation and its competition with IVR-VP. And, last but not least, these processes can be studied in two different geometries, corresponding to the linear and Tshaped isomers of $\mathrm{Ar} \cdots \mathrm{I}_{2}$. 


\section{Structure, energetics, and potential energy surfaces}

The range of dynamical processes present in $\mathrm{Ar} \cdots \mathrm{I}_{2}$, as well as their complexity, requires an accurate description of the $\mathrm{Ar}-\mathrm{I}_{2}$ interaction in the ground and excited electronic states of $\mathrm{I}_{2}$, as well as that of the couplings between these states induced by the argon. This section describes and comments the various approaches that have been used up to now to tackle this difficult task.

First, we briefly summarize the useful information on the structure of the isolated $\mathrm{I}_{2}$ molecule. Second, we go into the details of the various pieces of information gained from different experimental measurements. Then the empiricial, semiempirical and $a b$ initio

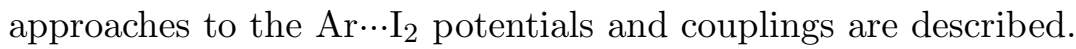

\subsection{Electronic structure of the $\mathbf{I}_{2}$ molecule}

The valence states of the iodine molecule form the lowest manifold of the molecular terms correlating with the $\mathrm{I}\left({ }^{2} \mathrm{P}\right)+\mathrm{I}\left({ }^{2} \mathrm{P}\right)$ dissociation limit. When spin-orbit (SO) interaction is taken into account, the asymptotic threshold splits into three limits $\mathrm{I}\left({ }^{2} \mathrm{P}_{3 / 2}\right)+\mathrm{I}\left({ }^{2} \mathrm{P}_{3 / 2}\right)$, $\mathrm{I}\left({ }^{2} \mathrm{P}_{3 / 2}\right)+\mathrm{I}^{*}\left({ }^{2} \mathrm{P}_{1 / 2}\right)$, and $\mathrm{I}^{*}\left({ }^{2} \mathrm{P}_{1 / 2}\right)+\mathrm{I}^{*}\left({ }^{2} \mathrm{P}_{1 / 2}\right)$, separated by the atomic $\mathrm{SO}$ splitting $\Delta=7602.98 \mathrm{~cm}^{-1}$. The detailed description of the valence states for iodine goes back to the classical works of Mulliken $(1957 ; 1971)$. It was shown that their structure obeys Hund's case (c) coupling scheme with $\Omega_{w}^{\sigma}$ classification, where $\Omega$ is the projection of the total (orbital plus spin) electronic angular momentum on the $\mathrm{I}_{2}$ axis $\mathbf{r}, \sigma= \pm 1$ and $w=u, g$ being the parities with respect to coordinate inversion (reflection of the electronic coordinates through the a plane containing the molecular axis) and nuclear permutation (inversion of electronic coordinates in the body-fixed frame), respectively. 
The valence manifold consists of 23 levels (36 states, 13 levels being doubly degenerate), namely, $X 0_{g}^{+}, a^{\prime} 0_{g}^{+}, 30_{g}^{+}, 40_{g}^{+}, 0_{g}^{-}, B 0_{u}^{+}, B^{\prime} 0_{u}^{-}, 20_{u}^{-}, 30_{u}^{-}, 40_{u}^{-}, a 1_{g}, 21_{g}, 31_{g}, A 1_{u}, B^{\prime \prime} 1_{u}$, $31_{u}, 41_{u}, 51_{u}, 12_{g}, 22_{g}, A^{\prime} 2_{u}, 22_{u}$, and $3_{u}$, where the most common spectroscopic notations for iodine are used (Huber and Herzberg, 1979).

They originate from 12 non-relativistic precursors in Hund's case (a) ${ }^{2 S+1} \Lambda_{w}^{\sigma}$ classification, (where $\Lambda$ and $\Sigma$ are the projections on the molecular axis of the electronic orbital and spin angular momenta, respectively) $1^{1} \Sigma_{g}^{+}\left(X 0_{g}^{+}\right), 2^{1} \Sigma_{g}^{+}\left(40_{g}^{+}\right),{ }^{1} \Sigma_{u}^{-}\left(30_{u}^{-}\right),{ }^{1} \Pi_{g}\left(21_{g}\right)$, ${ }^{1} \Pi_{u}\left(B^{\prime \prime} 1_{u}\right),{ }^{1} \Delta_{g}\left(22_{g}\right),{ }^{3} \Sigma_{g}^{-}\left(30_{g}^{+}, 31_{g}\right), 1^{3} \Sigma_{u}^{+}\left(20_{u}^{-}, C 1_{u}\right), 2^{3} \Sigma_{u}^{+}\left(41_{u}, 40_{u}^{-}\right),{ }^{3} \Pi_{g}\left(12_{g}\right.$, $\left.a 1_{g}, 0_{g}^{-}, a^{\prime} 0_{g}^{+}\right),{ }^{3} \Pi_{u}\left(A^{\prime} 2_{u}, A 1_{u}, B^{\prime} 0_{u}^{-}, B 0_{u}^{+}\right)$, and ${ }^{3} \Delta_{u}\left(3_{u}, 22_{u}, 51_{u}\right)$.

The literature provides a wealth of information on the valence potential energy curves. Empirical curves are available for ten of these states (for brief account see, e.g., (Buchachenko and Stepanov, 1996b; Pazyuk et al., 2001)). In addition, two high-level relativistic ab initio calculations on the complete set of $\mathrm{I}_{2}$ electronic states have been performed (Teichteil and Pélissier, 1994; de Jong et al., 1997).

Since the $X\left({ }^{1} \Sigma 0_{g}^{+}\right)$and $B\left({ }^{3} \Pi 0_{u}^{+}\right)$states are of prime interest in the present context, it is worth referring to the accurate empirical Rydberg-Klein-Rees (RKR) potential curves (Martin et al., 1986; Gerstenkorn and Luc, 1985). The $B$ state correlates with the I + I* asymptotic limit, and intersects repulsive or weakly-bound electronic states $\left(B^{\prime \prime} 1_{u}, 12_{g}\right.$, $a 1_{g}, a^{\prime} 0_{g}^{+}, 20_{u}^{-}$, and $3_{u}$ ) going to the ground $\mathrm{I}+\mathrm{I}$ asymptote, as illustrated in Fig. 1. These states are responsible for various electronic predissociation processes of $\mathrm{I}_{2}(B)$ (Katô and Baba, 1995), which are very slow in isolated $\mathrm{I}_{2}$.

Above the valence states there is a manifold of so-called ion-pair states correlating with the $\mathrm{I}^{+}\left({ }^{3} \mathrm{P}_{j},{ }^{1} \mathrm{D}_{j}\right)+\mathrm{I}^{-}\left({ }^{1} \mathrm{~S}_{0}\right)$ dissociation limits. 


\subsection{Ar $\cdots \mathrm{I}_{2}(X, B)$ dissociation energies: The existence of two isomers}

The initial studies on vibrationally inelastic $\mathrm{Ar}+\mathrm{I}_{2}$ collisions did not bring any direct, accurate information on the $\mathrm{Ar}-\mathrm{I}_{2}$ interaction. Real progress was made in the late seventies by Levy and co-workers in their spectroscopic investigations of Van der Waals $\operatorname{Rg} \cdots \mathrm{I}_{2}$ complexes in a supersonic expansion via the $B\left({ }^{3} \Pi 0_{u}^{+}\right) \leftarrow X\left({ }^{1} \Sigma 0_{g}^{+}\right)$transition (Smalley et al., 1976; Kubiak et al., 1978; Sharfin et al., 1979; Kenny et al., 1980a; Blazy et al., 1980; Johnson et al., 1981; Levy, 1981), see section 2. Excitation spectroscopy was supplemented by dispersed fluorescence measurements which probed the final vibrational state distribution $v$ of the VP products (Blazy et al., 1980). For excitations to $v^{\prime}<30$, VP proceeds through the transfer of $\Delta v=v^{\prime}-v=-3$ quanta. For $v^{\prime}=30$, the $\Delta v=-3$ product disappears and VP proceeds through the transfer of four vibrational quanta, due to the anharmonicity of the $\mathrm{I}_{2}$ vibration which reduces the vibrational quantum energy when $v$ increases. This allowed Blazy et al. (1980) to firmly establish both upper and lower limits for $D_{0}$ of $\operatorname{Ar} \cdots \mathrm{I}_{2}: D_{0}(B)=223 \pm 3 \mathrm{~cm}^{-1}$. The detection of additional spectral bands assigned to $B$-state excited Van der Waals levels (intermolecular stretching and double bending excitation) made it possible to estimate the vibrational frequencies and zero-point energy, and to deduce the binding energy $D_{e}(B)$. The dissociation energy in the $X$ state was determined from the (blue) frequency shift of the complexed vs uncomplexed I transition: $D_{0}(X)=237 \pm 3 \mathrm{~cm}^{-1}$. The estimations for binding energies are collected in Tables 1 and 2 .

At the time at which these results were obtained, there was no definitive structural information available for the $\mathrm{Ar} \cdots \mathrm{I}_{2}$ complex. Ar $\cdots \mathrm{ClF}$ was known to be linear from microwave studies (Harris et al., 1974). However, the structure of $\mathrm{He} \cdots \mathrm{I}_{2}$ had been determined by Levy and coworkers (Smalley et al., 1978) to be T-shaped from analysis of rotationally 
resolved bands of the $B \leftarrow X$ transitions. The smaller rotational constants of $\mathrm{Ar} \cdots \mathrm{I}_{2}$ did not allow the same level of resolution, but it was assumed to be T-shaped in analogy with He $\cdots \mathrm{I}_{2}$. This geometry was given even more credit by subsequent studies of dihalogen-rare gas complexes characterized to be T-shaped from rotational analysis of the $B \leftarrow X$ transition, e.g., $\mathrm{Ne} \cdots \mathrm{Br}_{2}$ (Thommen et al., 1985), $\mathrm{Ne} \cdot \mathrm{Cl}_{2}$ (Evard et al., 1986), and $\mathrm{Ar} \cdots \mathrm{Cl}_{2}$ (Evard et al., 1988b), and from the microwave spectroscopy of $\mathrm{Ar} \cdots \mathrm{Cl}_{2}(X)$ (Xu et al., 1993). It was later confirmed by Burke and Klemperer (1993a) that the $B \leftarrow X$ spectrum could be fitted using a perpendicular geometry for Ar... $\mathrm{I}_{2}$.

The first accurate large-scale ab initio calculations performed for $\mathrm{Ar} \cdots \mathrm{Cl}_{2}(X)$ by Tao and Klemperer (1992) gave a quite unexpected result: two minima were found, a T-shaped and a linear one, the latter being significantly deeper. This could be rationalized in the following manner. In the $X$ state the $\sigma^{*}$ orbital of a dihalogen molecule is empty, which may result in a fairly short equilibrium bond length and hence a stronger binding energy for the linear isomer. This finding was later confirmed by numerous ab initio studies of the ground-state chlorine and bromine complexes (Chałasiński et al., 1994; Naumkin and Knowles, 1995; Williams et al., 1997; Rohrbacher et al., 1997a; Naumkin and McCourt, 1997, 1998a; Rohrbacher et al., 1999a,b; Cybulski and Holt, 1999; Naumkin and McCourt, 1999; Prosmiti et al., 2002a) and seemed to be in contradiction with experiment, which only detected T-shaped isomers.

This contradiction was solved by Huang et al. (1995) for $\mathrm{He} \cdots \mathrm{Cl}_{2}$. These authors demonstrated that the higher zero-point energy of the linear complex due to the doubly degenerate bending mode reversed the order of stability of the potential minima. Also, ab initio calculations on the $B$-state PES (Chałasiński et al., 1994; Cybulski et al., 1995; Rohrbacher et al., 1997b; Williams et al., 1999) proved that its minimum is T-shaped and 
that transitions from the ground-state linear isomer may fall in a continuum which could go unnoticed. Indeed, upon excitation to the $B$ state, a $\pi^{*}$ electron of $\mathrm{I}_{2}$ is transferred to the $\sigma^{*}$ orbital, thus increasing the bond length and lowering the binding energy in the linear configuration. The situation in heavier complexes is less certain, mainly because of the lack of rotational resolution in experimental spectra. There are strong indications that high-resolution spectra reveal the existence of linear isomers of $\mathrm{He}_{\cdots} \mathrm{Br}_{2}(X)$ and $\mathrm{Ne} \cdots \mathrm{I}_{2}(X)$ (Hernández et al., 2000; Buchachenko et al., 2002; Burroughs et al., 2001).

The first experimental evidence for the existence of a linear Ar $\cdots \mathrm{I}_{2}$ isomer was obtained by Burke and Klemperer (1993b). Using $B \leftarrow X$ fluorescence excitation spectroscopy they found that the quasidiscrete spectrum observed by Levy and co-workers (Blazy et al., 1980; Johnson et al., 1981) laid on the background of a quite intense continuous absorption. Quantitative measurements of the fluorescence quantum yield were carried out for the continuum part of the absorption (Burke and Klemperer, 1993b), to discriminate between two possible interpretations of the cage effect (see section 4). Burke and Klemperer concluded that the continuous absorption was due to a linear isomer which coexisted in the jet with the T-shaped one, with a population ratio estimated to be 3:1 (Burke and Klemperer, 1993b).

More evidence in support of this interpretation came from ab initio calculations discussed in subsection 3.6 and from experiments by the group of Donovan and Lawley (Cockett et al., 1993, 1994; Goode et al., 1994; Cockett et al., 1996) on the high-lying Rydberg states of the Ar $\cdots \mathrm{I}_{2}$ complex. Many of the vibrational bands in the resonance enchanced multiphoton ionization (REMPI) and zero electron kinetic energy (ZEKE) spectra of the Rydberg states converging to the ground state of the complex cation were split into doublets, (Cockett et al., 1993, 1994) subsequently assigned (Cockett et al., 1996) to 
transitions from the T-shaped and linear isomers.

Measurements of the vibrational distributions of the photofragmentation products allowed Stevens Miller et al. (1999) to determine the dissociation energy of the linear Ar $\cdots \mathrm{I}_{2}(X)$ isomer as $D_{0}(L, X)=172 \pm 4 \mathrm{~cm}^{-1}$. From their previous estimate of a $3: 1$ linear:T-shaped isomer population based on intensity measurements, these authors concluded that the linear isomer should be more stable than the T-shaped one by ca. $30 \mathrm{~cm}^{-1}$, assuming thermal equilibrium at the temperature of $15 \pm 5 \mathrm{~K}$. This yielded a dissociation energy for the latter of $D_{0}(T, X)=142 \pm 15 \mathrm{~cm}^{-1}$, hence of $D_{0}(T, B)=128 \pm 15 \mathrm{~cm}^{-1}$ for the excited state (Stevens Miller et al., 1999). These results contradict the determination of $D_{0}(T, B)=223 \pm 3 \mathrm{~cm}^{-1}$ by Levy's group (Blazy et al., 1980). Indeed, if the revised $B$-state dissociation energy is correct, the $\Delta v=-2 \mathrm{VP}$ channel should be open and dominant. Klemperer and coworkers suggested that the $\Delta v=-2$ channel could have been present in Levy's experiment but not detected. This could be due to the fact that $\Delta v=-2 \mathrm{VP}$ fragments are slow to separate since translational energy is very low for that channel, and fluorescence from $\mathrm{I}_{2}\left(v^{\prime}-2\right)$ could be quenched by the competing electronic predissociation process. Photofragmentation of the linear isomer is much faster, so that the corresponding vibrational product state distributions are unlikely to be affected by EP.

The value of $D_{0}(T, X)$ determined by anion photoelectron spectroscopy of $\mathrm{Ar}_{\cdots} \mathrm{I}_{2}^{-}$ (Asmis et al., 1998) combined with the available estimation of $\mathrm{Ar}_{\cdots} \mathrm{I}_{2}^{-}$dissociation energy (Naumkin and McCourt, 1999), $\mathrm{D}_{0}(T, X)=190 \pm 80 \mathrm{~cm}^{-1}$, did not resolve the controversy on Ar $\cdots \mathrm{I}_{2}$ energetics, since the error bars cover both Levy's and Klemperer's values.

In order to resolve this contradiction, Burroughs and Heaven (2001) implemented the optical-optical double resonance technique to measure the rotational $j$ distributions of 
the $\mathrm{I}_{2}(B)$ fragment after excitation of both the T-shaped and the linear isomers. The value $220 \mathrm{~cm}^{-1} \leq D_{0}(T, B) \leq 232 \mathrm{~cm}^{-1}$ was deduced from energy conservation at the highest value of $j$ observed, in perfect agreement with the result of Levy's group. These observations cannot completely rule out the assumption of Klemperer et al. since higher rotational channels could also be quenched by electronic predissociation, but the similarity of the $D_{0}(T, B)$ values adds more arguments in support of Levy's data.

Burroughs and Heaven (2001) suggested that another weak point in the deduction of the dissociation energies from intensity measurements was the assumption of thermal equilibrium in the beam. This point has been the subject of a thorough molecular dynamics simulation (Bastida et al., 2002) describing the collisional isomerization and cooling of $\operatorname{Ar} \cdots \mathrm{I}_{2}(X)$ in a free jet using the $\operatorname{CCSD}(\mathrm{T})$ PES by Kunz et al. (1998) described in 3.6. This simulation has reached the surprising conclusion that the populations of the two isomers remain in thermal equilibrium as the expansion proceeds. This is due to the fact that when an argon atom enters the potential well of an already formed Ar $\cdots \mathrm{I}_{2}$ complex, it acquires a kinetic energy much larger than its asymptotic value, which can make the complex overcome its isomerization barrier. Another mechanism was also put in evidence, in which a linearly incoming argon atom could replace the perpendicularly attached atom or vice-versa, the net effect being isomerization with the exchange of argon atoms: this mechanism was called "swap cooling", and could account for up to half of the coldest collisions.

Other possible sources of inaccuracy affecting the derivation of $D_{0}(X, T)$ are probably related to intensity ratio determination, namely, the problem of distinguishing the absorption from each isomer, different absorption probabilities, and the possible role of saturation effects (Klemperer, 2001). We come back to this point in section 5.3. 


\subsection{Empirical potentials}

Initially, the need for $\mathrm{Ar}-\mathrm{I}_{2}(X, B)$ interaction potentials arose from experiments on vibrationally-inelastic collisions [e.g. (Brown and Klemperer, 1964; Steinfeld and Klemperer, 1965; Kurzel and Steinfeld, 1970; Kurzel et al., 1971) and the literature database in (Steinfeld, 1984, 1987)]. These measurements performed under bulk conditions provided estimations for inelastic transition rate constants which do not allow straightforward inversion of the potential. Early theoretical interpretations (Kajimoto and Fueno, 1972; Rubinson et al., 1974b; Rubinson and Steinfeld, 1974) designed and used empirical potentials representing the total PES as a sum of atom-atom potentials (Hill, 1946; Kitaigorodskii, 1951). The addition of two identical potentials gives a PES with a T-shaped configuration and a saddle point in the collinear arrangement. The $\mathrm{Rg}-\mathrm{X}$ rare gas-halogen potentials were approximated by $\mathrm{Rg}-\mathrm{Rg}$ ' potentials from Hirschfelder et al. (1954), where Rg' is the rare gas atom following $\mathrm{X}$ in the periodic table, see, e.g., the work by Secrest and Eastes (1972), or by simple correlation rules.

A notable exception is the work of Rubinson et al. (1974b), where the parameters of a model Buckingham Rg-I potential were optimized by means of three-dimensional quasiclassical trajectory calculations to reproduce observed probabilities of vibrationallyinelastic transitions in $\mathrm{Rg}+\mathrm{I}_{2}(B)$ collisions. Equilibrium properties of these potentials are presented in Table 1, entry 16.

Later on, more collision experiments were carried on the vibrationally (Sulkes et al., 1980; Hall et al., 1983; Gentry, 1984; Baba and Sakurai, 1985; Rock et al., 1988; Krajnovich et al., 1989a,b; Ma et al., 1991; Du et al., 1991; Nowlin and Heaven, 1993; Lawrence et al., 1997) and rotationally (Dexheimer et al., 1982, 1983; Derouard and Sadeghi, 1984a,b) 
inelastic scattering, collisional line broadening (Drabe et al., 1985b,a; Drabe and van Voorst, 1985) and diffusion coefficients (Starovoitov, 1990; Gardner and Preston, 1992) of the iodine molecule, but none of them contributed to the determination or refinement of $\operatorname{Ar} \cdots \mathrm{I}_{2}$ PES.

The first pioneering theoretical studies (Beswick and Jortner, 1978b,a) on the Ar $\cdots \mathrm{I}_{2}$ Van der Waals complex used model pairwise Morse potentials very convenient for analytical studies (Table 1, entry 17). These and further works (Beswick and Jortner, 1980; Ewing, 1979, 1980, 1982; Halberstadt and Beswick, 1982; Ewing, 1986; Kokubo and Fujimura, 1986; Gray et al., 1986; Gray and Rice, 1986; Zhao and Rice, 1992; Buchachenko and Stepanov, 1993)) contributed a lot to the development of the theoretical formalism and the qualitative understanding of the VP dynamics, but not to the improvement of interaction PES.

The most realistic empirical $\mathrm{Ar}_{\cdots} \mathrm{I}_{2}(B)$ PES was suggested by Gray (1992) as a sum of pairwise Morse interactions (Table 1, entry 18) on the basis of experimental data by Blazy et al. (1980) and correlations with structural parameters of $\mathrm{Ar} \cdots \mathrm{Cl}_{2}$. This PES has been used in many thorough studies of $\operatorname{Ar}_{\cdots} \mathrm{I}_{2}(B)$ dynamics (Gray, 1992; Roncero et al., 1994b; Roncero and Gray, 1996; Roncero et al., 1996; Goldfield and Gray, 1997b; Bastida et al., 1997, 1999).

For completeness, a few words should be said about other empirical potentials implemented in the studies of large clusters and condensed phases. Most of such works (e.g. Hu and Martens, 1993a; Borrmann et al., 1993; Zadoyan et al., 1994a,b; Li et al., 1995; Ben-Nun et al., 1995; Liu et al., 1995; Liu and Guo, 1995; Schek et al., 1996) used simple Lennard-Jones pair potentials, common and convenient in molecular dynamics and Monte-Carlo simulations. In their study of the cage effect in $\operatorname{Ar}_{n} \cdots I_{2}$ clusters, Schröder 
and Gabriel (1996) derived a set of pairwise Morse potentials with a modified long range part. Like Gray's curves, they were parameterized mainly using the experimental data by Blazy et al. (1980).

\subsection{Diatomics-in-molecule (DIM) models}

DIM-based models, which provides a way for constructing the PES's of a polyatomic molecule from the electronic properties of its diatomic fragments (Tully, 1977; Kuntz, 1979, 1982), appear to be very popular and useful for studying the $\mathrm{Rg} \cdots \mathrm{X}_{2}$ complexes. Different approaches applied to these systems, primarily $\mathrm{He} \cdots \mathrm{Cl}_{2}$ and $\mathrm{Ar} \cdots \mathrm{I}_{2}$, provide not only a route for improving the accuracy of results, but also a qualitative insight in the importance of various factors governing their electronic structure. For completeness, we present in Appendix I a brief description of the particular DIM-based approach which covers and classifies all the models used so far for Ar $\cdots \mathrm{I}_{2}$.

In many cases, application of the DIM methodology to weakly-bound systems is hampered by the lack of reliable potentials for diatomic fragments. Fortunately, it is not the case for $\mathrm{Rg} \ldots \mathrm{X}_{2}$ complexes. Analysis of molecular-beam scattering data (Becker et al., 1979; Casavecchia et al., 1982; Aquilanti et al., 1988, 1990, 1993), ZEKE photoelectron spectroscopy of $\mathrm{Rg} \cdots \mathrm{X}^{-}$anions (Zhao et al., 1994; Yourshaw et al., 1996, 1998; Lenzer et al., 1998, 1999), and high-level ab initio calculations (Burcl et al., 1998; Lara-Castells et al., 2001; Buchachenko et al., 2001; Partridge et al., 2001) provided very accurate fragment interaction potentials. In the case of Ar...I, the most accurate potentials originate from ZEKE measurements (Zhao et al., 1994; Yourshaw et al., 1996). They are used in all DIM applications to Ar $\cdots \mathrm{I}_{2}$.

The first implementation of the DIM approach to a $\mathrm{Rg} \ldots \mathrm{X}_{2}$ system was made in 1993 
by Gersonde and Gabriel (1993) who investigated $\mathrm{Cl}_{2}$ photodissociation in solid Xe. They used the complete DIM method in the non-relativistic version $\left(\mathrm{I}_{2}\right.$ eigenstates were taken as pure Hund's case (a) $r$-independent functions, where $r$ stands for the $\mathrm{I}_{2}$ bond length), and the non-diagonal diabatic couplings between the $\mathrm{I}_{2}$ states of the same symmetry (two $2 \times 2$ blocks of ${ }^{1} \Sigma_{g}^{+}$and ${ }^{3} \Sigma_{u}^{+}$symmetry, see subsection 3.1$)$ were ignored.

Two years later, Naumkin and Knowles (1995) proposed a simple analytical formula to describe the ground-state interaction PES of $\mathrm{Rg} \cdots \mathrm{X}_{2}$ complexes:

$$
U_{X}=\sum_{\alpha=a, b}\left(V_{\Sigma}^{\alpha} \cos ^{2} \beta_{\alpha}+V_{\Pi}^{\alpha} \sin ^{2} \beta_{\alpha}\right)
$$

where, for brevity, $V_{\Lambda}^{\alpha}=V_{\Lambda}\left(\mathcal{R}_{\alpha}\right)$. This elegant formula corresponds to a diagonal element of the complete DIM Hamiltonian matrix of Gersonde and Gabriel (Buchachenko and

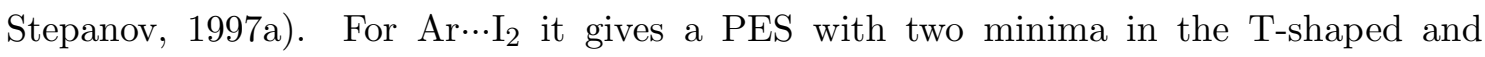
linear configuration with the well depths $D_{e}(T, X)=230 \mathrm{~cm}^{-1}$ and $D_{e}(L, X)=210 \mathrm{~cm}^{-1}$ (Table 1, entry 2). This model predicts a similar topology for the PES of other $\operatorname{Rg} \ldots \mathrm{X}_{2}(X)$ systems (Naumkin and McCourt, 1997, 1998a, 1999). It was proven to be very efficient in combination with $a b$ initio calculations when true $\mathrm{Rg}-\mathrm{X}$ interaction potentials are replaced by effective ones.

Soon after, another DIM-based model was suggested for Ar $\cdots \mathrm{I}_{2}$ (Buchachenko and Stepanov, 1996b) (its almost exact analog was independently developed and used for simulations of $\mathrm{I}_{2}$ photodynamics in condensed rare gases by Batista and Coker (1996)). The electronic wave functions of the bare $\mathrm{I}_{2}$ molecule (solutions of the Schrödinger equations for $\hat{H}_{0}$, see Eqs.(11) and (14) of Appendix I) were approximated by expansions over products of coupled Hund's case (c) $|j m\rangle$ atomic functions with coefficients (the $C_{k}^{n}(r)$ in Eq.(15) of Appendix I) frozen at their asymptotic $(r \rightarrow \infty)$ value. Because this approximation ne- 
glects a significant part of intramolecular interactions in the halogen molecule, it was called the intermolecular DIM, or IDIM, model. Its accuracy depends on the state considered. It is certainly valid for the $B\left({ }^{3} \Pi 0_{u}^{+}\right)$state which is the unique valence state in its symmetry representation. It is more questionable if there are several states of the same symmetry, as for the $X\left({ }^{1} \Sigma 0_{g}^{+}\right)$state, since the exact wave function should then be represented as an $r$-dependent linear combination of asymptotic solutions. For instance, the IDIM model gives a single T-shaped minimum with $D_{e}=249 \mathrm{~cm}^{-1}$ (Table 1, entry 19) for the $B$ state, in very good agreement with the experimental data (Blazy et al., 1980) and with the best empirical PES (Gray, 1992). The corresponding dissociation energy $D_{0}(B)=222 \mathrm{~cm}^{-1}$ falls within the error bars of the experimental estimation by Blazy et al. (1980). The corresponding PES for the ground state (Table 1, entry 3) also agrees well with Levy's data for $D_{0}(T, X)$, but it does not exhibit a minimum in the linear configuration.

The approximation which treats the $\mathrm{Ar}-\mathrm{I}_{2}$ interaction (the $\hat{H}_{1}$ term in Eq.(12) of Appendix I) as a first-order perturbation to the sum of monomer hamiltonians $\left(\hat{H}_{0}\right.$ in Eq.(11) of Appendix I) is called the IDIM PT1 approximation (Perturbation Theory 1st order). An attractive feature of this approximation is that all the electronic properties can be expressed in an analytical form (Buchachenko and Stepanov, 1997a, 1998a). In particular, the following simple formula for the $B$-state PES was derived:

$$
U_{B}=\frac{1}{4} \sum_{\alpha=a, b}\left[3 V_{\Sigma}^{\alpha}+V_{\Pi}^{\alpha}-\left(V_{\Sigma}^{\alpha}-V_{\Pi}^{\alpha}\right) \cos ^{2} \beta_{\alpha}\right] .
$$

This approach has been applied to several $\mathrm{Rg} \cdots \mathrm{X}_{2}(B)$ complexes, namely, $\mathrm{He} \cdots \mathrm{Cl}_{2}$ (Grig-

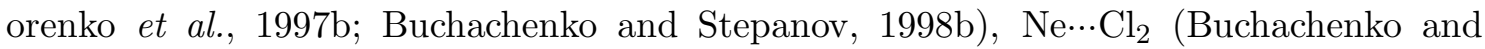

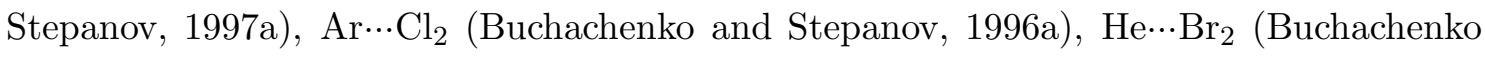
et al., 2000a; Hernández et al., 2000; Buchachenko et al., 2002), and in all cases very good agreement with experimental data on the $B\left({ }^{3} \Pi 0_{u}^{+}\right) \leftarrow X\left({ }^{1} \Sigma 0_{g}^{+}\right)$spectra and $B$ state 
VP dynamics has been obtained. Comparison between the IDIM and IDIM PT1 minima of the $B$ state presented in Table 1 indicates the validity of the first-order perturbative approximation.

In 1997, Grigorenko et al. published the results of a thorough DIM investigation of the $\mathrm{He} \cdots \mathrm{Cl}_{2}$ electronic structure (Grigorenko et al., 1997b), and showed that proper inclusion of the diabatic coupling matrix elements between the non-relativistic $\mathrm{X}_{2}$ electronic states of the same symmetry is necessary. For $\mathrm{Ar} \cdots \mathrm{I}_{2}$, a similar DIM approach was implemented by Naumkin (1998) but using Cartesian orbitals as atomic functions. With this choice, the diabatic couplings between the states of the same symmetry are implicitly included in a rather approximate manner (Pazyuk et al., 2001). As a result, the non-relativistic DIM method confirmed Naumkin-Knowles model [Eq.(3)], see Table 1, entry 5. It was also concluded that inclusion of SO coupling does not alter the ground state PES, but this finding may be subject to inaccuracy since the angular transformation from the $\mathcal{R}_{\alpha}$ to the $\mathbf{r}$ frame (see Appendix I) is applied only to the spatial part of the atomic basis functions, not to the spin one. The results for the $B$ state appeared to be similar to the IDIM PT1 data from Eq.(4) except for the existence of shallow linear minimum at long interfragment distances, cf. entries 19-21 in Table 1.

In the DIM studies reviewed above, the direct method for solving the $\mathrm{X}_{2}$ electronic structure problem was implemented. In other words, the $\hat{H}_{0}$ matrix was parameterized by the non-relativistic $\mathrm{X}_{2}$ curves taken from ab initio calculations. This gives rise to difficulties related to the diabatization of the $2 \times 2$ blocks of ${ }^{1} \Sigma_{g}^{+}$and ${ }^{3} \Sigma_{u}^{+}$symmetries due to the lack of $a b$ initio data. In addition, it also prevents the use of more accurate empirical information on the relativistic potential curves. To avoid these problems, the inverse method was applied by Pazyuk et al. (2001). The non-relativistic parameters of 
the $\hat{H}_{0}$ matrix, both energies and diabatic couplings, are adjusted to reproduce (in a least-square sense) the full set of the true relativistic energy curves of the molecule after diagonalization. The eigenvectors obtained are then used (Eqs. (15) and (16) of Appendix I) to construct analytical expressions for the PES and couplings, within the refined firstorder perturbation theory (DIM PT1) approach. The results give a global minimum in the T-shaped geometry and a secondary minimum in the linear configuration for the $X$ state PES, and the same, simple analytical formula of Eq.(4) for the $B$ state. Table 1 presents the minima of the PES obtained using two different sets of relativistic $\mathrm{I}_{2}$ curves, TP2 (entry 6, available empirical curves plus ab initio curves by Teichteil and Pélissier (1994)) and dJVN2 (entry 7, same as TP2 but ab initio curves by de Jong et al. (1997)), and Figure 2 presents the contour plots for the TP2 potential. The detailed analysis of the DIM PT1 results and comparison with other DIM models for $\operatorname{Ar}_{\cdots} \mathrm{I}_{2}(X)$ can be found in (Buchachenko et al., 2000b).

For completeness, one should also mention the very simple model Ar... $\mathrm{I}_{2}$ PES constructed from true Ar-I potentials (e.g. Fang and Martens, 1996; Conley et al., 1997; Meier et al., 1998). Although they do have some physical background, they are of course much less accurate than the PES available from the best DIM methods.

To conclude, the DIM approach provides a theoretically grounded and simple analytical interaction PES for the $B$ state, Eq.(4). The best results for the $X$ state PES obtained within the DIM PT1 model are in qualitative agreement with directly determined dissociation energies for both the linear (Stevens Miller et al., 1999) and the T-shaped (Blazy et al., 1980) isomer, with an error of the order of $30 \mathrm{~cm}^{-1}$. 


\subsection{Diabatic PES's and couplings for EP dynamics}

In order to understand the dynamics of the Ar $\cdots \mathrm{I}_{2}$ electronic predissociation, it is necessary to know the interaction PES for six crossing states (namely, $B$ " $1_{u}, 12_{g}, a^{\prime} 0_{g}^{+}, a 1_{g}$, $20_{u}^{-}$, and $3_{u}$, see subsection 3.1$)$ and their coupling with the $B\left({ }^{3} \Pi 0_{u}^{+}\right)$state, preferentially in the diabatic representation. The lack of direct experimental information and of a clear interpretation of the EP process strongly limited the possibility of an empirical approach. Under the commonly accepted assumption of a $B\left({ }^{3} \Pi 0_{u}^{+}\right)-a 1_{g}$ EP mechanism, a quite realistic approximation of the corresponding diabatic coupling was derived using the longrange multipole expansion of the electrostatic interaction (Roncero et al., 1996). However, this problem was first considered in its full complexity within the IDIM model. The topology of the PES for all crossing states was investigated. All of them have an attractive Ar- $\mathrm{I}_{2}$ interaction similar to those of the $X$ and $B$ states. Their couplings with the $B$ state were investigated by analyzing the contribution of the different states into the adiabatic $B$ state wave function (Buchachenko and Stepanov, 1996b) (the presentation of the corresponding results originally contained errors and is corrected in an Erratum (Buchachenko and Stepanov, 1997b)). The results were analyzed in terms of a simple symmetry model. In brief, treating the total electronic angular momentum as the orbital one, Hund's case (c) states of $\mathrm{I}_{2}$ can be approximately classified by irreducible representations of the $D_{\infty h}$ point group. Reduction of this group onto groups describing different configurations of the complex gives the symmetry correlations presented in Table 3. The states which are effectively coupled to the $B$ state according to the IDIM model are marked by an asterisk. Table 3 also indicates that in the T-shaped configuration of the complex EP can occur only through the $a 1_{g}$ state, whereas in the linear configuration it can only occur through the $a^{\prime} 0_{g}^{+}$state. The first finding supports the EP mechanism deduced from empirical 
approach (see section 6) and from a Golden-Rule wave packet treatment (Roncero et al., 1994b, 1996) which showed that the $a 1_{g}$ and not the $B^{\prime \prime} 1_{u}$ could be responsible for the EP process. However, it will be shown in section 6 that there are more states which can effectively predissociate the $B$ state for a given isomer than predicted from its equilibrium configuration, because of dynamical reasons.

In the frame of the IDIM PT1 model, analytical formulas for the diabatic PES and coupling matrix elements can be obtained (Buchachenko and Stepanov, 1998a; Buchachenko, 1998). The symmetry properties of the coupling functions are the same as in the complete IDIM treatment. The only exception is the $3_{u}$ state, for which the coupling to the $B$ state is predicted to be zero by IDIM PT1 and is found to be very weak (likely reflecting second-order interactions) in the IDIM approach.

The most accurate data on the PES and couplings for the crossing states have been obtained using DIM PT1 model from (Buchachenko et al., 2000b). They are briefly described in (Lepetit et al., 2002). Figure 3 shows the contour plots of the non-vanishing $B$ state couplings with the $B$ " $1_{u}, 12_{g}, a^{\prime} 0_{g}^{+}$, and $a 1_{g}$ states. The refined DIM PT1 approach does not alter the symmetry properties of the couplings and hence the important qualitative propensities to electronically predissociate through the $a 1_{g}$ and $a^{\prime} 0_{g}^{+}$states for the T-shaped and linear isomers, respectively.

\subsection{Ab initio calculations}

The first $a b$ initio calculations on the $\mathrm{Ar} \cdots \mathrm{I}_{2}(X)$ PES were performed in 1998 by Kunz et al. (1998) who used a variety of extended atomic orbital (AO) basis sets and the methods of correlation treatment. Studying the electronic structure of the Ar...I and $\mathrm{I}_{2}$ fragments, these authors concluded that within an all-electron treatment the SO interaction 
should not be essential for the interaction PES. Their best results were obtained at the non-relativistic coupled cluster $\operatorname{CCSD}(\mathrm{T})$ (coupled cluster expansion including single and double excitations with non-iterative correction to triple excitations) level of theory. They are presented in Table 1 (entry 10) together with less accurate data by second and fourth order Møller-Plesset perturbation theory (MP2 and MP4, entries 8 and 9). The results vary depending on the method, but the essential features of the PES - a global minimum at the linear geometry and a secondary minimum at the T-shaped configuration — remain unaltered.

Almost simultaneously and independently Naumkin (1998) carried out a very similar $a b$ initio study (CCSD-T) and obtained results in a qualitative agreement with those of Kuntz et al., with a difference of a few tens of wave numbers due to a smaller basis set (see Table 1, entry 11). For the same reason, the calculated binding energies of the ${ }^{2} \Sigma^{+}$ and ${ }^{2} \Pi$ states of the Ar..I fragment underestimate the measured ones (Zhao et al., 1994) by 100 and $50 \mathrm{~cm}^{-1}$, respectively. Using Eq.(3) and introducing various corrections to the $a b$ initio potentials, Naumkin and McCourt constructed a family of improved PES, one of them giving the dissociation energies $D_{0}(T, X)=233 \mathrm{~cm}^{-1}$ and $D_{0}(L, X)=237 \mathrm{~cm}^{-1}$. These results were considered as an argument in favor of Klemperer's energetics of the $\operatorname{Ar} \cdots \mathrm{I}_{2}(X)$ complex.

However, in 2001 Naumkin reported an improved ab initio study (Naumkin, 2001). It used the same CCSD-T methodology with an extended basis set, and incorporated relativistic effective core potential (RECP) for the inner shells of the iodine atoms. As a result, significantly deeper minima were obtained (Table 1, entry 12), but the Ar-I interaction remained underestimated by ca. $35 \mathrm{~cm}^{-1}$. With the help of Naumkin-Knowles model (3), the "best ab initio estimations" for $D_{0}(T, X)$ and $D_{0}(L, X)$ were obtained as 
$242 \pm 11 \mathrm{~cm}^{-1}$ and $250 \pm 8 \mathrm{~cm}^{-1}$, respectively.

The most recent and accurate calculations on the $\operatorname{Ar} \cdots \mathrm{I}_{2}(X)$ PES have been reported by Prosmiti et al. (2002b). Although they used practically the same method as Naumkin, a remarkable improvement was achieved by augmenting the atomic orbital basis set by bond functions which provide an efficient way of saturating the basis set for a correct treatment of dispersion interaction (Chałasiński and Szczȩśniak, 1994). The resulting binding energies of both isomers are larger (Table 1. entry 13). Calculation of the zeropoint energy yielded $D_{0}(T, X)=212 \mathrm{~cm}^{-1}$ and $D_{0}(L, X)=237 \mathrm{~cm}^{-1}$.

To summarize, ab initio calculations tend to converge the binding energy of the Tshaped isomer to the value conforming Levy's data. They always predict the linear isomer to be lower in energy, in agreement with Burke and Klemperer's hypothesis (Burke and Klemperer, 1993b), but the dissociation energy seems to converge to a larger value than the one obtained by Stevens Miller et al. (Stevens Miller et al., 1999), and to a smaller energy difference with the perpendicular isomer than deduced from intensity ratios in the experiment of Burke and Klemperer.

If the ultimate goal of electronic structure theory is to provide accurate electronic characteristics for the quantitative determination of spectroscopic and dynamical observables, theoretical investigations of $\mathrm{Ar} \cdots \mathrm{I}_{2}$ are far from being finished. Ab initio and DIM methodologies do not completely agree with each other and with directly determined experimental energies. The dissociation energy of the linear isomer is still uncertain. There is only one experimental result (Stevens Miller et al., 1999), which agrees fairly well with one ab initio result (Kunz et al., 1998) and DIM calculations (Buchachenko et al., 2000b), but not with more recent and better converged ab initio results (Naumkin, 2001; Prosmiti et al., 2002b). The dissociation energy of the perpendicular isomer seems to be better 
established since there are two independent but identical experimental estimates (Blazy et al., 1980; Burroughs and Heaven, 2001), supported by recent DIM (Buchachenko et al., 2000b) and ab initio (Naumkin, 2001; Prosmiti et al., 2002b) results. The contradicting experimental estimate (Stevens Miller et al., 1999) is an indirect one, subject to questions and uncertainties.

\section{The one-atom cage effect}

Saenger et al. (1981) showed evidence for recombination of $\mathrm{I}_{2}$ excited above the $B$ state dissociation limit when $\mathrm{I}_{2}$ was complexed with one or more atoms or molecules, whereas uncomplexed $\mathrm{I}_{2}$ exhibits 100\% dissociation (Burde et al., 1974). Valentini and Cross (1982) reported the observation of the "one-atom cage effect" by recording the dispersed fluorescence of recombined $\mathrm{I}_{2}(B)$ produced upon excitation of the Ar $\cdots \mathrm{I}_{2}$ complex at $488 \mathrm{~nm}$, $448 \mathrm{~cm}^{-1}$ above the $B$ state dissociation limit of $\mathrm{I}_{2}$. Their results showed that the cage effect produces $\mathrm{I}_{2}$ in vibrational levels $\left(23 \leq v^{\prime} \leq 49\right)$ that lie from $800 \mathrm{~cm}^{-1}$ to more than $2300 \mathrm{~cm}^{-1}$ below the initially excited $\mathrm{I}_{2}$ energy. Such a large energy transfer, much larger than the one observed in vibrational predissociation of Ar $\cdots \mathrm{I}_{2}$ (Johnson et al., 1981), was interpreted as a purely kinematic (ballistic) mechanism, namely, impulsive transfer from $\mathrm{I}_{2}$ to Ar which dissociates the complex. A three-dimensional quasi-classical study by Noorbatcha, Raff, and Thompson (1984), using an empirical pairwise potential with parameters taken from Beswick and Jortner (1978b), indicated that efficient impulsive energy transfer could occur from near collinear geometries, but the amount of energy transfer was not as large as the one measured by Valentini and Cross (1982). An "anchoring" effect due to the attraction exerted by the argon atom on the departing I atoms from near T-shape initial geometries could result in long-lived, complex trajectories also leading to stabilization of 
$\mathrm{I}_{2}$, but it is a much slower and less efficient energy transfer process. Subsequent, extensive dispersed fluorescence experiments conducted by Philippoz, Van den Bergh, and Monot (Philippoz et al., 1986, 1987, 1990) on $\mathrm{Rg} \cdots \mathrm{I}_{2}$ complexes gave a more complete picture of the one-atom cage effect. They reported product vibrational state distributions obtained at several photodissociation wavelengths $(496.5,488$, and $476.5 \mathrm{~nm}$, corresponding to 98 , 448, and $943 \mathrm{~cm}^{-1}$ above the dissociation limit, respectively) for $\mathrm{Ne} \cdots \mathrm{I}_{2}, \mathrm{Ar} \cdots \mathrm{I}_{2}, \mathrm{Kr} \cdots \mathrm{I}_{2}$, and $\mathrm{Xe} \cdots \mathrm{I}_{2}$. The most probable recoil energy increased with increasing excitation energy and with increasing mass. For Ar $\cdots \mathrm{I}_{2}$, it was around 815,965 , and $1165 \mathrm{~cm}^{-1}$ for $\lambda=$ 496.5, 488, and 476.5 nm excitation, respectively, which was significantly larger than the prediction by Noorbatcha et al..

At the time at which these results were obtained, there was no definitive structural information available for the Ar... $\mathrm{I}_{2}$ complex, see preceeding section. However, in analogy to $\mathrm{He} \cdots \mathrm{I}_{2}$, the common belief was that the $\mathrm{Ar} \cdots \mathrm{I}_{2}(X)$ complex had a T-shaped structure. For this structure, a purely kinematic energy transfer cannot be very efficient, which made the one-atom cage effect stand as somewhat of an enigma.

An alternative mechanism for the cage effect was then proposed by Beswick et al. (1987), involving two electronic states. In this mechanism, initial continuum excitation is not to the $B\left({ }^{3} \Pi 0_{u}^{+}\right)$state, but to the repulsive $B^{\prime \prime} 1_{u}$ state (Fig. 1 ), which contributes significantly to the photon absorption cross section in the $\lambda=500-450 \mathrm{~nm}$ wavelength region (Tellinghuisen, 1982) (the strength of the $B^{\prime \prime} 1_{u} \leftarrow X\left({ }^{1} \Sigma 0_{g}^{+}\right)$absorption is about 0.6 that of the $B\left({ }^{3} \Pi 0_{u}^{+}\right) \leftarrow X\left({ }^{1} \Sigma 0_{g}^{+}\right)$one at $\left.488 \mathrm{~nm}\right)$. The $B^{\prime \prime}$ and $B$ states are weakly coupled by magnetic and hyperfine interactions in the free $\mathrm{I}_{2}$ molecule (Broyer et al., 1975, 1976). The presence of a solvent atom or molecule can induce a stronger coupling between these two states, see section 3.5. The proposed mechanism, usually called "non-adiabatic 
cage effect", was then

$$
\operatorname{Ar} \cdots \mathrm{I}_{2} \stackrel{h \nu}{\longrightarrow} \operatorname{Ar} \cdots \mathrm{I}_{2}\left(B^{\prime \prime}, E\right) \stackrel{C}{\longrightarrow} \operatorname{Ar}+\mathrm{I}_{2}(B, v) .
$$

Assuming that the electronic nonadiabatic coupling $C$ is a slowly varying function of the $\mathrm{I}_{2}$ internuclear coordinate, the rates for $\mathrm{I}_{2}$ recombination to the final vibrational levels $v$ of the $B$ state are proportional to the Franck Condon factors

$$
k_{\left(B^{\prime \prime}, E\right) \rightarrow\left(B, v^{\prime}\right)} \propto\left|\left\langle\chi_{E}^{B^{\prime \prime}} \mid \chi_{v}^{B}\right\rangle\right|^{2}
$$

where $\chi_{E}^{B^{\prime \prime}}$ is the continuum function for the $\mathrm{I}-\mathrm{I}$ motion in the $B^{\prime \prime}$ dissociative state at energy $E=E_{0}+h \nu$ and $\chi_{v}^{B}$ a final vibrational wave function in the $B$ state. This model gave final distributions of $\mathrm{I}_{2}(B, v)$ states (Beswick et al., 1987; Roncero et al., 1994a) very similar to the experimental results of Philippoz et al. (1987).

In order to distinguish between the purely kinematic and the nonadiabatic models for the cage effect, Burke and Klemperer (Burke and Klemperer, 1993b) studied the absorption and fluorescence of $\mathrm{Ar} \cdots \mathrm{I}_{2}$ in the bound region of the $B$ state. In that region the $B\left({ }^{3} \Pi 0_{u}^{+}\right) \leftarrow X\left({ }^{1} \Sigma 0_{g}^{+}\right)$transition intensity is localized in discrete bands, while the $B^{\prime \prime} 1_{u} \leftarrow X\left({ }^{1} \Sigma 0_{g}^{+}\right)$transition remains a continuum. If the nonadiabatic model was true, exciting between the lines of the $B \leftarrow X$ transition (hence exciting the $B^{\prime \prime}$ continuum) would lead to fluorescence from $B$ state $\mathrm{I}_{2}$, whereas a purely kinematic mechanism would yield dissociation and no fluorescence. The experimental results showed the existence of fluorescence from continuum excitation in the region $v_{B}^{\prime} \geq 14$, with a wavelength dependence of the relative intensity that was adequately modeled by the mechanism proposed by Beswick et al. (1987), but the measured absolute intensity of the fluorescence was much too large to be due to the sole excitation of the $B^{\prime \prime} 1_{u}$ state. The total continuum intensity integrated over the range of the $\mathrm{I}_{2}\left(B, v_{B}^{\prime}=26\right) \leftarrow\left(X, v^{\prime \prime}=0\right)$ vibronic band was deter- 
mined to be $2.1 \pm 0.4$ times the integrated intensity of the corresponding discrete band of Ar $\cdots \mathrm{I}_{2}$. However, at this excitation wavelength, the transition intensity to the $B^{\prime \prime}$ state is only 0.13 of that to the $B$ state (Tellinghuisen, 1982).

Burke and Klemperer proposed that this continuum could be due to the existence of a linear isomer. Brown, Schwenke, and Truhlar (1985) had reported comparable minima for linear and T-shaped geometries in a calculated potential energy surface for $\mathrm{He}_{\cdots} \cdots \mathrm{I}_{2}$, but there was no $a b$ initio study for Ar $\cdots \mathrm{I}_{2}$ at the time. In contrast to the T-shaped isomer, there could be a large difference in the equilibrium bond length and binding energy of the $B$ state relative to the $X$ state. It was argued that the zero occupancy of the $\sigma^{*}$ orbital in the $X$ state may result in a fairly short equilibrium bond length for the linear isomer. Upon excitation to the $B$ state, a $\pi^{*}$ electron is transferred to the $\sigma^{*}$ orbital, thus increasing this bond length and lowering the binding energy. This can result in a continuum absorption spectrum for this isomer if the difference in bond lengths are large enough. The linear isomer could then account for the one-atom cage effect observed above the $B$ state dissociation limit by a purely kinematic effect.

A three-dimensional quasiclassical trajectory study by Miranda et al. (1994) concluded that the one-atom cage effect could not be due to a linear isomer, using Gray's pairwise interaction potential (Gray, 1992), see section 3. Caging was indeed observed, and energy transfer was more important for the linear than for the T-shaped structure, but it was still too low compared to experiments. However, the $X$ state configuration of the linear isomer was only guessed, in the absence of any information. In particular, the Ar-I bond length was taken to be the same one as the Ar-I distance in the perpendicular configuration, which revealed to be wrong in later DIM and ab initio studies. In a classical simulation of the $\mathrm{I}_{2} \cdots \mathrm{Rg} g_{n}$ cage effect using empirical potentials (see end of section 3.3), Schröder 
and Gabriel (1996) concluded that in order to explain the cage effect, the Van der Waals binding energy had to be increased, or more than one rare-gas atom had to be bound to $\mathrm{I}_{2}$, or the configuration of the one-atom complex had to be collinear with a larger $\mathrm{I}_{2}-\mathrm{Rg}$ equilibrium distance in the $B$ state compared to the $X$ state.

In a wave packet calculation restricted to the collinear configuration, Fang and Martens (1996) showed that by using model interaction PES deduced from the known $\mathrm{I}\left({ }^{2} P_{3 / 2}\right)-\mathrm{Ar}$ and $I^{*}\left({ }^{2} P_{1 / 2}\right)$-Ar interactions (see section 3.4 ), good agreement with experiment was obtained. However, it was not clear if this result would still be valid using the experimentally validated $\mathrm{Ar}-\mathrm{I}_{2}(B)$ potential and getting out of the purely collinear configuration (which has strictly speaking a probability of zero because of the solid angle volume element). In a three-dimensional wave packet study using the ab initio PES by Kunz et al. (1998) for the $X$ state and the IDIM PT1 PES for the $B$ state, Zamith et al. (1999) confirmed the possibility of a purely kinematic origin of the one-atom cage effect from the linear isomer of $\mathrm{Ar} \cdots \mathrm{I}_{2}$, and showed that the vibrational distributions depended strongly on the ground and excited state equilibrium geometries. A very good agreement with experimental final vibrational distributions was obtained by increasing the equilibrium distance in the I-Ar interaction potentials in IDIM PT1 PES by $2 \%$ (Fig. 4).

Additional experimental indications that the kinematic mechanism of direct, impulsive energy transfer in the collinear isomer is responsible for the one-atom cage effect have been obtained. The existence of two geometrically distinct forms has been demonstrated in the photoionization study of Cockett et al. (Cockett et al., 1996). Burroughs, Van Marter, and Heaven (Burroughs et al., 1999) have reported the results of fluorescence-depletion, i.e. "hole-burning", experiments which demonstrate that fluorescence from free $\mathrm{I}_{2}$ produced by excitation above the $B$ state dissociation limit is not depleted by excitation to the T- 
shaped $B \leftarrow X$ band of Ar $\cdots \mathrm{I}_{2}$. Fluorescence depletion was indeed observed by excitation to the adjacent continuum which had been assigned to the linear isomer by Burke and Klemperer (1993b). This shows that, even though direct absorption to the $B^{\prime \prime} 1_{u}$ state exists, nonadiabatic coupling to the $B$ state due to the presence of the argon atom is not strong enough to produce any appreciable amount of caging from the perpendicular isomer. The one-atom cage effect is thus due to the collision of the dissociating I atom with the argon atom near the linear configuration. The same effect was surmised by Wan et al. (1997) to explain their experimental results on caging of $\mathrm{I}_{2}$ by collisions with rare gas atoms at room temperature. It was confirmed in a wave packet simulation by Meier, Engel and Beswick (1998), who obtained very good agreement with the recurrences observed in the pump-probe signal. They observed that effective collisional caging can only occur if the collision leads to a large momentum transfer from the iodine to the Ar-atom, which is the case if the collision occurs at small angles, i.e. close to the collinear case, and at small I-I distances, where (due to the well of the $B$ state potential) the relative motion of the dissociating I atoms is fast. This is why it is important for caging in the Van der Waals cluster that the intermolecular bond length in the $X$ state be shorter than in the $B$ state: vertical excitation brings $\mathrm{Ar} \cdots \mathrm{I}_{2}$ close to the hard sphere collision distance in the $B$ state, so that one of the departing I atoms hits the argon with a high velocity, therefore transferring a large amount of momentum.

Caging of $\mathrm{I}_{2}$ was also observed in large rare gas clusters (Liu et al., 1993) and matrices (Beeken et al., 1983; Macler and Heaven, 1991; Zadoyan et al., 1994a,b; Benderskii et al., 1997). Many of the matrix studies were done in the bound state region of the $B$ state, where the process is more complex. It first implies electronic predissociation of the $B$ state, followed by caging, or initial excitation to the dissociative $B^{\prime \prime}$ or the repulsive region of the 
weakly bound $A 1_{u}$ state. Fluorescence from $B$ state recombined $\mathrm{I}_{2}$ was indeed observed. In addition, infrared emission was also detected from the $A 1_{u}$ and $A^{\prime} 2_{u}$ states which are weakly bound states going to the $\mathrm{I}\left({ }^{2} P_{3 / 2}\right)+\mathrm{I}\left({ }^{2} P_{3 / 2}\right)$ dissociation limit. Batista and Cocker (1997) conducted a nonadiabatic molecular dynamics simulation of a time-resolved pumpprobe experiment of $A$ and $B$ state $\mathrm{I}_{2}$ in a rare gas matrix, similar to the experiments by Apkarian and coworkers (Zadoyan et al., 1996). In the region of the $B$ state excitation, they do not consider the possible excitation to the $A$ or $B^{\prime \prime} 1_{u}$ states which also contribute to the absorption cross section. However, the dynamics following excitation to the $B$ state is rich and complex. The excited molecules can either remain in the $B$, predissociate to one of the three $B^{\prime \prime} 1_{u}, 12_{g}$, or $a 1_{g}$ states during their early time dynamics. The predissociated molecules recombine after hitting the cage atoms into the $A 1_{u}, A^{\prime} 2_{u}$, and $X$ states. It can be noted that $\mathrm{I}_{2}$ occupies initially a double substitution site in an undistorted fcc argon crystal, which puts it in a collinear configuration with some of its nearest neighbors. This is clearly a different situation from exciting $\mathrm{I}_{2}$ above its $B$-state dissociation limit where dissociation is direct. However, it does show that nonadiabatic effects can be quite important in the condensed phase. One important difference with the one-atom cage effect is that the argon atoms do not evaporate, so that when the I-I distance is very long and the I atoms collide with the argon atoms of the cage, they feel a strong I-Ar interaction which couples the $\mathrm{I}_{2}$ states.

Solvent-induced dissociation and caging dynamics of $\mathrm{I}_{2}(B)$ was also studied by a timeresolved pump-probe experiment in supercritical rare gas solvents (Lienau and Zewail, 1996) and the role of these $A$ and $A^{\prime}$ states was also put in evidence. It would be interesting to look for the possibility of observing these states in the one-atom cage effect. 


\section{Vibrational Predissociation, Intramolecular Vibrational Relaxation and spectra}

\subsection{Experimental data for the T-shaped isomer VP dynamics}

When the $\mathrm{Rg} \ldots \mathrm{I}_{2}$ Van der Waals molecules are excited in the bound spectral region of $\mathrm{I}_{2}(B)$, the fluorescence excitation spectra show broadened features associated to $\operatorname{Rg} \cdots \mathrm{I}_{2}\left(B, v^{\prime}\right)$ quasi-bound levels which decay into a dissociative continuum by predissociation.

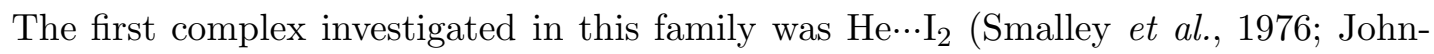
son et al., 1978; Sharfin et al., 1979), reviewed in (Levy, 1981). Levy and co-workers determined the broadening of the lines as a function of $v^{\prime}$, the vibrational excitation of $\mathrm{I}_{2}(B)$ within the complex, by detecting the fluorescence of the $\mathrm{I}_{2}\left(B, v<v^{\prime}\right)$ products. The width of the peaks showed a monotonic increase as a function of $v^{\prime}$, the $v=v^{\prime}-1$ vibrational level of $\mathrm{I}_{2}$ being the dominant final state, by more than $90 \%$. Soon after the first measurements, Beswick and Jortner (1977; 1978b; 1978a; 1981), Ewing (1979), and others (J.A. Beswick, G. Delgado-Barrio and J. Jortner, 1979; Beswick and Jortner, 1980; Delgado-Barrio et al., 1983), interpreted these results in terms of the energy or momentum gap law: the coupling between the $\mathrm{He} \cdots \mathrm{I}_{2}\left(B, v^{\prime}\right)$ quasi-bound state and the $\mathrm{He}+$ $\mathrm{I}_{2}\left(B, v^{\prime}-1\right)$ dissociative continuum grows larger as the final kinetic energy between the fragments decreases.

However, at higher excitation energies, when the $\Delta v=-1$ channel closes, the monotonous increase of the measured linewidths with $v^{\prime}$ suddenly stops, and thereafter behaves erratically with $v^{\prime}$. This behavior has been observed for complexes like $\mathrm{He} \cdots \mathrm{I}_{2}, \mathrm{Ne} \cdots \mathrm{I}_{2}, \mathrm{He} \cdots \mathrm{Br}_{2}$ (L. J. van der Burgt and Heaven, 1984; Jahn et al., 1994, 1996), Ne..Br ${ }_{2}$ (Cline et al., 
1987), or $\mathrm{Ar} \cdots \mathrm{Cl}_{2}$ (Evard et al., 1988a). It is due to the presence of secondary quasibound states associated to the closed $v^{\prime}-1$ channels (Roncero et al., 1988; Halberstadt et al., 1992a,b; González-Lezana et al., 1996). Dissociation now occurs predominantly in a stepwise fashion : $\operatorname{Rg} \cdots \mathrm{X}_{2}\left(n^{\prime}, v^{\prime}\right) \rightarrow \operatorname{Rg} \cdots \mathrm{X}_{2}\left(n^{\prime \prime}>n^{\prime}, v^{\prime}-1\right) \rightarrow \operatorname{Rg}+\mathrm{X}_{2}\left(v=v^{\prime}-2\right)$. This mechanism corresponds to the Intramolecular Vibrational Relaxation (IVR) process, where vibrational quanta are transferred in a sequential fashion from the stretching mode of the I-I molecule to Van der Waals modes (with an excitation defined by a collective quantum number $n$ ) until this weak bond breaks. Because the energy difference between the interacting initial "bright" and intermediate "dark" quasi-bound states changes as a function of $v^{\prime}$, the rate of dissociation depends strongly on $v^{\prime}$ in a very oscillatory way. The erratic dependance of the VP rate as a function of initial excitation is a fingerprint that IVR is in the sparse regime (the different IVR regimes are presented in Appendix II). Sparse IVR has a second observable fingerprint on rotational distributions, as shown in $\mathrm{Ar} \cdots \mathrm{Cl}_{2}$ (Evard et al., 1988a) and $\mathrm{He} \cdots \mathrm{Br}_{2}$ (Rohrbacher et al., 1999a). Since the "dark" state acts as a doorway for dissociation, the final rotational distribution of the halogen fragments depends strongly on the nature of the "dark" state, especially on its bending character. As a result, complicated oscillatory rotational distributions which depend strongly on the initial excitation are obtained in this regime. In addition, due to the $\mathrm{I}_{2}$ anharmonicity, the relative energies of bright and dark states change with $v^{\prime}$ and so does the bending character of the doorway state. Thus, rotational distributions are strongly dependent on the initial excitation.

The dynamics of Ar $\cdots \mathrm{I}_{2}$ complexes presents special features as compared to the lighter complexes of the same family. One is the balanced competition between electronic and vibrational predissociation, which is analyzed in section 6 . Another is related to the final 
vibrational state distribution of the $\mathrm{I}_{2}(B, v)$ products after excitation of $\operatorname{Ar} \cdots \mathrm{I}_{2}\left(B, v^{\prime}\right)$, as measured by Levy and co-workers (Johnson et al., 1981) : they found that the first open channel is $v^{\prime}-3$. This results from the value of the binding energy of the $\operatorname{Ar} \cdots \mathrm{I}_{2}(\mathrm{~B})$ complex, which is believed to be larger than two vibrational quanta of the uncomplexed $\mathrm{I}_{2}(B)$ molecule in the energy region of interest (see section 3). The stepwise mechanism now involves two sets of intermediate "dark" states $\mathrm{Ar} \cdots \mathrm{I}_{2}\left(n^{\prime \prime}>n^{\prime}, v^{\prime}-1\right)$ and $\mathrm{Ar} \cdots \mathrm{I}_{2}\left(n^{\prime \prime \prime}>\right.$ $\left.n^{\prime \prime}, v^{\prime}-2\right)$. Due to this increase of intermediate level density, one may wonder whether IVR still occurs in the sparse regime or approaches intermediate or even statistical regimes (see Appendix II). This question is still nowadays open. On the one hand, real-time picosecond measurements have shown that the kinetics of formation of the $I_{2}$ product follow a simple exponential law (Breen et al., 1990; Willberg et al., 1992). Also, the decay rates appear not to be significantly modified by initial Van der Waals excitation (Burke and Klemperer, 1993a). These facts advocate for a dense regime IVR. Assuming such a regime, VP rate is expected to increase monotonously with $v^{\prime}$, and EP would be responsible for the oscillations in the fluorescence intensity, as will be discussed later in section 6. However, experimental product rotational distributions (Burroughs and Heaven, 2001) show structures which are reminiscent of sparse regime IVR, an idea which is also supported by all recent quantum calculations on this system. Addressing the problem of the IVR regime in VP dynamics should also help in understanding the origin of the oscillations in the fluorescence intensity.

\subsection{Theoretical interpretations for the T-shaped isomer VP dynamics}

As in the $\mathrm{He} \cdots \mathrm{I}_{2}$ case, the first theoretical modeling of $\mathrm{Ar} \cdots \mathrm{I}_{2}$ assumed a direct mechanism in the collinear geometry within a Distorted Wave approximation (Beswick and Jortner, 1980) and found a monotonous increase of the VP rate with increasing $v^{\prime}$. In addition, 
the VP rates were in rather good agreement with those obtained in real-time experiments (Breen et al., 1990; Willberg et al., 1992). This apparent good agreement between theory and experiment fails down when it is considered, as noted by Burke and Klemperer (1993a), that the transitions studied correspond to a T-shaped complex.

The first quantum three-dimensional calculations on $\mathrm{Ar} \cdots \mathrm{I}_{2}$ were performed by Gray (1992) using a time-dependent wavepacket method and accurate empirical pairwise PES. He found that the population decrease of the initial state does not follow an exponential law, as it is the case for the direct mechanism, but presents oscillations attributed to the presence of several resonances with energies and widths obtained by de Prony's method. Thus, using the dominant resonance width, Gray found that the ratio of linewidths $\Gamma_{v^{\prime}=21} / \Gamma_{v^{\prime}=18}$ was consistent with the experimental one obtained from the total rates measured by Zewail and co-workers (Breen et al., 1990; Willberg et al., 1992) and the vibrational predissociation efficiencies obtained by Goldstein et al. (1986).

The influence of IVR on the VP dynamics of Ar $\cdots \mathrm{I}_{2}$ was later analyzed (Gray and Roncero, 1995; Roncero and Gray, 1996) using time-dependent as well as time-independent calculations on the same PES. These essentially exact calculations (from the dynamical point of view) were nicely reproduced by approximate analytical models based on standard radiationless transition treatments (Roncero and Gray, 1996), extending works already performed on $\mathrm{Ar} \cdot \mathrm{Cl}_{2}$ (Halberstadt et al., 1992a,b). It was possible to characterize in details the number and the nature of the zero-order bound states involved. As an example, the absorption spectrum and vibrational population $v s$ time are shown in Figs. 5 and 6 , respectively, for the vibrational predissociation of $\operatorname{Ar}_{\cdots} \mathrm{I}_{2}\left(B, v^{\prime}=21\right)$. The analytical model requires only a few bound states, three in this particular case, belonging to the $v^{\prime}$ (bright state), $v^{\prime}-1$ and $v^{\prime}-2$ (dark states) manifolds. As already noted, three vibrational 
quanta are required to fragment the $\mathrm{Ar} \cdots \mathrm{I}_{2}$ complex, and it can be clearly considered as a sequential mechanism as was the case in $\mathrm{Ar} \cdots \mathrm{Cl}_{2}$. The population, initially in the $v^{\prime}$ channel, is transfered to $v^{\prime}-1$. Once the population in the $v^{\prime}-1$ channel becomes significant, population starts building up in channel $v^{\prime}-2$, and dissociation starts in channel $v^{\prime}-3$ once there is enough population in $v^{\prime}-2$. Because of this sequential mechanism, the dissociation probability shows a clear non-exponential behavior (Roncero et al., 1993, 1997). The exact picture is a bit more complicated, though. At each vibrational step, the population bifurcates to go not only to the $v-1$ manifold, but also to the $v+1$ one. In addition, there are contributions from other energy pathways involving steps with the transfer of more than one quantum. As a conclusion, it was found that the VP of Ar $\cdots \mathrm{I}_{2}$ is mediated by IVR, involving only a few zero-order bound states (sparse limit). Because the coupling between those few bound states depends on their mutual separation, it was found (Gray and Roncero, 1995; Roncero and Gray, 1996) that the VP rate presents oscillations as a function of $v^{\prime}$.

How can these oscillations be conciliated with the experimental assumption of a monotonous dependence of the VP rate, made by Burke and Klemperer (1993a) in order to interpret the relative efficiencies of the VP and EP processes (see section 6 for details)? This monotonous increase is the fingerprint of IVR in the statistical limit, where the "bright" state always "faces" a second "dark" state due to their relatively high density. The possibilities for explaining the discrepancies on the dependence of the VP rate with $v^{\prime}$ are the following:

i) The potential is inadequate to describe VP. However, several parametrizations were used (Roncero and Gray, 1996) and the sparse limit IVR was obtained in all calculations;

ii) The widths of the dark states may be increased by coupling to the continuum 
through EP, thus changing the IVR regime (see section 6); and

iii) For such heavy systems, the total angular momentum can be quite large and the density of states is expected to increase. Also, rotational averaging corresponding to the experimental conditions may smooth out VP rate oscillations.

Let us consider in more details the effect of the total angular momentum on the VP dynamics. Some early time-independent calculations on $\mathrm{Ar} \cdots \mathrm{Cl}_{2}$ showed an extreme sensitivity not only to the value of $J$ but also to its projection on a body-fixed frame axis (Roncero et al., 1993). The same situation occurred for Ar $\cdots \mathrm{I}_{2}$ for low angular momentum. Time-dependent and time-independent calculations showed the sensitivity due to the change in the relative energies of the "dark" states and "bright" ones as a function of $J$ (indeed, dark states have smaller rotational constants than bright ones, being more excited in the Van der Waals mode (Roncero et al., 1993)).

Following this line, time-dependent calculations for high $J$ values were performed on Ar $\cdots \mathrm{I}_{2}$, by Goldfield and Gray $(1997 \mathrm{~b} ; 1997 \mathrm{a})$, and on $\mathrm{Ar} \cdot \mathrm{Cl}_{2}$ by Roncero et al. (1997). When varying $J$ values up to 15 or 20 , the IVR regime remained in the sparse-intermediate regime if the initial rotational sublevel $K$ (where $K$ denotes the $(2 J+1)$ sublevels by increasing energy order) is small and unchanged. However, when both $J$ and $K$ are modified, the effect is larger (Roncero et al., 1997). A good example is provided by $\mathrm{Ar} \cdots \mathrm{Cl}_{2}\left(B, v^{\prime}=18\right)$, where three vibrational quanta are required for fragmenting the complex as in $\mathrm{Ar}_{\cdots} \mathrm{I}_{2}$ (see Fig. 7). In this case for $J=0$ and for $(J=15, K=1)$ the autocorrelation functions show clear recurrences attributed to sparse-intermediate regimes, while for $(J=15, K=2 J)$ the recurrences tend to disappear, clearly showing a tendency towards the statistical limit. This was shown to reflect the character of the initial bright state due to Coriolis coupling on the bound states rather than the effect of the Coriolis 
coupling on the dynamics which is rather weak. The bright state $K=0$ is mainly of $\Omega=0$ character, where $\Omega$ stands for the projection of the total angular momentum on the axis which connects Ar to the center of mass of the dihalogen. Bright states corresponding to large $K$ have components on a larger range of $\Omega$ values. As a result, more dark states are involved in the dissociation dynamics of a large $K$ bright state than of a low $K$ one. However, another calculation on Ar $\cdots \mathrm{I}_{2}$ (Goldfield and Gray, 1997b,a) was performed for $J=10$, and sparse limit IVR was obtained. Therefore, more complete calculations, including higher angular momenta, proper average over the initial thermal rotational distribution and taking into account EP channels, would be most useful to elucidate the role of the total angular momentum in $\mathrm{Ar} \cdots \mathrm{I}_{2}$.

Another way to modify the IVR regime is to increase the initial vibrational excitation of the diatomic subunit. One may expect that the increase of dark state density induced by the anharmonicity will change the IVR regime from a sparse to a dense one. This has been observed in $\mathrm{Ar} \cdots \mathrm{Cl}_{2}$ (Roncero et al., 1997) or $\mathrm{Ne} \cdots \mathrm{Br}_{2}$ (Roncero et al., 2001a): the IVR bands associated to increasing $v^{\prime}$ values get more and more congested. However, close to the dissociation limit, some narrow peaks suddenly appear again, associated to resonances where the rare gas atom is inserted between the two halogen atoms (Roncero et al., 2001a; Prosmiti et al., 2002c). The halogen diatomic is so stretched that VP becomes inefficient, thus introducing some sparse character in the spectra. It would be interesting to perform a similar study on $\mathrm{Ar} \cdots \mathrm{I}_{2}$. 


\subsection{Comparative spectra of the perpendicular and linear isomers and discussion of their binding energies}

The goal of this subsection is to give a comparison of the energy levels and absorption spectroscopy calculated for the two different isomers of $\mathrm{Ar} \cdots \mathrm{I}_{2}$ and to discuss their energetics. The discussion of the spectra will be based on the DIM PT1 surfaces, both for the $X$ (Buchachenko et al., 2000b) and the $B$ [Eq.(4)] states. Note that these surfaces gave very reasonable agreement with the product state distributions from $\mathrm{Ar}+\mathrm{I}_{2}(B)$ vibrationallyinelastic collisions measured in the bulk and in molecular beams (Buchachenko and Stepanov, 1998b). The topology of these PES is illustrated in Fig. 2, while the properties of their minima are presented in Table 1.

The contour plots of the calculated vibrational wave functions for zero total angular momentum (Roncero et al., 2001b) are presented in Fig. 8 for the lowest Van der Waals levels of the $X$ state ( $r$ fixed at its equilibrium value), and of the $(B, v=21)$ states (Buchachenko et al., 2000b; Roncero et al., 2001b). The ground $n_{X}=0$ Van der Waals level of the $X$ state has a dissociation energy of $208.9 \mathrm{~cm}^{-1}$ and corresponds to the Tshaped isomer, as well as the next four levels. The ground state of the linear isomer appears as a degenerate doublet, $n_{X}=5$ and $n_{X}=6$, corresponding to opposite I-I permutation symmetries. Their energy determines the dissociation energy of the linear isomer. The dissociation energies of both isomers are listed in Table 2 together with other theoretical and experimental estimations.

The wave functions of the $(B, v=21)$ levels $n_{B}$ are plotted in the bottom panel of Fig.

8. The lowest level wave functions can be assigned to stretching and bending modes from their nodal pattern, but they become more and more complex and delocalized as energy 
increases. The linear configuration is a saddle point for the $B$-state PES, so only highly excited bending levels have appreciable amplitude density in this region.

The distinct nature of the T-shaped and linear isomers of $\mathrm{Ar} \cdots \mathrm{I}_{2}$ for the DIM PT1 potentials has a direct consequence on $B \leftarrow X$ absorption spectra. Since both the $X$ and $B$ potentials are similar in the region of the T-shaped well, the Franck-Condon principle implies that the transition probability from the $n_{X}=0$ level will be largest for $n_{B}=0$ and will rapidly decrease for higher $n_{B}$. In contrast, the ground level of the linear isomer has significant overlap integrals only with high $n_{B}$ levels whose wave functions are markedly delocalized near the linear configuration.

These trends indeed define the structure of absorption spectra calculated by means of numerically exact line shape and wavepacket methods. Fig. 9 shows the $B \leftarrow\left(X, v^{\prime \prime}=0\right)$ absorption spectra for the $0^{++} \leftarrow 1^{--}$rotational transition obtained with the DIM PT1 $X$ and $B$ PES for the T-shaped and linear isomers (upper and lower panels, respectively) (Roncero et al., 2001b). The spectrum of the T-shaped isomer exhibits a sequence of bands assigned to definite vibrational states $v^{\prime}$ of $\mathrm{I}_{2}(B)$. For each $v^{\prime}$ there are three main lines, corresponding to transitions to the ground and the two first Van der Waals excited levels. This picture is in perfect agreement with all experimental $B \leftarrow X$ absorption spectra (Johnson et al., 1981; Burke and Klemperer, 1993a; Burroughs and Heaven, 2001).

In contrast, the absorption of the linear isomer is mostly continuous. Its intensity rises with energy, but by steps rather than monotonously. These steps correlate with the successive opening of new $\left(B, v^{\prime}\right)$ vibrational manifolds. A broad, quasidiscrete structure is superimposed on this continuous background. It corresponds to the excitation of highly excited intermolecular levels with a wave function sufficiently delocalized to be accessible from the $X$-state linear isomer, see Fig. 8. 
The analysis of the spectral intensity distribution can shed some light on the discussion about the dissociation energy of the isomers presented in section 3.2. The main assumption used by Klemperer and co-workers (Burke and Klemperer, 1993b) to deduce the proportion of the two isomers from the absorption spectra is to assign the discrete part to the T-shaped configuration and the continuous part to the linear configuration. The intensity ratio of the linear and T-shaped isomer absorptions derived from the calculations with the DIM PT1 PES is shown in Fig. 10. It is a highly oscillatory function of energy, with minima and maxima corresponding to the quasidiscrete contributions of the T-shaped and linear isomers, respectively. In between these huge oscillations, one can estimate the base line declining more or less regularly from 20 to 1 . If in addition one takes into account the experimental resolution and the overlapping contributions from many thermally populated rotational levels, the quantitative separation of the two isomers absorption is clearly a very difficult problem.

A rough estimate of the correction to Burke and Klemperer's value can be made as follows. The experimental continuum intensity integrated over the range of the $\mathrm{I}_{2} B \leftarrow X$ $(26,0)$ vibronic band is $2.1 \pm 0.4$ times the integrated intensity of the discrete $B \leftarrow X$ $(26,0)$ band of Ar $\cdots I_{2}$ (Burke and Klemperer, 1993b). This linear to T-shaped intensity ratio can be written as $I_{\exp }(L) / I_{\exp }(T) \simeq\langle I(L) / I(T)\rangle(P(L) / P(T))$ where $\langle I(L) / I(T)\rangle$ is the average intensity ratio (for equal population) of the linear vs T-shaped isomer, and $P(L) / P(T)$ is the linear to T-shaped population ratio at the experimental temperature of about $15 \mathrm{~K}$. The average intensity ratio $\langle I(L) / I(T)\rangle$ can be estimated from Fig. 10 as 10, which would give a population ratio deduced from experiment $P(L) / P(T)=2.1 / 10=0.21$ instead of 3. This may result in a T-shaped isomer which is more bound than the linear one. Although these estimations are rather crude, they give a correction in the right 
direction to reconcile the experimental measurements of $D_{0}(L, X)$ by Stevens Miller et al. and of $D_{0}(T, X)$ by Levy and co-workers. Another source of experimental error could be the saturation of the discrete absorption lines (Klemperer, 2001) in the experiment by Burke and Klemperer (1993b), which could lead to an overestimation of the continuum absorption, hence of the linear:T-shaped population ratio.

\subsection{Final VP product state distributions for both isomers}

The final vibrational product state distribution of $\mathrm{I}_{2}(B)$ fragments obtained for the linear isomer is very broad (Stevens Miller et al., 1999), while that of the T-shaped extends over few vibrational channels, with the first open channel being the most probable one (Johnson et al., 1981). Also, the final rotational distributions obtained from the linear isomer are broad with a maximum at relatively low $j$ values (Burroughs and Heaven, 2001), while that from the T-shaped isomer is very complicated and presents several oscillations as a function of $j$ (Burroughs and Heaven, 2001). This can be understood as an indirect indication of the IVR mediated fragmentation mechanism. These features were qualitatively reproduced by recent quantum calculations (Roncero et al., 2001b) performed with only the $B$ electronic state, thus neglecting the EP dissociation channel.

These differences between the final distributions of $\mathrm{I}_{2}(B)$ fragments are due to important differences in the dynamics. The absorption spectrum from the T-shaped isomer is formed by relatively narrow bands, showing that the dissociation dynamics is rather slow.

For the linear isomer, however, the situation is completely different: the spectrum is very broad hence the dynamics very fast. This is due to the fact that vibrational energy transfer is more efficient at collinear geometries. Also, the initial linear bound states overlap not only with quasi-bound states of $\operatorname{Ar} \cdots \mathrm{I}_{2}\left(B, v^{\prime}\right)$ (essentially those with large probability 
at the collinear geometry) but also with continuum states, which dissociate instantly.

\section{Electronic relaxation and the competition with vibrational relaxation}

\subsection{Competition between Electronic and Vibrational predissociation in the Ar... I $I_{2}$ Van der Waals complex: experimental evidences}

As was already noted, the first experimental evidence of existence of the Ar $\cdots \mathrm{I}_{2}$ Van der Waals complex was obtained by Levy and his team (Kubiak et al., 1978; Levy, 1981). The complex was produced in a supersonic expansion of $I_{2}$ and the fluorescence excitation spectrum was observed, only for vibrational excitation of $\mathrm{I}_{2}(B)$ higher than $v^{\prime}=12$. The Van der Waals laser induced fluorescence (LIF) is an oscillatory function of the $\mathrm{I}_{2}(B)$ vibrational excitation (see Fig. 11). This behavior was interpreted as the result of the competition between vibrational and electronic predissociation. Since only VP produces a fluorescent product, the LIF intensity depends on the relative efficiency of the VP and EP processes, and the vibrational dependence of the LIF intensity is directly related to the vibrational dependence of the branching ratio. It was speculated that in analogy with $\mathrm{He} \cdots \mathrm{I}_{2}$, the VP rate would be a monotonically increasing function of vibrational excitation. The oscillations in the branching ratio would reflect similar ones in the EP rate, induced by changes in the Franck-Condon factors between bound vibrational states of $\mathrm{I}_{2}(B)$ and vibrational continuum states related to some still unidentified repulsive electronic state.

Later experiments confirmed the initial experimental evidence of Kubiak et al. (1978), but contradictory interpretations were proposed. Goldstein et al. (1986) measured absorp- 
tion spectra using intracavity laser spectroscopy (ILS) in conjunction with LIF spectra for the series of $\mathrm{Rg} \cdots \mathrm{I}_{2}$ complexes. The ratio of LIF/ILS intensities provides a direct measurement of the relative population of $\mathrm{I}_{2}$ produced by $\mathrm{VP}$, referenced to the $\mathrm{Rg} \cdots \mathrm{I}_{2}$ population. In other words, it provides a measurement of the VP efficiency with respect to EP, provided that corrections for different Franck-Condon factors for emission and absorption are made. For instance, in the case of $\mathrm{He} \cdots \mathrm{I}_{2}$ where EP is weak, the LIF/ILS ratio was found close to 1: almost all the He $\cdots \mathrm{I}_{2}$ complexes dissociate through VP (Goldstein et al., 1986). In the case of $\mathrm{Kr} \cdots \mathrm{I}_{2}$ or $\mathrm{Xe} \cdots \mathrm{I}_{2}$, only ILS is not negligible. This is an indication that the complex decays predominantly through the EP dark process. Finally, for Ar $\cdots \mathrm{I}_{2}$, the ratio was found to be an oscillatory function of $\mathrm{I}_{2}$ vibrational excitation for $12<v^{\prime}<26$, with a maximum near 0.5 (see Fig. 11). This indicates that EP and VP compete with comparable efficiencies. Although these oscillations are similar to those already observed by Kubiak et al. (1978), Goldstein et al. interpret them as the result of IVR in the VP process, inducing oscillations in the VP rate.

A final experimental confirmation of the results of Kubiak et al. and Goldstein et al. came with a simultaneous measurement of absorption and fluorescence by Burke and Klemperer (1993a) (see Fig. 11). The fluorescence/absorption ratio, corrected by different Franck-Condon factors for emission and absorbtion, yielded a Vibrational Predissociation Efficiency (VPE) which oscillates with the vibrational quantum number similarly to the previous results. In addition, Burke and Klemperer looked at fluorescence intensities corresponding to excitation in the Van der Waals modes, and found VPE which oscillates as a function of $\mathrm{I}_{2}$ stretching similarly to the corresponding VPE for the ground Van der Waals mode. They concluded that the oscillations could not be due to accidental degeneracies in the sparse limit IVR process, but resulted from Franck-Condon factors oscillations in 
the EP process. They also conjectured that the $a 1_{g}$ state is the one responsible for the EP process. Indeed, they noted that among the states which intersect $B\left({ }^{3} \Pi 0_{u}^{+}\right), a 1_{g}$ is the only one to be coupled to $B$ for the strictly T-shaped isomer. Moreover, they noted similarities between the oscillations in the electric field quenching rate of the bare $\mathrm{I}_{2}(B)$ molecule (Dalby et al., 1984) and the ones in the VPE. They concluded that the same $a 1_{g}$ repulsive state must be responsible for both processes.

The experimental results described so far provide information only on relative efficiencies between competing processes, but not on absolute rate constants. These could be obtained in principle from homogeneous linewidths in fluorescence excitation spectra. Unfortunately, these widths are difficult to measure because of rotational congestion. Rough estimates could be obtained for instance for $\mathrm{He} \cdots \mathrm{I}_{2}$ or Ne $\cdots \mathrm{I}_{2}$ (Kenny et al., 1980a), but no published results are available for Ar $\cdots \mathrm{I}_{2}$. However, using picosecond pump-probe techniques, Zewail and his group (Breen et al., 1990; Willberg et al., 1992) measured the time dependence of the nascent $\mathrm{I}_{2}\left(v^{\prime}-3\right)$ population resulting from VP and deduced total Ar $\cdots \mathrm{I}_{2}\left(v^{\prime}\right)$ predissociation rates, including both EP and VP contributions, of $0.014 \mathrm{ps}^{-1}$ for $v^{\prime}=18$ and $0.013 \mathrm{ps}^{-1}$ for $v^{\prime}=21$. Unfortunately, these measurements were restricted to these two $I_{2}$ vibrational excitations.

From these total predissociation rates and branching ratios, Burke and Klemperer (Burke and Klemperer, 1993a) could obtain individual electronic and vibrational predissociation rates for $v^{\prime}=18$ and 21. Assuming that the vibrational predissociation rate increases smoothly and semi-linearly with vibrational excitation, they could extrapolate electronic predissociation rates to the whole range of vibrational excitation from $v^{\prime}=16$ to 24. Thus, they obtained an electronic predissociation rate which oscillates with vibrational excitation, as shown in Fig. 12. 


\subsection{Competition between Electronic and Vibrational predissociation in the Ar... $\mathbf{I}_{2}$ Van der Waals complex : theoretical interpretation}

One of the first goals of the theoretical models was to provide an accurate description for the oscillations of electronic predissociation as a function of vibrational excitation, as obtained from the analysis of Burke and Klemperer (1993a). EP can be well described within the Fermi golden rule approximation as an inital quasibound state $\operatorname{Ar}_{\cdots} \mathrm{I}_{2}(B)$ decaying into a continuum representing the final dissociative electronic state. The zero-order quasibound $\mathrm{Ar} \cdots \mathrm{I}_{2}(B)$ state can be represented by:

$$
\phi_{v^{\prime} n}(r, R, \theta)=\phi_{v^{\prime}}(r) \phi_{n v^{\prime}}(r, \theta)
$$

where $r$ is the I-I distance and $R$ the one from Ar to the $\mathrm{I}_{2}$ center of mass, $\theta$ the angle between the two corresponding Jacobi vectors, $v^{\prime}$ is the initial vibrational exitation of $\mathrm{I}_{2}$ and $n$ a collective quantum number for the Van der Waals modes. The final state of the EP process is some continuum state $\phi_{E f}(r, R, \theta)$ with total energy $E$ associated to a dissociative electronic state $f$ of $I_{2}$. The EP rate is then given by :

$$
k_{E P}^{v^{\prime} n f}=\frac{2 \pi}{\hbar}\left|\left\langle\phi_{v^{\prime}}(r) \phi_{n v^{\prime}}(R, \theta)\left|V_{c}(r, R, \theta)\right| \phi_{E f}(r, R, \theta)\right\rangle\right|^{2}
$$

where $V_{c}(r, R, \theta)$ is the coupling matrix element between the $B$ state and the final repulsive state, and $E$ is the same energy as that of the quasibound state. This equation has been the basis for different approximations. The first one considered the slow Ar as a spectator in the dissociation process: The $R$ and $\theta$ variables are set at their equilibrium values. The predissociation rate is then proportional to the $B-f$ Franck-Condon factors of the $\mathrm{I}_{2}$ molecule. This simple model has been tested for the $B^{\prime \prime} 1_{u}$ and $a 1_{g}$ repulsive states (Roncero et al., 1994a). For the $B^{\prime \prime} 1_{u}$ state, the oscillations of the EP rate as a function of vibrational excitation are much slower than the experimental results (Burke 
and Klemperer, 1993a). This is due to the fact that the short range repulsive portions of the $B$ and $B^{\prime \prime}$ curves are very close and almost parallel, see Fig. 1 . For the $a 1_{g}$ state, the oscillatory pattern is reproduced fairly well (see Fig. 12), provided that the $\mathrm{I}_{2}$ potential curves are shifted by a constant energy correction due to the $\mathrm{Ar}-\mathrm{I}_{2}$ interaction. Modified Franck-Condon simulations taking into account the possibility of vibrational energy transfer due to VP and using the IDIM PT1 PES for all the states involved were later conducted (Buchachenko, 1998). They showed that the $a 1_{g}$ and $12_{g}$ states also produce oscillatory patterns for the EP rate which are close to the experimental ones (Burke and Klemperer, 1993a), casting some doubt on the identification of the $a 1_{g}$ state as the one responsible for the EP process.

One step beyond the spectator model consists in including the motion of the Ar atom in the dynamical treatment. Since the double continuum wave function $\phi_{E f}(r, R, \theta)$ corresponding to the three-body break-up is quite difficult to compute, it is more convenient to obtain the EP rate from a time-dependent wavepacket calculation. In the time-dependent golden rule formalism, the EP rate is given by (Villarreal et al., 1991):

$$
k_{E P}^{v^{\prime} n f}=\frac{1}{\hbar^{2}} \int_{-\infty}^{+\infty} d t e^{i E t / \hbar}\left\langle\Phi_{v^{\prime} n f}(r, R, \theta, t=0) \mid \Phi_{v^{\prime} n f}(r, R, \theta, t)\right\rangle
$$

where the time dependent wave-packet $\Phi_{v^{\prime} n}(r, R, \theta, t)$ is propagated on the final dissociative surface $f$ from the initial condition : $\Phi_{v^{\prime} n f}(r, R, \theta, t=0)=V_{c}(r, R, \theta) \phi_{v^{\prime}}(r) \phi_{n v^{\prime}}(R, \theta)$. Two-dimensional fixed $\theta$ (Roncero et al., 1994b) and full three-dimensional time dependent golden rules calculations (Roncero et al., 1996) both gave oscillations of the EP rate with $v^{\prime}$ which had the same period as the experimental ones if $a 1_{g}$ was the final dissociative state. A good agreement with the absolute position of the oscillations was obtained with a Van der Waals well of $100 \mathrm{~cm}^{-1}$ on the $a 1_{g}$ potential energy surface, and the absolute values of the EP rates were in good agreement with the experimental ones for an interstate 
$B-a$ coupling of the order of $14 \mathrm{~cm}^{-1}$.

The Golden rule treatments of electronic predissociation are perturbative models assuming that the VP process which occurs on the $B$ electronic potential energy surface is not affected by the competing electronically non-adiabatic process. The competition between EP and VP, which are of similar efficiency for $12<v^{\prime}<26$, may induce non perturbative effects. For instance, one may suppose that the broadening of the resonant bright and dark states due to electronic coupling may influence the IVR process. Thus, several attemps were made to simultaneously study vibrational and electronic predissociation. The first approach (Buchachenko, 1998) used classical dynamics to describe VP and surface hopping (with Landau-Zener probabilities) localised at crossing seams between electronic potentials to describe EP. This study was done on IDIM PT1 potential energy surfaces (see section 3.4) and showed that all the final electronic states coupled to the $B$ one contribute to EP. However, this model did not reproduce the oscillations in the VP efficiencies observed by Burke and Klemperer (1993a). Bastida et al. (1999) used a quantum description of the $\mathrm{I}_{2}$ vibration and a classical description of the Van der Waals modes for the VP process. The EP process was described by surface hopping, the probability being given by Franck-Condon factors. The potentials used were taken from (Roncero et al., 1996). This model produced a good agreement with the experimental VPE. In addition, it was shown that first order rate equations are not adequate to describe the time dependence of product populations. This was due to the dependence of the electronic predissociation rate on the vibrational quantum numbers of the intermediate states of the process.

More recently, a full global quantum model including simultaneaously the VP and EP processes was performed using the DIM PT1 model for the $B$ and the four coupled 
electronic states involved, $a 1_{g}, a^{\prime} 0_{g}^{+}, B^{\prime \prime} 1_{u}$, and $12_{g}$ (Lepetit et al., 2002). In agreement with (Buchachenko, 1998), it was shown that all the dissociative electronic channels except $B^{\prime \prime} 1_{u}$ could contribute significantly to EP. The total predissociation rates obtained for $v^{\prime}=18$ and 21 were in very good agreement with the real time measurements of Zewail and co-workers (Breen et al., 1990; Willberg et al., 1992) (see Fig. 13). The calculated VPE oscillations were similar to the experimental ones, without any adjustment of the potentials. However, according to these calculations, the main source of oscillations comes from the VP rate, which is strongly influenced by IVR in the sparse limit. The EP rate is a rather smooth function of vibrational excitation. Indeed, each $\mathrm{I}_{2}$ electronic repulsive state produces a partial EP rate which oscillates strongly according to its Franck-Condon factors to the $B$ state. However, the oscillations of each of the three contributing electronic states are out of phase, so that the total EP rate, is much smoother than each of its three contributions.

Lepetit et al. (2002) also studied the electronic predissociation of the linear isomer at the same level of theory. The distinct nature of the VP process makes the EP mechanism for the linear isomer different from that of the T-shaped one. Vibrational predissociation of the linear isomer is impulsive and fast, so that the argon atom rapidly leaves the interaction region without making large excursions out of the region of the collinear arrangement. Thus, EP has a lower probability and, in contrast to the T-shaped case, it proceeds almost exclusively through the $a^{\prime} 0_{g}^{+}$state which is the only one coupled to the $B$ state in the linear geometry. It would be nice to obtain experimental confirmation for this mechanism.

To summarize the situation on the electronic and vibrational predissociation of $\mathrm{Ar} \cdots \mathrm{I}_{2}$, it must be admitted that after more than two decades of intense studies there is not yet 
a clear consensus on:

- which are the $\mathrm{I}_{2}$ electronic states responsible for EP: the single $a 1_{g}$ state, or a collaborative effect of several repulsive states?

- what is the origin of the VPE oscillations as of function of vibrational excitation: Franck-Condon factors inducing oscillations in the EP rates, or sparse limit IVR inducing oscillations in the VP rates?

One way to definitely solve this puzzle would be to undertake measurements of the individual electronic and vibrational predissociation rates, and not only of the VPE, which is now well established. This could be achieved by high resolution spectroscopy or by real time measurements, limited so far to two vibrational excitations.

\section{Conclusions and Perspectives}

At the end of this review, it appears clearly that although much progress has been made in the comprehension of the dynamical processes for the Ar $\cdots \mathrm{I}_{2}$ Van der Waals complex, much is still awaiting to be learned. There is nowadays little doubt that two isomers of the complex can coexist, and that the linear isomer is responsible for the cage effect on $\mathrm{I}_{2}$. There is little uncertainty on the dissociation energy of the T-shaped isomer. However, no clear consensus has been found yet on the energy of the linear isomer, and $a b$ initio calculations have not yet reached a sufficient accuracy on the determination of these dissociation energies to decide whether the linear or the T-shaped isomer is the more bound one. Excitation of the perpendicular isomer to the $B$ state produces long-lived resonance states and sharp lines in photodissociation spectra. Excitation of the linear isomer leads to 
a fast dissociation dynamics and a broad background on the spectra. It is well established that the dissociation of the T-shaped isomer implies the loss of several vibrational quanta for $\mathrm{I}_{2}$, and that it occurs as a stepwise Intramolecular Vibrational Relaxation process. However, there is no final agreement on the regime of this process: sparse, as obtained by all quantum calculations, or statistical? Electronic predissociation is clearly the result of couplings between the $B$ state and dissociative states induced by the presence of the Ar atom. But which dissociative state(s)? There is a consensus on the fact that there is a competition between electronic and vibrational predissociation, which induces oscillations as a function of vibrational excitation in the relative efficiencies of these processes. But what is the process responsible for these oscillations? One tentative interpretation is that the $B$ state is coupled to the $a 1_{g}$ dissociative one and that oscillations appear in the EP rate as a result of varying Franck-Condon factors. Another competing explanation is that the $B$ state is coupled to a set of several dissociative states, and that oscillations appear in the VP rate as a result of IVR in the sparse regime.

So what should be done to achieve complete understanding of this prototype system? On the theoretical side, priority should be put on the production of high quality potentials. From the ab initio point of view, the most important steps to be taken next would be the explicit treatment of the spin-orbit interaction and the calculations of excited electronic states (first of all, the $B$ one) and couplings. These steps are quite demanding and will require to switch to another strategy - from the single-reference coupled cluster method, which is the method of choice for the ground state due to its inherent ability to accurately determine intermolecular interactions, to multiconfigurational methods which must carefully take into account intramolecular interactions describing the internal structure of the monomer. Both effects are equally important for the excited Ar $\cdots \mathrm{I}_{2}$ system, and 
it may well be that $a b$ initio methods capable of achieving a precise description are yet to be developed. The refinement of the DIM approach will require the use of extended basis of atomic states covering the interaction with the ion-pair states and valence-excited manifolds of the molecular halogen. These new highly accurate potentials should be the starting points of dynamical calculations, the accuracy of which is nowadays only limited by the one of the electronic potentials. On the experimental side, it is striking to note that, although intense studies have been performed over decades, little is known on absolute values of total $(\mathrm{EP}+\mathrm{VP})$ decay rates as a function of initial excitation, whereas relative efficiencies of EP versus VP is well established. These total decay rates would bring much light on two open questions: one on the IVR regime (sparse/statistical) for VP, the other on the EP/VP competition (are oscillations on relative efficiencies induced by similar ones on EP or on VP?).

Another interesting perspective is to move to higher excitation energies above the valence manifold of the Ar $\cdots \mathrm{I}_{2}$ complex. Theoretical studies of the Rydberg states converging to the positive ion threshold and studies of the cation itself in connection with experiments by Donovan group (Cockett et al., 1993, 1994; Goode et al., 1994; Cockett et al., 1996) may provide additional precise information on the energetics of the complex in the ground state. Another challenging subject is the study of the Ar $\cdots \mathrm{I}_{2}$ complex excited to ion-pair states accessible via single- or multi-photon transitions (Brand and Hoy, 1987; Lawley and Donovan, 1993). As follows from numerous collisional studies (Lawley, 1988; Urbachs et al., 1993; Akopyan et al., 1999; Teule et al., 1999; Akopyan et al., 2001; Fecko et al., 2001; Bibinov et al., 2002; Fecko et al., 2002), interaction with a rare gas atom induces efficient non-adiabatic vibronic transitions between closely lying states of distinct symmetry. This implies that in the complex, electronic predissociation (to bound $\mathrm{I}_{2}$ electronic 
states in this case) will compete with vibrational predissociation. The proofs for such expectations can be found in the experimental study of large argon clusters (Fei et al., 1992). Recent calculations of the diabatic PES's and couplings in the frame of DIM models similar to those described here for valence states (Batista and Cocker, 1997; Tscherbul et al., in press) provide the grounds for future theoretical studies of the dynamics.

\section{Acknowledgements}

We owe much from numerous discussions with active researchers in this field over the past decades. It is not possible to mention all of them, but we wish to acknowledge fruitful interactions with J.A. Beswick, M.C. Heaven, K.C. Janda, W. Klemperer and C. Meier. We also wish to acknowledge financial supports which, over the past few years, allowed us to interact closely and made the present work possible : INTAS 97-31573 and PICASSO HF1999-0132 international grants, national funding from AAB Russian Foundation for Basic Research (grant 02-03-32676), Ministerio de Ciencia y Tecnología (grant No. BFM2001-2179) and CNRS. Allocation of CPU time from the "Institut du Développement et des Ressources Informatiques" is also gratefully acknowledged. 
Appendix I: Details of the DIM method to determine the Ar $\cdots I_{2}$ PES and couplings

The grounds of the DIM method are well described in the literature (e.g. Tully, 1977; Kuntz, 1979, 1982). It can be implemented in a variety of ways. Here the particular formulation relevant to the Ar $\cdots \mathrm{I}_{2}$ complex is presented.

The total electronic Born-Oppenheimer Hamiltonian is expressed as a sum of the Hamiltonians corresponding to diatomic and atomic fragments of the system:

$$
\hat{H}=\hat{H}_{I_{2}}+\hat{H}_{A r I_{a}}+\hat{H}_{A r I_{b}}-\hat{H}_{I_{a}}-\hat{H}_{I_{b}}-\hat{H}_{A r}
$$

where $a$ and $b$ label iodine atoms. It can be recast into two terms, $\hat{H}_{0}$ describing the isolated fragments and $\hat{H}_{1}$ their interaction:

$$
\begin{gathered}
\hat{H}_{0}=\hat{H}_{I_{2}}+\hat{H}_{A r}, \\
\hat{H}_{1}=\hat{H}_{1 a}+\hat{H}_{1 b}=\left(\hat{H}_{A r I_{a}}-\hat{H}_{I_{a}}-\hat{H}_{A r}\right)+\left(\hat{H}_{A r I_{b}}-\hat{H}_{I_{b}}-\hat{H}_{A r}\right) .
\end{gathered}
$$

The conventional DIM approach implies the variational solution of the Schrödinger equation for the total Hamiltonian, whereas the DIM perturbation theory approximation suggested independently by Naumkin (1991) and by Buchachenko and Stepanov (1996b) treats $\hat{H}_{1}$ in Eq.(12) as a perturbation.

The main feature of the DIM approach is the use of polyatomic basis functions (PBF) constructed as linear combinations of many-electron functions centered on each atom and 
describing atomic states which contribute to the electronic configuration of the whole system. A minimum set of 36 PBF's can be defined as :

$$
\phi_{k}=\phi_{a_{i} b_{j}}=\chi^{A r} \chi_{i}^{a} \chi_{j}^{b}
$$

It includes six spin-orbital functions describing the ${ }^{2} \mathrm{P}$ multiplet centered on each $I$ atom $\chi_{i}^{\alpha}, \alpha=a, b$ and one function $\chi^{A r}$ describing the ${ }^{1} \mathrm{~S}$ state of Ar. This PBF set is assumed to form an orthonormal basis. Two attempts to take into account the contribution from higher ion-pair states of the iodine gave controversial results (Grigorenko et al., 1997a; Naumkin and McCourt, 1998b).

Using the basis set $\phi_{k}$ of Eq.(13), it is easy to evaluate the matrix elements of the diatomic fragment Hamiltonians contributing to $\hat{H}_{1}$ in Eq.(12). In the reference frame related to the $\mathrm{I}_{\alpha}-\mathrm{Ar}$ axis $\mathcal{R}_{\alpha}$, the $\hat{H}_{A r I_{\alpha}}$ matrix has a particularly simple form and is

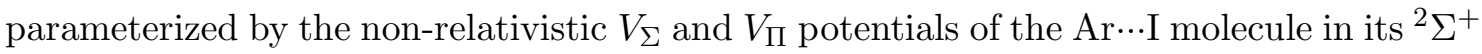
and ${ }^{2} \Pi$ state, respectively. It is convenient to choose the $I_{2}$ axis $\mathbf{r}$ as the common reference frame. The transformation from the $\mathcal{R}_{\alpha}$ to the $\mathbf{r}$ frame is given by the standard rotation matrices $D_{\alpha}\left(0, \beta_{\alpha}, 0\right)$ (Wigner rotation matrices or direction cosine matrices, depending on particular choice of $\mathrm{ABF}$ ) in which only one angle $\beta_{\alpha}$ is non-zero.

Constructing the $\hat{H}_{0}$ matrix in the PBF set is more involved due to the complexity of the $\mathrm{I}_{2}$ electronic structure. Its eigenfunctions

$$
\hat{H}_{0} \psi_{n}=u_{n} \psi_{n}
$$

where $n$ enumerates the adiabatic electronic states of $\mathrm{I}_{2}$ and $u_{n}$ are the corresponding energies as a function of $r$, are expressed in terms of the PBF

$$
\psi_{n}(r)=\sum_{k} C_{k}^{n}(r) \phi_{k}
$$


The total Hamiltonian matrix is therefore

$$
\mathbf{H}=\mathbf{u}+\mathbf{C}^{\dagger}\left(\mathbf{D}_{a}^{\dagger} \mathbf{H}_{A r I_{a}} \mathbf{D}_{a}+\mathbf{D}_{b}^{\dagger} \mathbf{H}_{A r I_{b}} \mathbf{D}_{b}-\mathbf{H}_{I_{a}}-\mathbf{H}_{I_{b}}\right) \mathbf{C}
$$

where $\mathbf{u}$ is a diagonal matrix containing the energy curves of $\mathrm{I}_{2}$, and $\mathbf{H}_{I_{\alpha}}$ are diagonal matrices containing the energies of the atomic iodine terms (vanishing in the non-relativistic case).

The different DIM approaches correspond to different representations of the atomic basis functions, different approximations for the eigenfunctions of $\hat{H}_{0}$ [Eqs.(11) and (15)], and to two different ways of treating $\hat{H}_{1}$ [Eq.(11) and the 2 nd term of the sum in Eq.(16)]: exactly or as a perturbation. These approaches are discussed in section 3.4. 


\section{Appendix II : classification of IVR regimes}

The different regimes of IVR, from sparse to statistical, can be modelled in terms of an infinite collection of non-interacting bound states (see Fig. 14), with energies $E_{n}=n \Delta$, $\mathrm{n}=-\infty, \ldots, \infty$, coupled to a dissociative continuum, so that their halfwidth is equal to $\Gamma$ for each of them (Roncero et al., 1997), in close correspondence to the treatment of Bixon and Jortner (1969). It can be considered that this ensemble of first-order bound states arises from the diagonalization of an initial zero-order "bright" state coupled (by a constant quantity $V$ ) to an infinite ensemble of zero-order "dark" states. Therefore, the initial wavepacket can be expanded in terms of these states with a weight ratio $a_{n} / a_{0}=$ $V /(n \Delta-i \Gamma)$ (Roncero et al., 1997). This model leads to a natural classification, as previously discussed (Bixon and Jortner, 1969; Freed and Nitzan, 1980; Uzer, 1991; Miller, 1991), of IVR dynamics in terms of the $\Gamma / \Delta$ ratio as:

(a) Sparse regime, $(\Gamma<<\Delta)$ : The resonances are well separated (see Figs. 15 and 16). The initially populated bright state will usually interact with one or a few dark states, so that quantum phenomena such as recurrences are readily apparent. In some cases, the zero-order bound states are indirectly coupled through their mutual coupling to the dissociative continua (Roncero et al., 1993; Roncero and Gray, 1996; Roncero et al., 1997).

(b) Intermediate regime, $(\Gamma \approx \Delta)$ : In this regime the resonances are mixed but have not completely lost their individual identity (see Figs. 15 and 16). This will be apparent both in the spectrum, in which nearby transitions will overlap with each other, and in the dynamics, which will exibit non-exponential decay with weak recurrences in the population of the initially excited state. 
(c) Statistical regime, $(\Gamma>>\Delta)$ : In this regime there are so many closely spaced resonances that they blend together to yield a quasi-Lorentzian excitation spectrum. As a consequence, the initial states lose their identity and decay irreversibly as a single exponential.

In the sparse and intermediate regimes the VP rate is expected to oscillate as a function of $v^{\prime}$ (except if there is a smoothing out due to averaging as will be commented below), and the only regime in which the desire smooth monotonic behaviour is expected is the statistical one. This regime is traditionally attributed to large molecules and it is interesting to know if it can occur in such small molecular systems.

The statistical limit can be obtained by increasing the density of states (i.e. decreasing the spacing $\Delta$ ) and/or the width of each individual state, $\Gamma$, but also by varying the total energy spreading of the spectrum, which depends enormously on the initial state. 


\section{References}

Akopyan, M. E., Bibinov, N. R., Kokh, D. B., Pravilov, A. M., Stepanov, M. B., and Vasyutinskit, O. S., 1999, Chem. Phys., 242, 263.

Akopyan, M. E., Bibinov, N. K., Kokh, D. B., Pravilov, A. M., Sharova, O. L., and Stepanov, M. B., 2001, Chem. Phys., 263, 459.

Alfano, J. C., Kliner, D., Johnson, A. E., Levinger, N. E., and Barbara, P. F., 1992, in Ultrafast Phenomena VIII, edited by J. L. Martin, A. Migus, G. A. Mourou, and A. H. Zewail (Springer, New York), p. 653.

Apkarian, V. A., and Schwentner, N., 1999, Chem. Rev., 99, 1481.

Aquilanti, V., Luzzatti, E., Pirani, F., and Volpi, G. G., 1988, J. Chem. Phys., 89, 6165.

Aquilanti, V., Candori, R., Cappelletti, D., Luzzatti, E., and Pirani, F., 1990, Chem. Phys., 145, 293.

Aquilanti, V., Cappelletti, D., Lorent, V., Luzzatti, E., and Pirani, F., 1993, J. Phys. Chem., 97, 2063.

Arnot, C., and McDowell, C., 1958, Can. J. Chem., 36, 114.

Asmis, K. R., Taylor, T. R., Xu, C., and Neumark, D. M., 1998, J. Chem. Phys., 109, 4389.

Baba, H., and Sakurai, K., 1985, J. Chem. Phys., 82, 4977.

Bastida, A., Zúñiga, J., Requena, A., Sola, I., Halberstadt, N., and Beswick, J. A., 1997, Chem. Phys. Lett., 280, 185. 
Bastida, A., Zúñiga, J., Requena, A., Halberstadt, N., and Beswick, J. A., 1999, Chem. Phys., 240, 229.

Bastida, A., Zúñiga, J., Requena, A., Miguel, B., Beswick, J. A., Vigué, J., and Halberstadt, N., 2002, J. Chem. Phys., 116, 1944.

Batista, V. S., and Cocker, D. F., 1997, J. Chem. Phys., 106, 6923.

Batista, V. S., and Coker, D. F., 1996, J. Chem. Phys., 105, 4033.

Baumfalk, R., Nahler, N. H., and Buck, U., 2001, Faraday Discuss., 118, 247.

Becker, C. H., Casavecchia, P., and Lee, Y. T., 1979, J. Chem. Phys., 70, 2986.

Beeken, P. B., Hanson, E. A., and Flynn, G. W., 1983, J. Chem. Phys., 78, 5892.

Ben-Nun, M., Levine, R. D., Jonas, D. M., and Fleming, G. R., 1995, Chem. Phys. Letters, 245, 629 .

Benderskit, A. V., Zadoyan, R., and Apkarian, V. A., 1997, J. Chem. Phys., 107, 8437.

Beswick, J. A., and Jortner, J., 1977, Chem. Phys. Lett., 49, 13.

Beswick, J. A., and Jortner, J., 1978a, J. Chem. Phys., 69, 512.

Beswick, J. A., and Jortner, J., 1978b, J. Chem. Phys., 68, 2277.

Beswick, J. A., and Jortner, J., 1980, Mol. Phys., 39, 1137.

Beswick, J. A., and Jortner, J., 1981, Adv. Chem. Phys., 47, 363.

Beswick, J. A., Monot, R., Philippoz, J., and van den Bergh, H., 1987, J. Chem. Phys., 86, 3965. 
Bibinov, N. R., Malinina, O. L., Pravilov, A. M., Stepanov, M. B., and Zakharova, A. A., 2002, Chem. Phys., 277, 179.

Bixon, M., and Jortner, J., 1969, J. Chem. Phys., 50, 3284.

Blazy, J. A., DeKoven, B. M., Russell, T. D., and Levy, D. H., 1980, J. Chem. Phys., $72,2439$.

Borrmann, A., Li, Z., and Martens, C. C., 1993, J. Chem. Phys., 98, 8514.

Brand, J. C. D., and Hoy, A. R., 1987, Appl. Spectrosc. Rev., 23, 285.

Breen, J. J., Willberg, D. M., Gutmann, M., and Zewail, A. H., 1990, J. Chem. Phys., 93, 9180.

Brown, F. B., Schwenke, D. W., and Truhlar, D. G., 1985, Theor. Chim. Acta, 68, 23.

Brown, R. L., and Klemperer, W., 1964, J. Chem. Phys., 41, 3072.

Broyer, M., Vigué, J., and Lehmann, J. C., 1975, J. Chem. Phys., 63, 5428.

Broyer, M., Vigué, J., and Lehmann, J. C., 1976, J. Chem. Phys., 64, 4793.

Buchachenko, A. A., 1998, Chem. Phys. Lett., 292, 273.

Buchachenko, A. A., and Stepanov, N. F., 1993, J. Chem. Phys., 98, 5486.

Buchachenko, A. A., and Stepanov, N. F., 1996a, Chem. Phys. Lett., 261, 591.

Buchachenko, A. A., and Stepanov, N. F., 1996b, J. Chem. Phys., 104, 9913.

Buchachenko, A. A., and Stepanov, N. F., 1997a, J. Chem. Phys., 106, 4358.

Buchachenko, A. A., and Stepanov, N. F., 1997b, J. Chem. Phys., 106, 10134. 
Buchachenko, A. A., and Stepanov, N. F., 1998a, Russ. J. Phys. Chem., 72, 69.

Buchachenko, A. A., and Stepanov, N. F., 1998b, Russ. J. Phys. Chem., 72, 390.

Buchachenko, A. A., González-Lezana, T., Hernández, M. I., Delgado-Barrio, G., and Villarreal, P., 2000a, Chem. Phys. Lett., 318, 578.

Buchachenko, A. A., Roncero, O., and Stepanov, N. F., 2000b, Russian J. Chem. Phys., 74, Suppl.2, S193.

Buchachenko, A. A., Krems, R. V., Szczȩśniak, M. M., Xiao, Y.-D., Viehland, L. A., and Chąasiński, G., 2001, J. Chem. Phys., 114, 9919.

Buchachenko, A. A., Prosmiti, R., Cunha, C., Delgado-Barrio, G., and VillarREAL, P., 2002, J. Chem. Phys., 117, 6117.

Burcl, R., Krems, R. V., Buchachenko, A. A., SzczȩŚniak, M. M., Chałasiński, G., and Cybulski, S. M., 1998, J. Chem. Phys., 109, 2144.

Burde, D. H., McFarlane, R. A., and Wiesenfeld, J. R., 1974, Phys. Rev. A, 10, 1917.

Burke, M. L., and Klemperer, W., 1993a, J. Chem. Phys., 98, 6642.

Burke, M. L., and Klemperer, W., 1993b, J. Chem. Phys., 98, 1797.

Burroughs, A., and Heaven, M. C., 2001, J. Chem. Phys., 114, 7027.

Burroughs, A., van Marter, T., and Heaven, M. C., 1999, J. Chem. Phys., 111, 2478.

Burroughs, A., Kerenskaya, G., and Heaven, M. C., 2001, J. Chem. Phys., 115, 784.

Capelle, G. A., and Broida, H. P., 1973, J. Chem. Phys., 58, 4212. 
Casavecchia, P., He, G., Sparks, R., and Lee, Y. T., 1982, J. Chem. Phys., 77, 1878.

Castleman, Jr., A. W., 1992, in Clusters of Atoms and Molecules, edited by H. Haberland (Springer Verlag, New York), cited in papan:93, papan:92, "Molecular clusters offer a unique environment in which to study the effects of increasing solvation on chemical systems/reaction dynamics", textbf à rechercher.

Chazasiński, G., and Szczȩśniak, M. M., 1994, Chem. Rev., 94, 1723.

Chatasiński, G., Gutowski, M., Szczȩśniak, M. M., Sadlej, J., and Scheiner, S., 1994, J. Chem. Phys., 101, 6800.

Chergui, M., and Schwentner, N., 1992, Trends Chem. Phys., 2, 89.

Cline, J. I., Evard, D. D., Reid, B. P., Sivakumar, N., Thommen, F., and Janda, K. C., 1987, in Sructure and Dynamics of Weakly-Bound Molecular Systems, edited by A. Weber (Reidel, Dordrecht), p. 533.

Cockett, M., Goode, J. G., Lawley, K. P., and Donovan, R. J., 1993, Chem. Phys. Lett., $214,27$.

Cockett, M., Goode, J. G., Maier, R. R. J., Lawley, K. P., and Donovan, R. J., 1994, J. Chem. Phys., 101, 126.

Cockett, M., Beattie, D. A., Donovan, R. J., and Lawley, K. P., 1996, Chem. Phys. Lett., 259, 554 .

Conley, A. J., Fang, J.-Y., and Martens, C. C., 1997, Chem. Phys. Lett., 272, 103.

Cybulski, S. M., and Holt, J. S., 1999, J. Chem. Phys., 110, 7745. 
Cybulski, S. M., Burcl, R., Chałasiński, G., and Szczȩśniak, M. M., 1995, J. Chem. Phys., 103, 10116.

Dalby, F. W., Levy, C., and Vanderlinde, J., 1984, Chem. Phys., 85, 23.

De Jong, W. A., Visscher, L., and Nieuwpoort, W. C., 1997, J. Chem. Phys., 107, 9046.

Delaney, N., Faeder, J., and Parson, R., 1999, J. Chem. Phys., 111, 651.

Delgado-Barrio, G., Villarreal, P., Mareca, P., and Albelda, G., 1983, J. Chem. Phys., 78, 280.

Derouard, J., and Sadeghi, N., 1984a, Chem. Phys., 88, 171.

Derouard, J., and Sadeghi, N., 1984b, J. Chem. Phys., 81, 3002.

Dexheimer, S. L., Durand, M., Brunner, T. A., and Pritchard, D. E., 1982, J. Chem. Phys., 76, 4996.

Dexheimer, S. L., Brunner, T. A., and Pritchard, D. E., 1983, J. Chem. Phys., 79, 5206.

Drabe, K. E., and van Voorst, J. D. W., 1985, Chem. Phys., 99, 135.

Drabe, K. E., De Groot, A., and van Voorst, J. D. W., 1985a, Chem. Phys., 99, 121.

Drabe, K. E., Langelaar, J., Bebelaar, D., and van Voorst, J. D. W., 1985b, Chem. Phys., 97, 411.

Du, H., Krajnovich, D. J., and Parmenter, C. S., 1991, J. Chem. Phys., 95, 2104.

Evard, D. D., Thommen, F., and Janda, K. C., 1986, J. Chem. Phys., 84, 3630. 
Evard, D. D., Bieler, C. R., Cline, J. I., Sivakumar, N., and Janda, K. C., 1988a, J. Chem. Phys., 89, 2829.

Evard, D. D., Cline, J. I., and Janda, K. C., 1988b, J. Chem. Phys., 88, 5433.

Ewing, G. E., 1979, J. Chem. Phys., 71, 3143.

EwIng, G. E., 1980, J. Chem. Phys., 72, 2096.

EwING, G. E., 1982, Faraday Disc. Chem. Soc., 73, 402.

EwIng, G. E., 1986, J. Phys. Chem., 90, 1990.

Fang, J., and Martens, C. C., 1996, J. Chem. Phys., 105, 9072.

Fecko, C. J., Freedman, M. A., and Stephenson, T. A., 2001, J. Chem. Phys., 115, 4132.

Fecko, C. J., Freedman, M. A., and Stephenson, T. A., 2002, J. Chem. Phys., 116, 1361.

Fei, S., Zheng, X., Heaven, M. C., and Tellinghuisen, J., 1992, J. Chem. Phys., 97, 6057.

Franck, J., and Rabinovitch, E., 1934, Trans. Far. Soc., 30, 120.

Franck, J., and Wood, R. W., 1911, Philos. Mag. (ser. 6), 21, 314.

Freed, K., and Nitzan, A., 1980, J. Chem. Phys., 73, 4765.

Gardner, D. J., and Preston, S. R., 1992, J. Chem. Eng. Data, 37, 500.

Gentry, W. R., 1984, J. Chem. Phys., 81, 5737. 
Gerber, R. B., McCoy, A. B., and Garcia-Vela, A., 1994, Annu. Rev. Phys. Chem., $45,275$.

Gersonde, I. H., and Gabriel, H., 1993, J. Chem. Phys., 98, 2094.

Gerstenkorn, S., and Luc, P., 1985, J. Phys. (Paris), 46, 865.

Goldfield, E. M., and Gray, S. K., 1997a, J. Chem. Soc. Faraday Trans., 93, 909.

Goldfield, E. M., and Gray, S. K., 1997b, Chem.Phys.Lett., 276, 1.

Goldstein, N., Brack, T. L., and Atkinson, G. H., 1986, J. Chem. Phys., 85, 2684.

González-Lezana, T., Hernández, M. I., Delgado-Barrio, G., Buchachenko, A. A., and Villarreal, P., 1996, J. Chem. Phys., 105, 7454.

Goode, J. G., Cockett, M. C. R., Lawley, K. P., and Donovan, R. J., 1994, Chem. Phys. Lett., 231, 521.

Gray, S. K., 1992, Chem. Phys. Lett., 197, 86.

Gray, S. K., and Rice, S. A., 1986, Faraday Disc. Chem. Soc., 82, 307.

Gray, S. K., and Roncero, O., 1995, J. Phys. Chem., 99, 2512.

Gray, S. K., Rice, S. A., and Davis, M. J., 1986, J. Phys. Chem., 90, 3470.

Greenblatt, B. J., Zanni, M. T., and Neumark, D. M., 1997, Faraday Discuss., 108, 101.

Grigorenko, B. L., Nemukhin, A. V., and Apkarian, V. A., 1997a, Chem. Phys., 219, 161. 
Grigorenko, B. L., Nemukhin, A. V., Buchachenko, A. A., Stepanov, N. F., and Umanskit, S. Y., 1997b, J. Chem. Phys., 106, 4575.

Halberstadt, N., and Beswick, J. A., 1982, Faraday Disc. Chem. Soc., 73, 357.

Halberstadt, N., Beswick, J. A., Roncero, O., and Janda, K. C., 1992a, J. Chem.Phys., 96, 2404.

Halberstadt, N., Serna, S., Roncero, O., and Janda, K. C., 1992b, J. Chem.Phys., 97,341 .

Hall, G., McAuliffe, M. J., Giese, C. F., and Gentry, W. R., 1983, J. Chem. Phys., 78,5260 .

Harris, A. L., Brown, J. K., and Harris, C. B., 1988, Annu. Rev. Phys. Chem., 39, 341.

Harris, S. J., Novick, S. E., Klemperer, W., and Falconer, W. E., 1974, J. Chem. Phys., 61, 193.

Hernández, M. I., González-Lezana, T., Delgado-Barrio, G., Villarreal, P., and Buchachenko, A. A., 2000, J. Chem. Phys., 113, 4620.

HiLl, T., 1946, J. Chem. Phys., 14, 465.

Hirschfelder, J. O., Curtiss, C. M., and Bird, R. B., editors, 1954, Molecular Theory of Gases and Liquids, 2nd edition (Wiley, New York).

Hu, X., and Martens, C. C., 1993a, J. Chem. Phys., 99, 9532.

Hu, X., and Martens, C. C., 1993b, J. Phys. Chem., 98, 8551. 
Huang, S. S., Bieler, C. R., Tao, F.-M., Klemperer, W., Casavecchia, P., Volpi, G. G., Janda, K. C., and Halberstadt, N., 1995, J. Chem. Phys., 102, 8846.

Huber, K. P., and Herzberg, G., editors, 1979, Molecular Spectra and Molecular Structure. IV. Constants of Diatomic Molecules (Van Nostrand, New York).

J.A. Beswick, G. Delgado-Barrio and J. Jortner, 1979, J. Chem. Phys., 70, 3895.

Jahn, D. G., Clement, S. G., and Janda, K. C., 1994, J. Chem. Phys., 101, 283.

Jahn, D. G., Barney, W. S., Cabalo, J., Clement, S., Slotterback, T. J., Janda, K. C., and Halberstadt, N., 1996, J. Chem. Phys., 104, 3501.

Johnson, K. E., Wharton, L., and Levy, D. H., 1978, J. Chem. Phys., 69, 2719.

Johnson, K. E., Sharfin, W., and Levy, D. H., 1981, J. Chem. Phys., 74, 163.

Jungwirth, P., Fredu, E., and Gerber, R. B., 1996, J. Chem. Phys., 104, 1.

Kajimoto, O., and Fueno, T., 1972, Bull. Chem. Soc. Japan, 45, 99.

Katô, H., and BaвA, M., 1995, Chem. Rev., 95, 2311.

Kenny, J. E., Johnson, K. E., Sharfin, W., and Levy, D. H., 1980a, J. Chem. Phys., $72,1109$.

Kenny, J. E., Russell, T. D., and Levy, D. H., 1980b, J. Chem. Phys., 73, 3607.

Kitaigorodski,, A. I., 1951, Izv. AN SSSR Ser. Fiz. (Bulletin of Academy of Sciences USSR, Phys. Ser., 15, 157.

Klemperer, W., 2001, (private communication).

Kokubo, T., and Fujimura, Y., 1986, J. Chem. Phys., 85, 7106. 
Krajnovich, D. J., Butz, K. W., Du, H., and Parmenter, C. S., 1989a, J. Chem. Phys., 91, 7705.

Krajnovich, D. J., Butz, K. W., Du, H., and Parmenter, C. S., 1989b, J. Chem. Phys., 91, 7725.

Kubiak, G., Fitch, P., Wharton, L., and Levy, D. H., 1978, J. Chem. Phys., 68, 4477. Kuntz, P. J., 1979, in Atom-Molecule Collision Theory, edited by R. B. Bernstein (Plenum Press, New York), p. 79.

Kuntz, P. J., 1982, Ber. Bunsenges. Phys. Chem., 86, 367.

Kunz, C. F., Burghardt, I., and Hess, B., 1998, J. Chem. Phys., 109, 359.

Kurzel, R. B., and Steinfeld, J. I., 1970, J. Chem. Phys., 53, 3293.

Kurzel, R. B., Steinfeld, J. I., Hatzenbuhler, D. A., and Leroi, G. E., 1971, J. Chem. Phys., 55, 4822.

L. J. van der Burgt, J. P. N., and Heaven, M. C., 1984, J. Chem. Phys., 81, 5514.

Lara-Castells, M. P. D., Krems, R. V., Buchachenko, A. A., Delgado-Barrio, G., and Villarreal, P., 2001, J. Chem. Phys., 115, 10438.

Lawley, K. P., 1988, Chem. Phys., 127, 363.

Lawley, K. P., and Donovan, R. J., 1993, J. Chem. Soc. Faraday Trans., 89, 1885.

Lawrence, W. G., van Marter, T. A., Nowlin, M. L., and Heaven, M. C., 1997, J. Chem. Phys., 106, 127.

Lenzer, T., Furlanetto, M. R., Asmis, K. R., and Neumark, D. M., 1998, J. Chem. Phys., 109, 10754. 
Lenzer, T., Yourshaw, I., Furlanetto, M. R., Reiser, G., and Neumark, D. M., 1999, J. Chem. Phys., 110, 9578.

Lepetit, B., Roncero, O., Buchachenko, A. A., and Halberstadt, N., 2002, J. Chem. Phys., 116, 8367.

Levy, D. H., 1981, Adv. Chem. Phys., 47, 323.

Li, Z., Zadoyan, R., Apkarian, V. A., and Martens, C. C., 1995, J. Phys. Chem., 99, 7453.

Lienau, C., and Zewail, A. H., 1994, Chem. Phys. Lett., 222, 224.

Lienau, C., and Zewail, A. H., 1996, J. Phys. Chem., 100, 18629.

Liu, L., and Guo, H., 1995, Chem. Phys. Lett., 237, 299.

Liu, Q., Wang, J., and Zewail, A. H., 1993, Nature, 364, 427.

Liu, Q., Wang, J., and Zewail, A. H., 1995, J. Phys. Chem., 99, 11321.

Ma, Z., Jons, S. D., Giese, C. F., and Gentry, W. R., 1991, J. Chem. Phys., 94, 8608.

Macler, M., and Heaven, M. C., 1991, Chem. Phys., 151, 219.

Martin, F., Bacis, R., Churassy, S., and Vergés, J., 1986, J. Mol. Spectrosc., 116, 71.

Meier, C., Engel, V., and Beswick, J., 1998, Chem. Phys. Lett., 287, 487.

Miller, W. H., 1991, Phys. Reports, 199, 73.

Miranda, M. P., Beswick, J. A., and Halberstadt, N., 1994, Chem. Phys., 187, 185.

Mulliken, R. S., 1957, Phys. Rev., 57, 500. 
Mulliken, R. S., 1971, J. Chem. Phys., 55, 288.

Nakagawa, K., Kitamura, M., Suzuki, K., Kondow, T., Munakata, T., and Kasuya, T., 1986, Chem. Phys., 106, 259.

Naumkin, F. Y., 1991, Sov. Phys. Lebedev Inst. Reports (USA), 8, 27.

Naumkin, F. Y., 1998, Chem. Phys., 226, 319.

Naumkin, F. Y., 2001, Chem. Phys. Chem. [Angew. Chem. 40], 2, 121.

Naumkin, F. Y., and Knowles, P. J., 1995, J. Chem. Phys., 103, 3392.

Naumkin, F. Y., and McCourt, F., 1997, J. Chem. Phys., 107, 5702.

Naumkin, F. Y., and McCourt, F., 1998a, J. Chem. Phys., 108, 9301.

Naumkin, F. Y., and McCourt, F., 1998b, Chem. Phys. Lett., 292, 63.

Naumkin, F. Y., and McCourt, F., 1998c, Chem. Phys. Lett., 294, 71.

Naumkin, F. Y., and McCourt, F., 1999, Mol. Phys., 96, 1043.

Noorbatcha, I., Raff, L. M., and Thomson, D. L., 1984, J. Chem. Phys., 81, 5658.

Nowlin, M. L., and Heaven, M. C., 1993, J. Chem. Phys., 99, 5654.

Papanikolas, J. M., Vorsa, V., Nadal, M. E., Campagnola, P. J., Gord, J. R., and Lineberger, W. C., 1992, J. Chem. Phys., 97, 7002.

Papanikolas, J. M., Vorsa, V., Nadal, M. E., Campagnola, P. J., Gord, J. R., and Lineberger, W. C., 1993, J. Chem. Phys., 99, 8733.

Partridge, H., Stallcop, J. R., and Levine, E., 2001, J. Chem. Phys., 115, 6471. 
Pazyuk, E. A., Stolyarov, A. V., Pupyshev, V. I., Stepanov, N. F., Umanski, S. Y., and Buchachenko, A. A., 2001, Mol. Phys., 99, 91.

Pedersen, D. B., and Weitz, E., 2002, J. Chem. Phys., 116, 9897.

Philippoz, J. M., Monot, R., and van den Bergh, H., 1986, Helv. Phys. Acta, 58, 1089.

Philippoz, J. M., van den Bergh, H., and Monot, R., 1987, J. Phys. Chem., 91, 2545.

Philippoz, J. M., Monot, R., and van Den Bergh, H., 1990, J. Chem. Phys., 92, 288.

Prosmiti, R., Cunha, C., Villarreal, P., and Delgado-Barrio, G., 2002a, J. Chem. Phys., 116, 9249.

Prosmiti, R., Villarreal, P., and Delgado-Barrio, G., 2002b, Chem. Phys. Lett., $359,473$.

Prosmiti, R., Villarreal, P., Delgado-Barrio, G., and Roncero, O., 2002c, Chem. Phys. Lett., 359, 229.

Rock, A. B., van Zoeren, C. M., Kable, S. H., Edvards, G. B., and Knight, A. E. W., 1988, J. Chem. Phys., 89, 6777.

Rohrbacher, A., Janda, K. C., Beneventi, L., Casavecchia, P., and Volpi, G. G., 1997a, J. Phys. Chem., A101, 6528.

Rohrbacher, A., Williams, J., Janda, K. C., Cybulski, S. M., Burcl, R., SzczesŚniak, M. M., Chąasiński, G., and Halberstadt, N., 1997b, J. Chem. Phys., $106,2685$. 
Rohrbacher, A., Ruchti, T., Janda, K. C., Buchachenko, A. A., Hernández, M. I., González-Lezana, T., Villarreal, P., and Delgado-Barrio, G., 1999a, J. Chem. Phys., 110, 256.

Rohrbacher, A., Williams, J., and Janda, K. C., 1999b, Phys. Chem. Chem. Phys., 1,5263 .

Roncero, O., and Gray, S. K., 1996, J. Chem. Phys., 104, 4999.

Roncero, O., Campos-Martínez, J., Cortina, A. M., Villarreal, P., and DelgadoBarrio, G., 1988, Chem. Phys. Lett., 148, 62.

Roncero, O., Villarreal, P., Delgado-Barrio, G., Halberstadt, N., and Janda, K. C., 1993, J. Chem. Phys., 99, 1035.

Roncero, O., Halberstadt, N., and Beswick, J. A., 1994a, in Reaction Dynamics in Clusters and Condensed Phases, edited by J. Jortner, R. D. Levine, and B. Pullmann (Kluwer Academic Publishers), p. 73.

Roncero, O., Halberstadt, N., and Beswick, J. A., 1994b, Chem. Phys. Lett., 226, 82.

Roncero, O., Halberstadt, N., and Beswick, J. A., 1996, J. Chem. Phys., 104, 7554.

Roncero, O., Caloto, D., , Janda, K. C., and Halberstadt, N., 1997, J. Chem. Phys., 107, 1406.

Roncero, O., Campos-Martínez, J., Hernández, M. I., Delgado-Barrio, G., VilLarreal, P., and Rubayo-Soneira, J., 2001a, J. Chem. Phys., 115, 2566.

Roncero, O., Lepetit, B., Beswick, J. A., Halberstadt, N., and Buchachenko, A. A., 2001b, J. Chem. Phys., 115, 6961. 
Rössler, F., 1935, Z. Phys., 96, 251.

Rubinson, M., and Steinfeld, J. I., 1974, Chem. Phys., 4, 467.

Rubinson, M., Garetz, B., and Steinfeld, J., 1974a, J. Chem. Phys., 60, 3082.

Rubinson, M., Garetz, B., and Steinfeld, J. I., 1974b, J. Chem. Phys., 60, 3082.

Saenger, K. L., McClelland, G. M., and Herschbach, D. R., 1981, J. Phys. Chem., 85,3333 .

Schek, I., Jortner, J., Raz, T., and Levine, R. D., 1996, Chem. Phys. Lett., 257, 273.

Schröder, H., and Gabriel, H., 1996, J. Chem. Phys., 104, 587.

Schroeder, J., and Troe, J., 1987, Annu. Rev. Phys. Chem., 38, 163.

Schwartz, B. J., King, J. C., Zhang, J. Z., and Harris, C. B., 1993, Chem. Phys. Lett., 203, 503.

Secrest, D., and Eastes, W., 1972, J. Chem. Phys., 56, 2502.

Selwyn, J. E., and Steinfeld, J. I., 1969, Chem. Phys. Lett., 4, 217.

Sharfin, W., Johnson, K. E., Wharton, L., and Levy, D. H., 1979, J. Chem. Phys., $71,1292$.

Smalley, R. E., Levy, D. H., and Wharton, L., 1976, J. Chem. Phys., 64, 3266.

Smalley, R. E., Wharton, L., and Levy, D. H., 1978, J. Chem. Pys., 68, 671.

Starovoitov, E. M., 1990, Izv. Vysshikh Uchebnykh Zavedenii, Khim. Khim. Tekhnol. (Bulletin of Highest Education Institutions, Chem. and Chem. Technol.), 33, 41.

Steinfeld, J. I., 1984, J. Phys. Chem. Ref. Data, 13, 445. 
Steinfeld, J. I., 1987, J. Phys. Chem. Ref. Data, 16, 903.

Steinfeld, J. I., and Klemperer, W., 1965, J. Chem. Pys., 42, 3475.

Stevens Miller, A. E., Chuang, C., Fu, H. C., Higgins, K. F., and Klemperer, W., 1999, J. Chem. Phys., 111, 7844.

Sulkes, M., Tusa, J., and Rice, S. A., 1980, J. Chem. Phys., 72, 5733.

TaO, F.-M., and Klemperer, W., 1992, J. Chem. Phys., 97, 440.

Teichteil, C., and PÉlissier, M., 1994, Chem. Phys., 180, 1.

Tellinghuisen, J., 1982, J. Chem. Phys., 76, 4736.

Tellinghuisen, J., 1985, J. Chem. Phys., 82, 4012.

Teule, R., Stolte, S., and Urbachs, W., 1999, Laser Chem., 18, 111.

Thayer, C. A., and Yardley, J. T., 1972, J. Chem. Phys., 57, 3992.

Thommen, F., Evard, D. D., and Janda, K. C., 1985, J. Chem. Phys., 74, 163.

Tscherbul, T. V., Zaitsevskit, A. V., Buchachenko, A. A., and Stepanov, N. F., in press, Russ. J. Phys. Chem., in press.

Tully, J., 1977, in Semiempirical Methods in Electronic Structure Calculations, edited by G. A. Segal (Plenum Press, New York), pp. Part A, 199.

Urbachs, W., Aben, I., Milan, J. B., Somsen, J., Stuiver, A. G., and Hogervorst, W., 1993, Chem. Phys., 184, 285.

Uzer, T., 1991, Phys. Reports, 199, 73.

Valentini, J. J., and Cross, J. B., 1982, J. Chem. Phys., 77, 572. 
Villarreal, P., Miret-Artés, S., Roncero, O., Delgado-Barrio, G., Beswick, J. A., Halberstadt, N., and Coalson, R. D., 1991, J. Chem. Phys., 94, 4230.

Wan, C., Gupta, M., Baskin, J. S., Kim, Z. H., and Zewail, A. H., 1997, J. Chem. Phys., 106, 4353.

Willberg, D. M., Gutmann, M., Breen, J. J., and Zewail, A. H., 1992, J. Chem. Phys., 96, 198.

Williams, J., Rohrbacher, A., Djahandideh, D., Janda, K. C., Jamka, A., Tao, F., and Halberstadt, N., 1997, Mol. Phys., 91, 573.

Williams, J., Rohrbacher, A., Seong, J., Maranayagam, N., Janda, K. C., Burcl, R., Szczesśniak, M. M., Chazasiński, G., Cybulski, S. M., and Halberstadt, N., 1999, J. Chem. Phys., 111, 997.

Wood, R. W., 1911a, Philos. Mag. (ser. 6), 21, 309.

Wood, R. W., 1911b, Philos. Mag. (ser. 6), 22, 469.

Xu, Y., Jäger, W., Ozier, I., and Gerry, M. C. L., 1993, J. Chem. Phys., 98, 3726.

Yourshaw, I., ZhaO, Y., and Neumark, D. M., 1996, J. Chem. Phys., 105, 351.

Yourshaw, I., Lenzer, T., Reiser, G., and Neumark, D. M., 1998, J. Chem. Phys., $109,5247$.

Zadoyan, R., Li, Z., Ashjian, P., Martens, C. C., and Apkarian, V. A., 1994a, Chem. Phys. Lett., 218, 504.

Zadoyan, R., Li, Z., Martens, C. C., and Apkarian, V. A., 1994b, J. Chem. Phys., 101,6648 . 
Zadoyan, R., Sterling, M., and Apkarian, V. A., 1996, J. Chem. Soc. Faraday Trans., $92,1821$.

Zadoyan, R., Almy, J., and Apkarian, V. A., 1997, Faraday Discuss., 108, 255.

Zamith, S., Meier, C., Halberstadt, N., and Beswick, J. A., 1999, J. Chem. Phys., $110,960$.

ŽĎÁnská, P., ČEk, P. S., and Jungwirth, P., 2000, J. Chem. Phys., 112, 10761.

Zewail, A. H., Dantus, M., Bowman, R. M., and Mokhtari, A., 1992, J. Photochem. Photobiol. A, 62, 301.

Zhao, M., and Rice, S. A., 1992, J. Chem. Phys., 96, 7583.

Zhao, Y., Yourshaw, I., Reiser, G., Arnold, C. C., and Neumark, D. M., 1994, J. Chem. Phys., 101, 6538. 


\section{Table captions}

Table 1 : Equilibrium distances $R_{e}(\AA)$ and binding energies $D_{e}\left(\mathrm{~cm}^{-1}\right)$ of the T-shaped and linear minima for selected $\mathrm{Ar} \cdots \mathrm{I}_{2}$ PES of the $X$ and $B$ electronic states. For experimental data from Levy's and Klemperer's groups (cited as Levy and Klemperer), approximate estimations of $D_{e}$ from $D_{0}$ are given with the error bars of the $D_{0}$ values. PP stands for pairwise potential.

Table 2 : Dissociation energies $D_{e}$ and $D_{0}\left(\mathrm{~cm}^{-1}\right)$ of the T-shaped and linear isomers of the $\operatorname{Ar} \cdots \mathrm{I}_{2}(X, B)$ complex from experimental data and selected PES.

Table 3 : Symmetry correlations for the $B\left({ }^{3} \Pi 0_{u}^{+}\right)$and crossing states of the $\mathrm{I}_{2}$ molecule and the $\mathrm{Ar} \cdots \mathrm{I}_{2}$ complex in the linear $\left(C_{\infty v}\right)$, T-shaped $\left(C_{2 v}\right)$, and bent $\left(C_{s}\right)$ configurations. Asterisks indicate the crossing states coupled to $B$ within the IDIM model. 
Table 1:

\begin{tabular}{|c|c|c|c|c|c|c|}
\hline \multirow[t]{2}{*}{ Entry } & \multirow[t]{2}{*}{ State } & \multirow[t]{2}{*}{ Potential or data } & \multicolumn{2}{|c|}{ T-shaped } & \multicolumn{2}{|c|}{ Linear } \\
\hline & & & $R_{e}$ & $D_{e}$ & $R_{e}$ & $D_{e}$ \\
\hline 1 & $X$ & $\begin{array}{l}\text { PP from Rg-Rg' interactions } \\
\text { (Secrest and Eastes, 1972) }\end{array}$ & 4.52 & 362.8 & - & - \\
\hline 2 & $X$ & $\begin{array}{l}\text { Naumkin-Knowles DIM PT1 } \\
\text { (Naumkin and Knowles, 1995) }\end{array}$ & 3.93 & 230.1 & 5.13 & 209.6 \\
\hline 3 & $X$ & $\begin{array}{l}\text { IDIM } \\
\text { (Buchachenko and Stepanov, 1996b) }\end{array}$ & 3.88 & 254.6 & - & - \\
\hline 4 & $X$ & $\begin{array}{l}\text { IDIM PT1 } \\
\text { (Buchachenko and Stepanov, 1996b) }\end{array}$ & 3.88 & 254.5 & - & - \\
\hline 5 & $X$ & $\begin{array}{l}\text { DIM } \\
\text { (Naumkin, 1998) }\end{array}$ & 3.93 & 230.5 & 5.13 & 209.5 \\
\hline 6 & $X$ & $\begin{array}{l}\text { DIM PT1 (TP2) } \\
\text { (Buchachenko et al., 2000b) }\end{array}$ & 3.93 & 233.1 & 5.19 & 189.0 \\
\hline 7 & $X$ & $\begin{array}{l}\text { DIM PT1 (dJVN2) } \\
\text { (Buchachenko et al., 2000b) }\end{array}$ & 3.94 & 230.2 & 5.15 & 201.9 \\
\hline 8 & $X$ & $\begin{array}{l}\text { Ab initio MP2 } \\
\text { (Kunz et al., 1998) }\end{array}$ & 3.95 & 234.4 & 5.12 & 256.7 \\
\hline 9 & $X$ & $\begin{array}{l}\text { Ab initio MP4 } \\
\text { (Kunz et al., 1998) }\end{array}$ & 4.14 & 187.4 & 5.15 & 205.8 \\
\hline 10 & $X$ & $\begin{array}{l}\text { Ab initio } \operatorname{CCSD}(\mathrm{T}) \\
\text { (Kunz et al., 1998) }\end{array}$ & 4.16 & 179.2 & 5.16 & 192.5 \\
\hline 11 & $X$ & $\begin{array}{l}\text { Ab initio CCSD-T } \\
\text { (Naumkin and McCourt, 1998c) }\end{array}$ & 4.22 & 143.4 & 5.32 & 151.5 \\
\hline 12 & $X$ & $\begin{array}{l}\text { Ab initio CCSD-T RECP } \\
\text { (Naumkin, 2001) }\end{array}$ & 4.02 & 203.1 & 5.09 & 224.4 \\
\hline 13 & $X$ & $\begin{array}{l}\text { Ab initio CCSD }(\mathrm{T}) \mathrm{RECP} \\
\text { (Prosmiti et al., 2002b) }\end{array}$ & 3.96 & 235.4 & 5.05 & 268.3 \\
\hline 14 & $X$ & $\begin{array}{l}\text { Experimental, Levy } \\
\text { (Blazy et al., 1980) }\end{array}$ & - & $250 \pm 3$ & - & - \\
\hline 15 & $X$ & $\begin{array}{l}\text { Experimental, Klemperer } \\
\text { (Stevens Miller et al., 1999) }\end{array}$ & $4.0 \pm 0.4$ & $166 \pm 15$ & - & $196 \pm 15$ \\
\hline 16 & $B$ & $\begin{array}{l}\text { PP, collision data } \\
\text { (Rubinson et al., 1974b) }\end{array}$ & 4.24 & 223.8 & - & - \\
\hline 17 & $B$ & $\begin{array}{l}\text { Empirical PP } \\
\text { (Beswick and Jortner, 1978b) }\end{array}$ & 3.93 & 200.0 & - & - \\
\hline 18 & $B$ & $\begin{array}{l}\text { PP, spectroscopic data } \\
(\text { Gray, 1992) }\end{array}$ & 3.92 & 244.0 & - & - \\
\hline 19 & $B$ & $\begin{array}{l}\text { IDIM } \\
\text { (Buchachenko and Stepanov, 1996b) }\end{array}$ & 3.83 & 248.8 & - & - \\
\hline 20 & $B$ & $\begin{array}{l}\text { IDIM PT1 } \\
\text { (Buchachenko and Stepanov, 1996b) }\end{array}$ & 3.82 & 248.9 & - & - \\
\hline 21 & $B$ & $\begin{array}{l}\text { DIM } \\
\text { (Naumkin, 1998) }\end{array}$ & 3.82 & 248.0 & 5.54 & 144.7 \\
\hline 22 & $X$ & $\begin{array}{l}\text { Experimental, Levy } \\
\text { (Blazy et al., 1980) }\end{array}$ & - & $236 \pm 3$ & - & - \\
\hline 23 & $X$ & $\begin{array}{l}\text { Experimental, Klemperer } \\
\text { (Stevens Miller et al., } 19983\end{array}$ & - & $140 \pm 15$ & - & - \\
\hline
\end{tabular}


Table 2:

\begin{tabular}{|c|c|c|c|c|c|c|}
\hline \multirow[t]{2}{*}{ State } & \multirow[t]{2}{*}{ Potential or data } & \multirow[t]{2}{*}{ Ref. } & \multicolumn{2}{|c|}{ T-shaped } & \multicolumn{2}{|c|}{ Linear } \\
\hline & & & $D_{e}$ & $D_{0}$ & $\bar{D}_{e}$ & $D_{0}$ \\
\hline$X$ & DIM PT1 & (Buchachenko et al., 2000b) & 233 & 209 & 189 & 166 \\
\hline$X$ & $\operatorname{CCSDT}(\mathrm{T}) \mathrm{RECP}$ & (Prosmiti et al., 2002b) & 235 & 212 & 268 & 238 \\
\hline$X$ & Levy, experiment & (Blazy et al., 1980) & 250 & $237 \pm 3$ & - & - \\
\hline$X$ & Klemperer, experiment & (Stevens Miller et al., 1999) & 166 & $142 \pm 15$ & 196 & $172 \pm 1.5$ \\
\hline$B$ & DIM PT1 & (Buchachenko and Stepanov, 1996b) & 249 & 222 & - & - \\
\hline$B$ & Gray's PP & $($ Gray, 1992) & 244 & 222 & - & - \\
\hline$B$ & Levy, experiment & (Blazy et al., 1980) & 236 & $223 \pm 3$ & - & - \\
\hline$B$ & Klemperer, experiment & (Stevens Miller et al., 1999) & 140 & $128 \pm 15$ & - & - \\
\hline
\end{tabular}

Table 3:

\begin{tabular}{|c|c|c|c|c|c|}
\hline \multirow[t]{2}{*}{ State } & \multicolumn{2}{|c|}{$\mathrm{I}_{2}$ molecule } & \multicolumn{3}{|c|}{ Ar $\cdots \mathrm{I}_{2}$ complex } \\
\hline & $\Omega_{w}^{\sigma}$ & $D_{\infty h}$ & $C_{\infty v}$ & $C_{2 v}$ & $C_{s}$ \\
\hline$B$ & $0_{u}^{+}$ & $\Sigma_{u}^{+}$ & $\Sigma^{+}$ & $B_{2}$ & $A^{\prime}$ \\
\hline$B^{\prime \prime}$ & $1_{u}$ & $\Pi_{u}$ & $\Pi$ & $A_{1} \oplus B_{1}$ & $A^{\prime \prime} \oplus A^{\prime *}$ \\
\hline$a$ & $1_{g}$ & $\Pi_{g}$ & $\Pi$ & $A_{2} \oplus B_{2}^{*}$ & $A^{\prime \prime} \oplus A^{\prime *}$ \\
\hline$a^{\prime}$ & $0_{g}^{+}$ & $\Sigma_{g}^{+}$ & $\Sigma^{+*}$ & $A_{1}$ & $A^{\prime *}$ \\
\hline & $3_{u}$ & $\Phi_{u}^{9}$ & $\Phi$ & $A_{1} \oplus B_{1}$ & $A^{\prime \prime} \oplus A^{\prime *}$ \\
\hline 1 & $2_{g}$ & $\Delta_{g}$ & $\Delta$ & $A_{1} \oplus B_{1}$ & $A^{\prime \prime} \oplus A^{\prime *}$ \\
\hline & $0_{u}^{-}$ & $\Sigma_{u}^{-}$ & $\Sigma^{-}$ & $A_{2}$ & $A^{\prime \prime}$ \\
\hline
\end{tabular}




\section{Figure captions}

Figure 1 : Potential energy curves of the $B$ and crossing valence states of molecular iodine. The zero for energies is the lowest $\mathrm{I}\left({ }^{2} \mathrm{P}_{3 / 2}\right)+\mathrm{I}\left({ }^{2} \mathrm{P}_{3 / 2}\right)$ dissociation limit.

Figure 2 : Contour plots of the $X$ and $B$ PES of the Ar $\cdots \mathrm{I}_{2}$ complex. The molecular frame is defined such that the I-I vector lies along the abcissa axis, its norm being the internuclear distance at equilibrium. $x$ and $y$ are the cartesian coordinates of the argon atom in this molecular frame. Left column : DIM PT1 (TP2) potentials for the $X$ and $B$ states. Right column : ab initio $X$ potential and empirical $B$ potential. Ten contour lines are equally spaced from -200 to $0 \mathrm{~cm}^{-1}$.

Figure 3 : Contour plots of the diabatic coupling matrix elements between the $B\left({ }^{3} \Pi 0_{u}^{+}\right)$ and crossing states of the Ar $\cdots \mathrm{I}_{2}$ complex computed using the DIM PT1 method in Jacobi $(R, \theta)$ coordinates. The abcissa is the angle $\theta$ between the Jacobi vectors ( $\mathbf{R}$ from Ar to the center of mass of $\mathrm{I}_{2}, \mathbf{r}$ linking the I atoms). The ordinate is the distance $R$ between Ar and the center of mass of $\mathrm{I}_{2}$. The $\mathrm{I}_{2}$ distance is taken as the equilibrium value for the $B$ state. Contour lines are drawn from -39 to $39 \mathrm{~cm}^{-1}$ with a step of $6 \mathrm{~cm}^{-1}$, dashed lines correspond to negative values (Reprinted from ref. (Lepetit et al., 2002)).

Figure 4 : Final vibrational distributions of $\mathrm{I}_{2}(B)$ after excitation of the $\mathrm{Ar} \cdots \mathrm{I}_{2}$ Van der Waals complex with wavelengths of 476.5, 488, and $496.5 \mathrm{~nm}$. - results from the 3-dimension wave packet calculation (Zamith et al., 1999); $\diamond$ experimental results from Philippoz et al. (1987). (Reprinted from (Zamith et al., 1999)).

Figure 5 : Spectrum associated to the initial state $\operatorname{Ar} \cdots \mathrm{I}_{2}\left(B, v^{\prime}=21, n^{\prime}=0\right)$ with zero total angular momentum: the points correspond to numerical time-independent calculations, the solid and dashed lines to an analytical radiationless model based on three zero-order bound states. Magnification by a factor of 10 gives a better view of the details. (Reprinted from (Roncero and Gray, 1996)).

Figure 6 : Vibrational population of the $\mathrm{I}_{2}(B)$ fragment as a function of time for the initial state $\operatorname{Ar} \cdots \mathrm{I}_{2}\left(B, v^{\prime}=21, n^{\prime}=0\right)$, zero total angular momentum : a) numerical time-dependent results, b) analytical model with adjusted parameters. (Reprinted from (Roncero and Gray, 1996)).

Figure 7 : Norm of the autocorrelation function for $\operatorname{Ar} \cdots \mathrm{Cl}_{2}\left(B, v^{\prime}=18, n^{\prime}=0\right)$. a) $\mathrm{J}=0$. The insert gives the details at short times. The solid line is the result of a wavepacket calculation, the dashed line corresponds to an analytical fit of the spectrum in terms of independent resonances b) $J=15, K=0$ (solid line) and $K=14$ (dashed line). (Reprinted from ref. (Roncero et al., 1997)).

Figure 8 : Top panels : Contour plots of the amplitude densities of the lowest bound levels of $\operatorname{Ar}_{\cdots} \mathrm{I}_{2}(X, J=0)$ computed at equilibrium $\mathrm{I}_{2}$ distance for the DIM PT1 potential. 
Dashed lines correspond to negative amplitudes. Abcissae and ordinates are defined as in Fig. 2. Zero energy corresponds to the $\mathrm{Ar}+\mathrm{I}_{2}(X, v=0)$ dissociation limit. Bottom panels : Contour plots of the amplitude densities of $\operatorname{Ar} \cdots \mathrm{I}_{2}(B, v=21, J=0)$ states (even permutation symmetry of the I nuclei). Dashed lines correspond to negative amplitudes. Abcissae and ordinates are defined as in Fig. 2. Zero energy corresponds to the $\mathrm{Ar}+$ $\mathrm{I}_{2}(B, v=21)$ dissociation limit. (Reprinted from (Roncero et al., 2001b)).

Figure 9 : The $\left(B, v, J=0^{++}\right) \leftarrow\left(X, v=0, J=1^{--}\right)$Ar $\cdots \mathrm{I}_{2}$ absorption spectra calculated for the T-shaped (top panel) and linear (bottom panel) isomers using the DIM PT1 PES. The \pm superscripts refer to the parity (with respect to inversion of the coordinates) and permutation symetry (with respect to the exchange of the iodine atoms) of the initial or final states. Note the difference in scale for the absorption cross sections from the T-shaped and linear isomers. (Reprinted from (Roncero et al., 2001b)).

Figure 10 : Ratio of absorption cross sections of the linear and T-shaped isomers for the DIM PT1 PES. (Reprinted from ref. (Roncero et al., 2001b)).

Figure 11 : Curve labelled relative intensity : relative intensity of the fluorescence excitation spectrum of Ar $\cdots \mathrm{I}_{2}$ as a function of the vibrational state $v^{\prime}$ of $\mathrm{I}_{2}$ that was originally excited. The original data from Levy (1981) has been multiplied by a factor of 10 to match the other results. Curve labelled relative quantum yield : LIF intensity divided by absorbance from Goldstein et al. (1986). Curve labelled vibrational predissociation efficiency : Relative quantum yield corrected for the Franck-Condon factors for $\mathrm{I}_{2}$ absorption in $v^{\prime}$ and $\mathrm{I}_{2}$ emission in $v^{\prime}-3$, from Burke and Klemperer (1993a).

Figure 12 : Calculated $v s$ experimental rates for the electronic predissociation of $\operatorname{Ar} \cdots \mathrm{I}_{2}\left(B, v^{\prime}\right)$, $v^{\prime}=16$ to 24 . The results were scaled such that the electronic predissociation linewidth coincides with the experimental one for $v^{\prime}=20$. The black dots represent the experimental data of Burke and Klemperer (1993a). The empty dots represent the results of a threedimensional wave-packet calculation for electronic predissociation by the $a 1_{g}$ state. The continuous and dashed lines correspond to an attractive and a repulsive Van der Waals interaction in the $a 1_{g}$ state, respectively. (Reprinted from (Roncero et al., 1996)).

Figure 13 : Predissociation rates (in $\mathrm{ns}^{-1}$ ) as a function of the initial vibrational excitation $v^{\prime}$, for the ground Van der Waals level. Two results from three-dimension wave packet calculations are shown : $k_{E P+V P}$ : full calculation, where the $B\left({ }^{3} \Pi 0_{u}^{+}\right)$potential energy surface is coupled to the four dissociative states $B^{\prime \prime} 1_{u}, a 1_{g}, a^{\prime} 0_{g}^{+}$and $12 g . k_{V P}$ : only the $B\left({ }^{3} \Pi 0_{u}^{+}\right)$state is included in the calculation, EP cannot take place. Also shown is the experimental total rate from Burke and Klemperer (1993a). This rate has been extrapolated from vibrational predissociation efficiencies VPE by assuming quasi-linear dependence of $k_{V P}$ as a function of $v^{\prime}$. Only the $v^{\prime}=18$ and 21 rates result from direct measurements (Breen et al., 1990; Willberg et al., 1992). (Reprinted from ref. (Lepetit et al., 2002)).

Figure 14 : Energy diagram and couplings of the simplified analytical model for the 
classification of IVR regimes. Zero-order bound states are coupled together by a constant value $V$. One of them is the bright state and can be populated by photo-excitation, the others are dark states. We assume that diagonalization of this zero-order hamiltonian provides first-order eigenstates with equidistant eigenenergies seperated by $\Delta$. These eigenstates are coupled to the $\alpha$ and $\beta$ continua, which gives them some width $\Gamma$ which is assumed to be constant. (Reprinted from (Roncero et al., 1997)).

Figure 15 : Spectra corresponding to the situation described in Fig. 14. The coupling between zero-order states is fixed to $V=1$, and the spacing between first-order states is $\Delta=1.5$. One gradually moves from sparse to intermediate and then to statistical IVR regimes as the coupling strength of the first-order states to the continuum is increased from $\Gamma=0.05$ to 0.5 and then 5 . (Reprinted from (Roncero et al., 1997)).

Figure 16 : Time evolution of the initial state (zero-order bright state) for the three IVR regimes of Fig. 15. (Reprinted from (Roncero et al., 1997)). 
Figure 1: Potential energy curves of the $B$ and crossing valence states of molecular iodine.

The zero for energies is the lowest $\mathrm{I}\left({ }^{2} \mathrm{P}_{3 / 2}\right)+\mathrm{I}\left({ }^{2} \mathrm{P}_{3 / 2}\right)$ dissociation limit. 

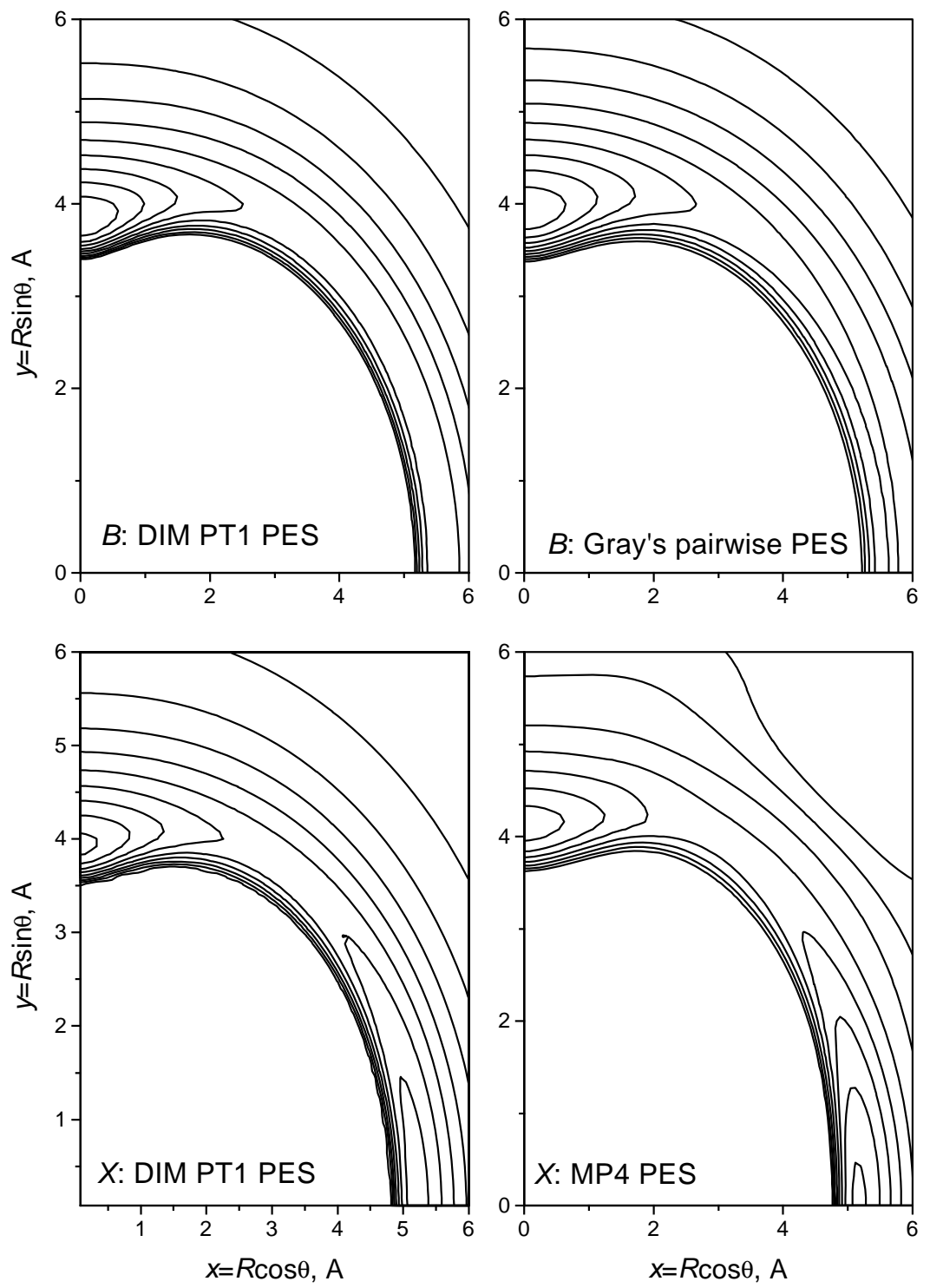

Figure 2: Contour plots of the $X$ and $B$ PES of the Ar $\cdots \mathrm{I}_{2}$ complex. The molecular frame is defined such that the I-I vector lies along the abcissa axis, its norm being the internuclear distance at equilibrium. $x$ and $y$ are the cartesian coordinates of the argon atom in this molecular frame. Left column : DIM PT1 (TP2) potentials for the $X$ and $B$ states. Right column : ab initio $X$ potential and empirical $B$ potential. Ten contour lines 89 are equally spaced from -200 to $0 \mathrm{~cm}^{-1}$. 

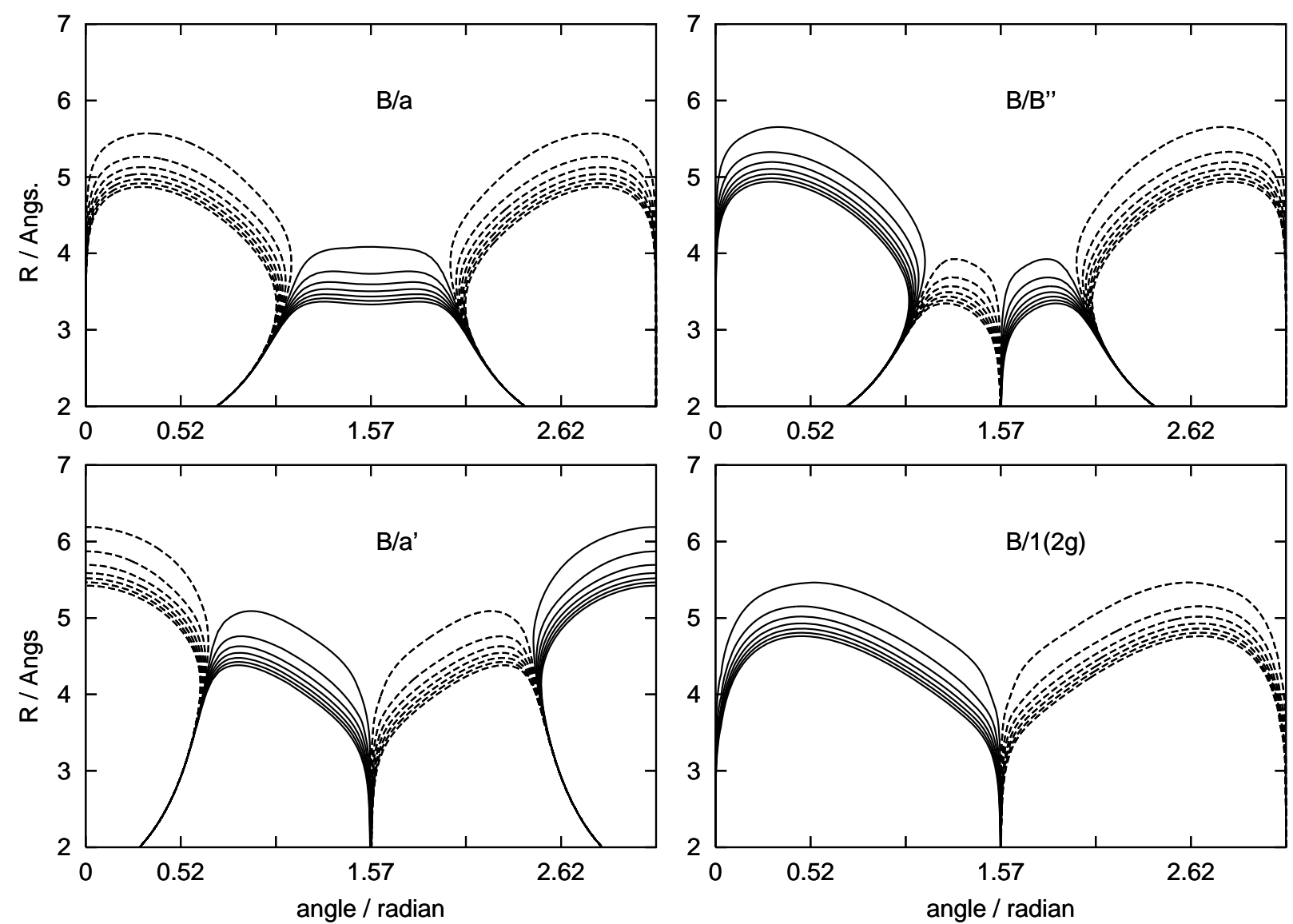

Figure 3: Contour plots of the diabatic coupling matrix elements between the $B\left({ }^{3} \Pi 0_{u}^{+}\right)$ and crossing states of the Ar $\cdots \mathrm{I}_{2}$ complex computed using the DIM PT1 method in Jacobi $(R, \theta)$ coordinates. The abcissa is the angle $\theta$ between the Jacobi vectors $(\mathbf{R}$ from Ar to the center of mass of $\mathrm{I}_{2}, \mathbf{r}$ linking the I atoms). The ordinate is the distance $R$ between Ar and the center of mass of $\mathrm{I}_{2}$. The $\mathrm{I}_{2}$ distance is taken as the equilibrium value for the $B$ state. Contour lines are drawn from -39 to $39 \mathrm{~cm}^{-1}$ with a step of $6 \mathrm{~cm}^{-1}$, dashed lines correspond to negative values (Reprinted from ref. Lepetit et al. (2002)). 


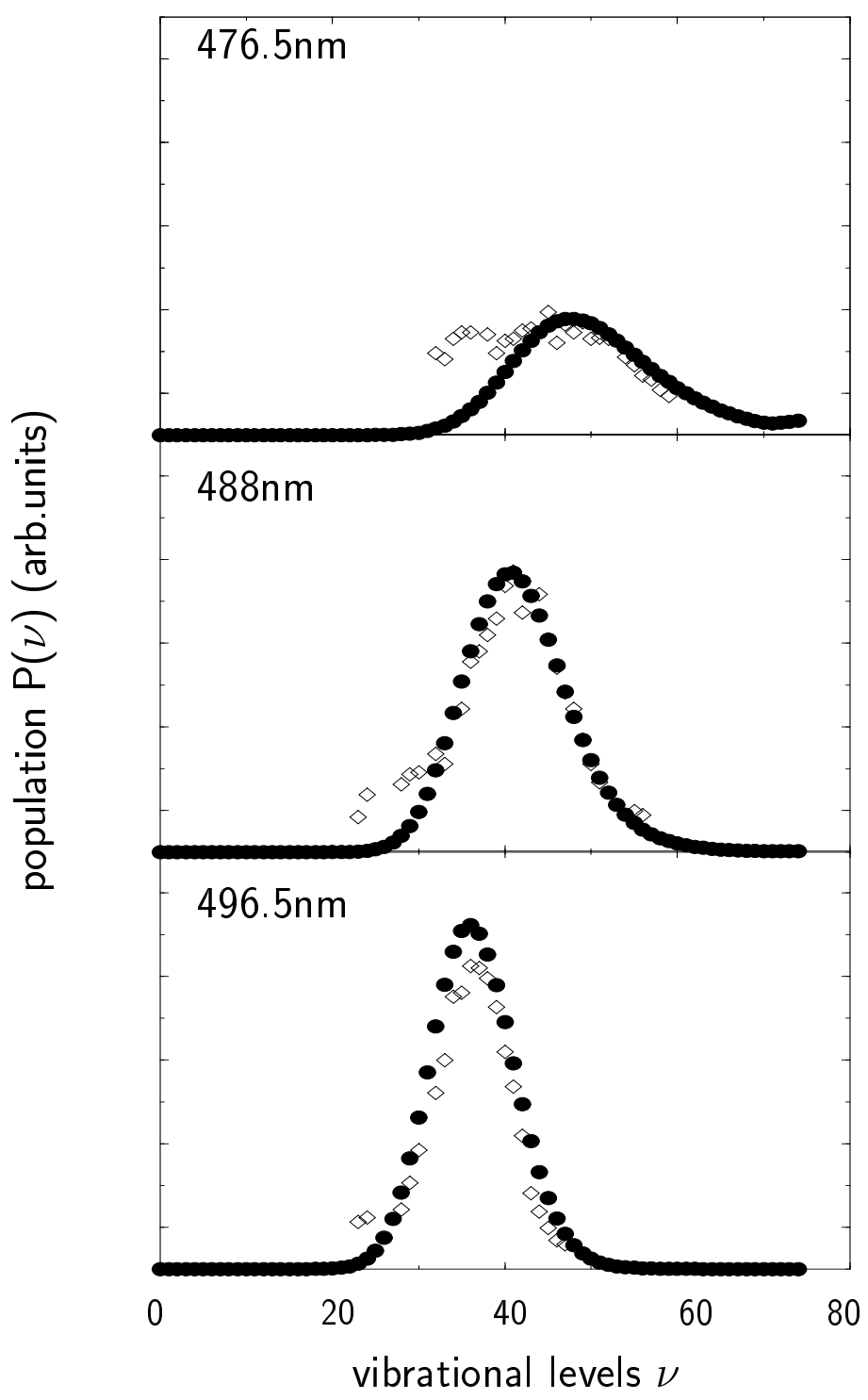

Figure 4: Final vibrational distributions of $\mathrm{I}_{2}(B)$ after excitation of the Ar $\cdots \mathrm{I}_{2}$ Van der Waals complex with wavelengths of $476.5,488$, and $496.5 \mathrm{~nm}$. • results from the 3dimension wave packet calculation (Zamith et al., 1999); $\diamond$ experimental results from Philippoz et al. (1987). (Reprinted from (Zgmith et al., 1999)). 


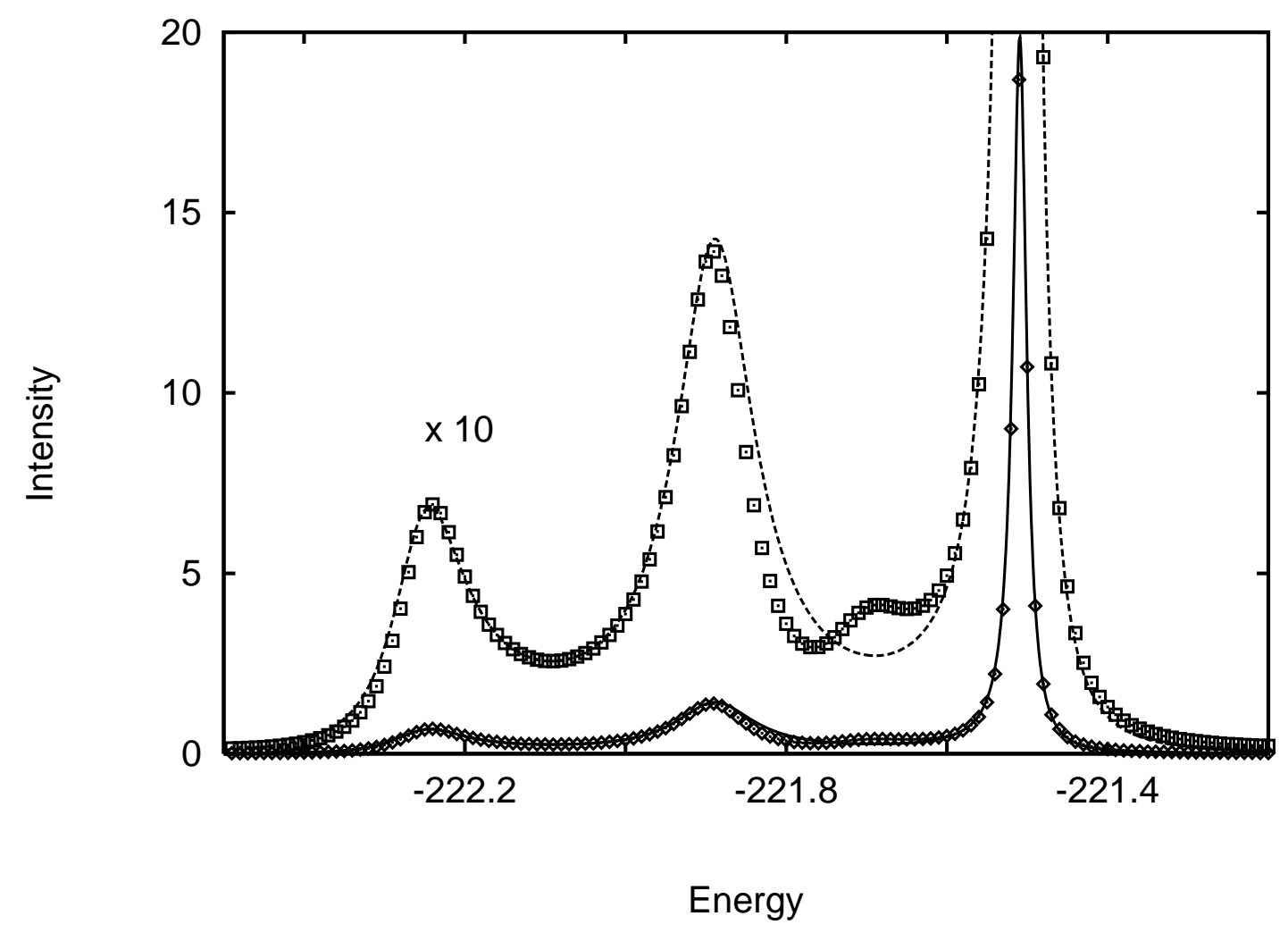

Figure 5: Spectrum associated to the initial state $\operatorname{Ar}_{\cdots} \mathrm{I}_{2}\left(B, v^{\prime}=21, n^{\prime}=0\right)$ with zero total angular momentum: the points correspond to numerical time-independent calculations, the solid and dashed lines to an analytical radiationless model based on three zero-order bound states. Magnification by a factor of 10 gives a better view of the details. (Reprinted from (Roncero and Gray, 1996)). 

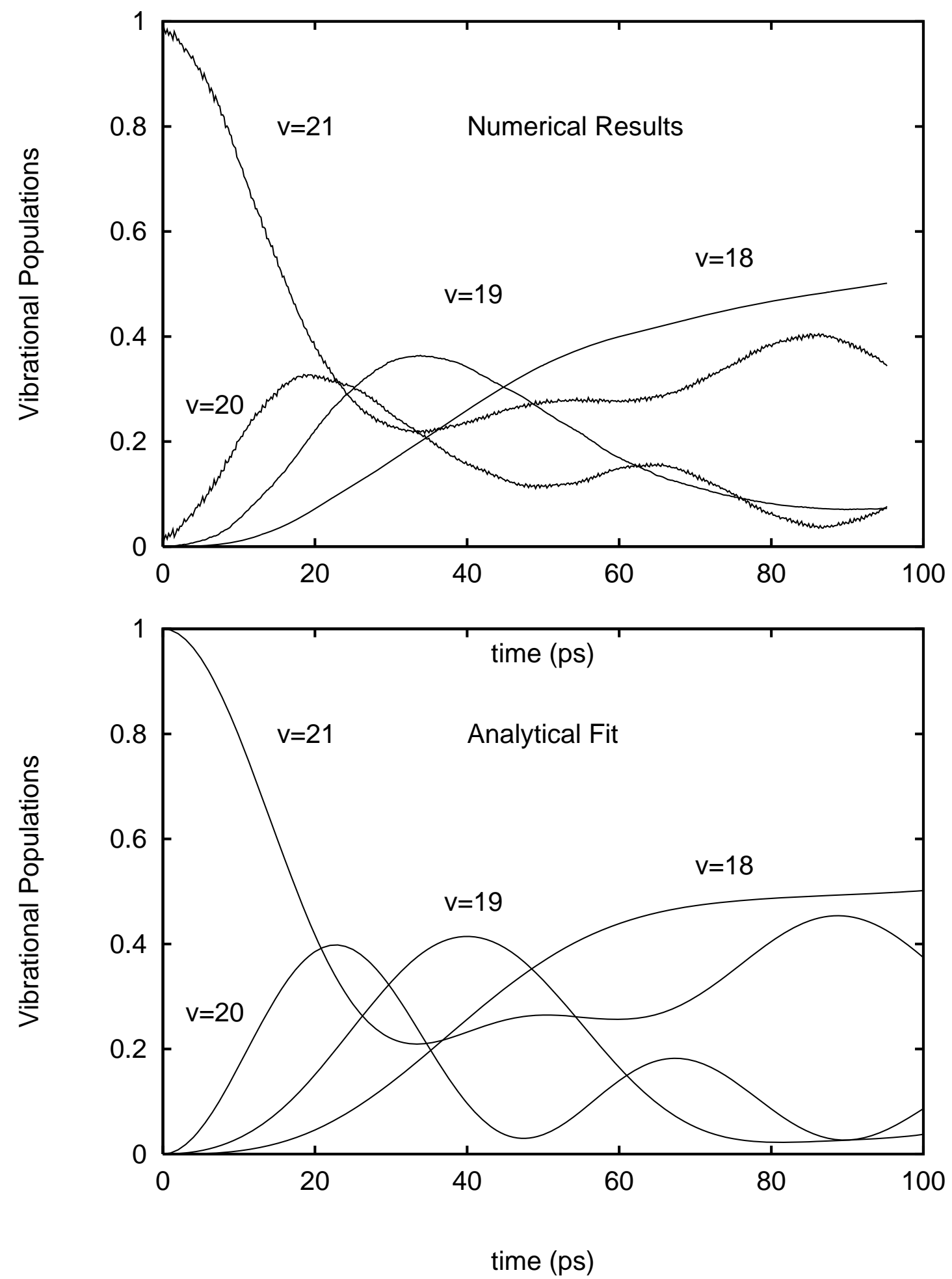

Figure 6: Vibrational population of the $\mathrm{I}_{2}(B)$ fragment as a function of time for the initial state $\operatorname{Ar} \cdots \mathrm{I}_{2}\left(B, v^{\prime}=21, n^{\prime}=0\right)$, zero total angular momentum : a) numerical time-dependent results, b) analytical model with adjusted parameters. (Reprinted from (Roncero and Gray, 1996)). 

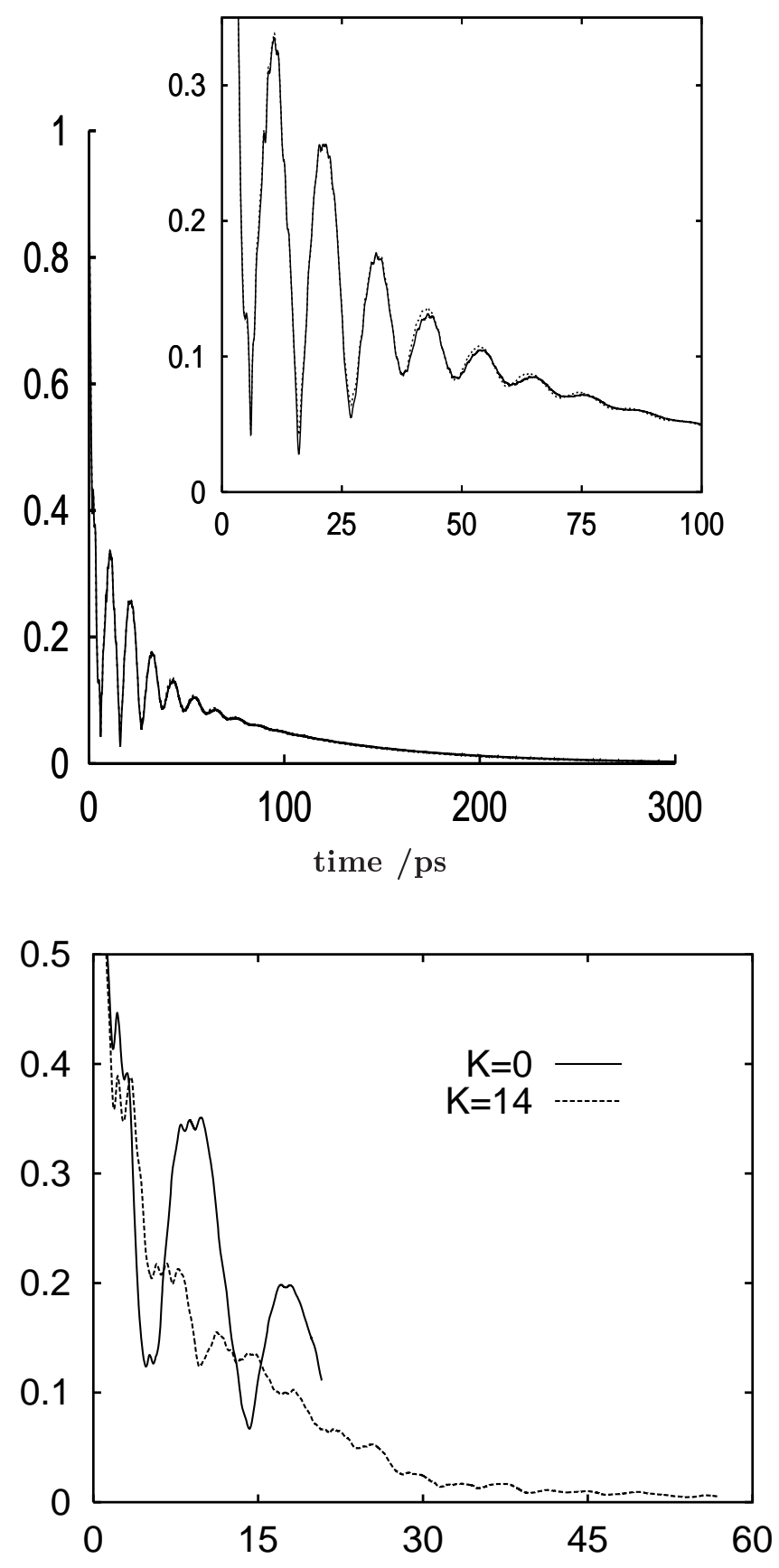

Figure 7: Norm of the autocorrelation function for $\mathrm{Ar} \cdots \mathrm{Cl}_{2}\left(B, v^{\prime}=18, n^{\prime}=0\right)$. a) $\mathrm{J}=0$. The insert gives the details at short times. The solid line is the result of a wavepacket calculation, the dashed line corresponds to an analytical fit of the spectrum in terms of independent resonances b) $J=15, K=0$ (solid line) and $K=14$ (dashed line). (Reprinted from ref. (Roncero et al., 1997)9. 


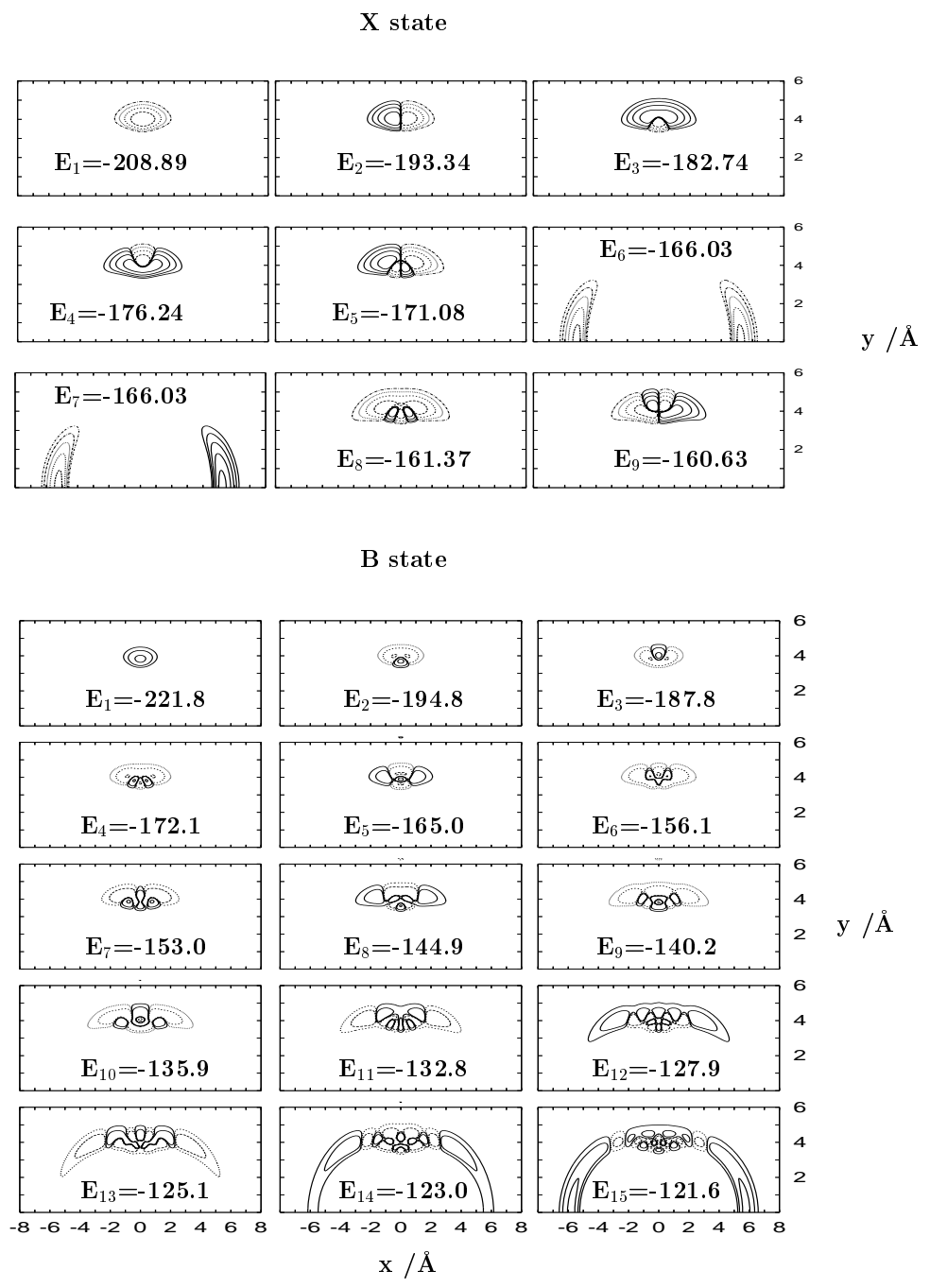

Figure 8: Top panels : Contour plots of the amplitude densities of the lowest bound

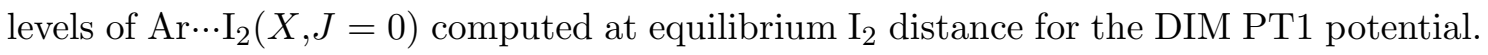
Dashed lines correspond to negative amplitudes. Abcissae and ordinates are defined as in Fig. 2. Zero energy corresponds to the $\mathrm{Ar}+\mathrm{I}_{2}(X, v=0)$ dissociation limit. Bottom panels : Contour plots of the amplitude densities of $\operatorname{Ar}_{\cdots} \mathrm{I}_{2}(B, v=21, J=0)$ states (even permutation symmetry of the I nuclei). Dashed lines correspond to negative amplitudes. Abcissae and ordinates are defined as in Fig. 2. Zero energy corresponds to the Ar + $\mathrm{I}_{2}(B, v=21)$ dissociation limit. (Reprinted from (Roncero et al., 2001b)). 

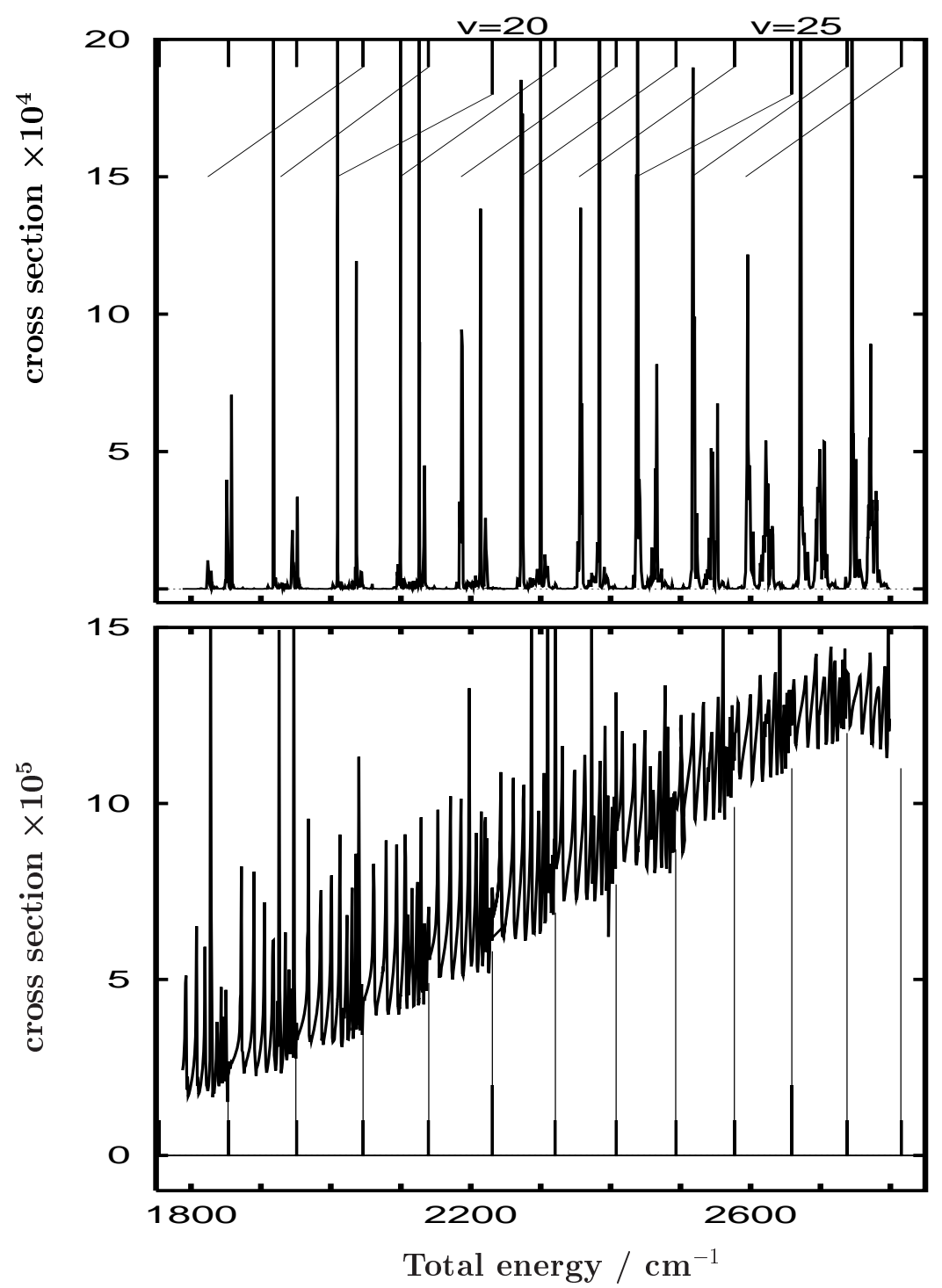

Figure 9: The $\left(B, v, J=0^{++}\right) \leftarrow\left(X, v=0, J=1^{--}\right)$Ar $\cdots \mathrm{I}_{2}$ absorption spectra calculated for the T-shaped (top panel) and linear (bottom panel) isomers using the DIM PT1 PES. The \pm superscripts refer to the parity (with respect to inversion of the coordinates) and permutation symetry (with respect to the exchange of the iodine atoms) of the initial or final states. Note the difference in scale for the absorption cross sections from the 96

T-shaped and linear isomers. (Reprinted from (Roncero et al., 2001b)). 


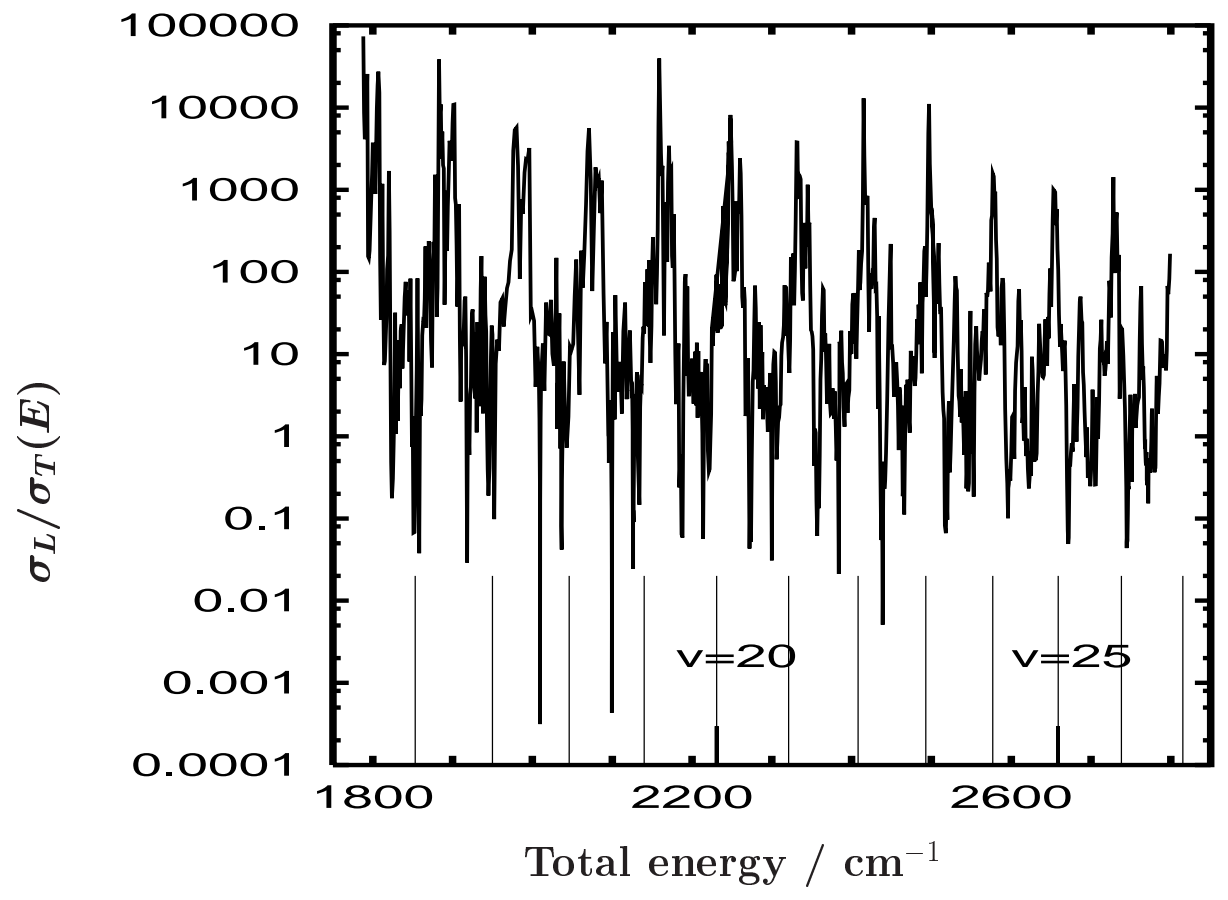

Figure 10: Ratio of absorption cross sections of the linear and T-shaped isomers for the DIM PT1 PES. (Reprinted from ref. (Roncero et al., 2001b)). 


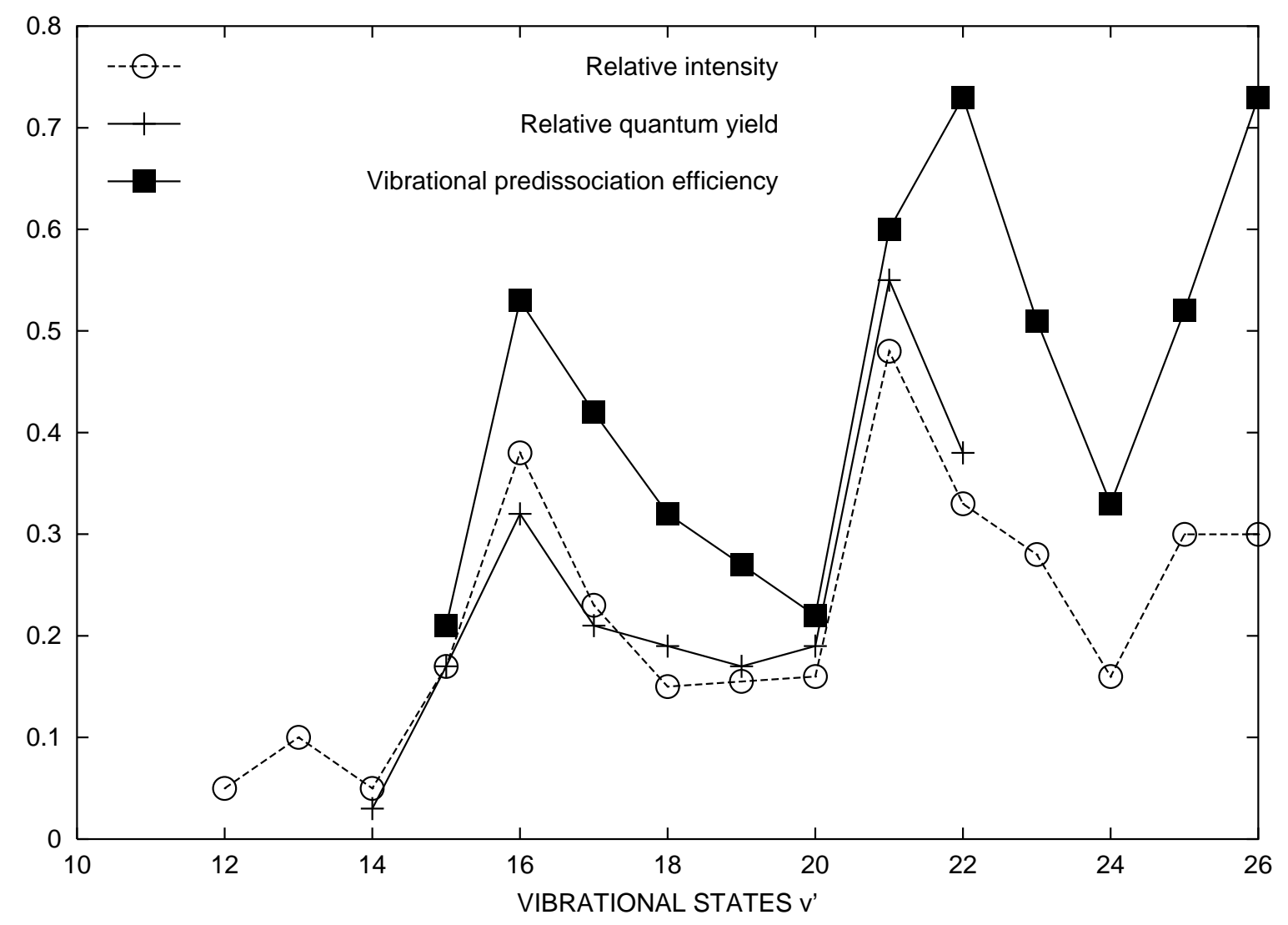

Figure 11: : Curve labelled relative intensity : relative intensity of the fluorescence excitation spectrum of $\mathrm{Ar} \cdots \mathrm{I}_{2}$ as a function of the vibrational state $v^{\prime}$ of $\mathrm{I}_{2}$ that was originally excited. The original data from Levy (1981) has been multiplied by a factor of 10 to match the other results. Curve labelled relative quantum yield : LIF intensity divided by absorbance from Goldstein et al. (1986). Curve labelled vibrational predissociation efficiency : Relative quantum yield corrected for the Franck-Condon factors for $\mathrm{I}_{2}$ absorption in $v^{\prime}$ and $\mathrm{I}_{2}$ emission in $v^{\prime}-3$, from Burke and Klemperer (1993a). 


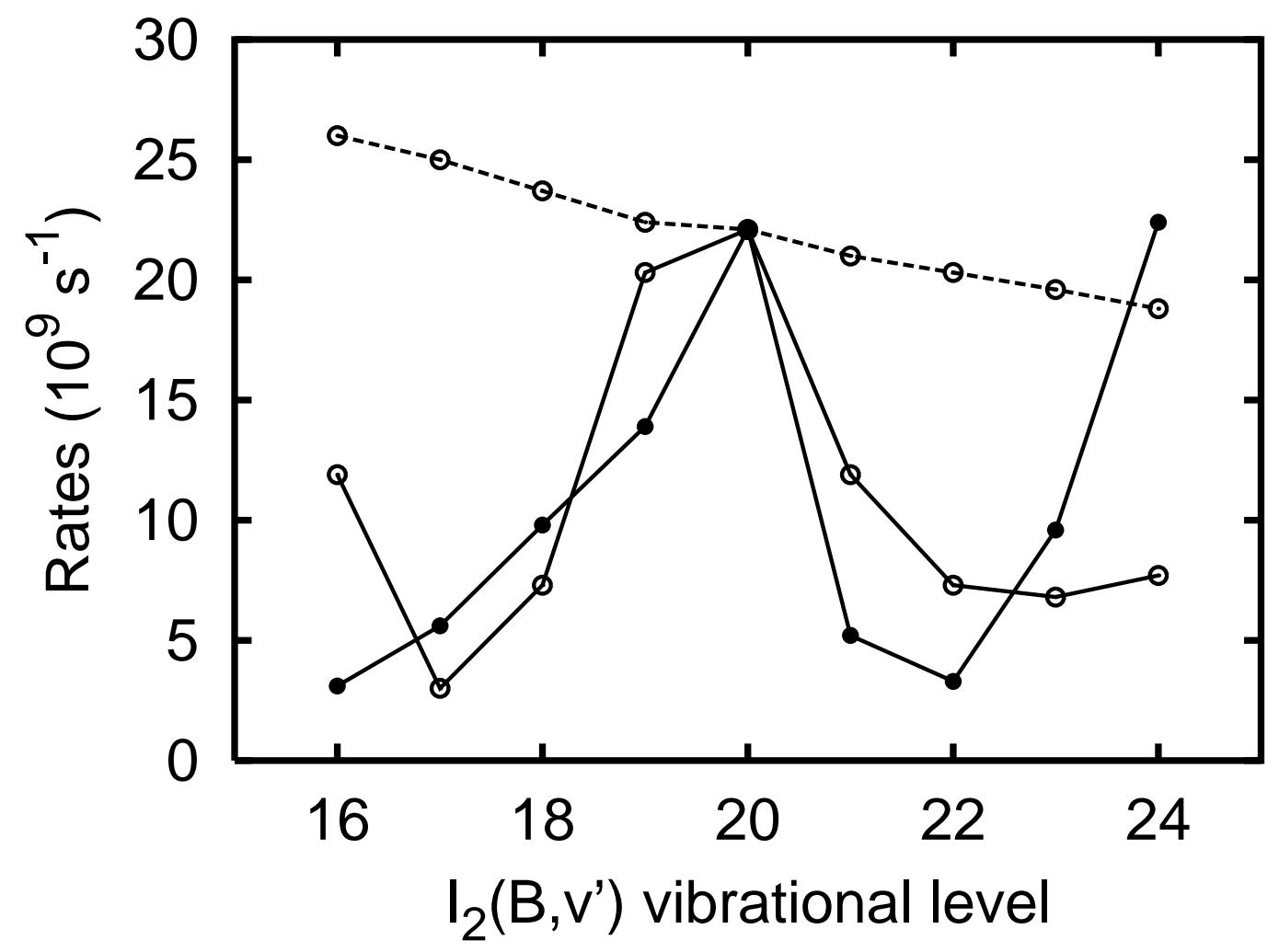

Figure 12: : Calculated vs experimental rates for the electronic predissociation of $\operatorname{Ar} \cdots \mathrm{I}_{2}\left(B, v^{\prime}\right), v^{\prime}=16$ to 24 . The results were scaled such that the electronic predissociation linewidth coincides with the experimental one for $v^{\prime}=20$. The black dots represent the experimental data of Burke and Klemperer (1993a). The empty dots represent the results of a three-dimensional wave-packet calculation for electronic predissociation by the $a 1_{g}$ state. The continuous and dashed lines correspond to an attractive and a repulsive Van der Waals interaction in the $a 1_{g}$ state, respectively. (Reprinted from (Roncero et al., 1996)). 


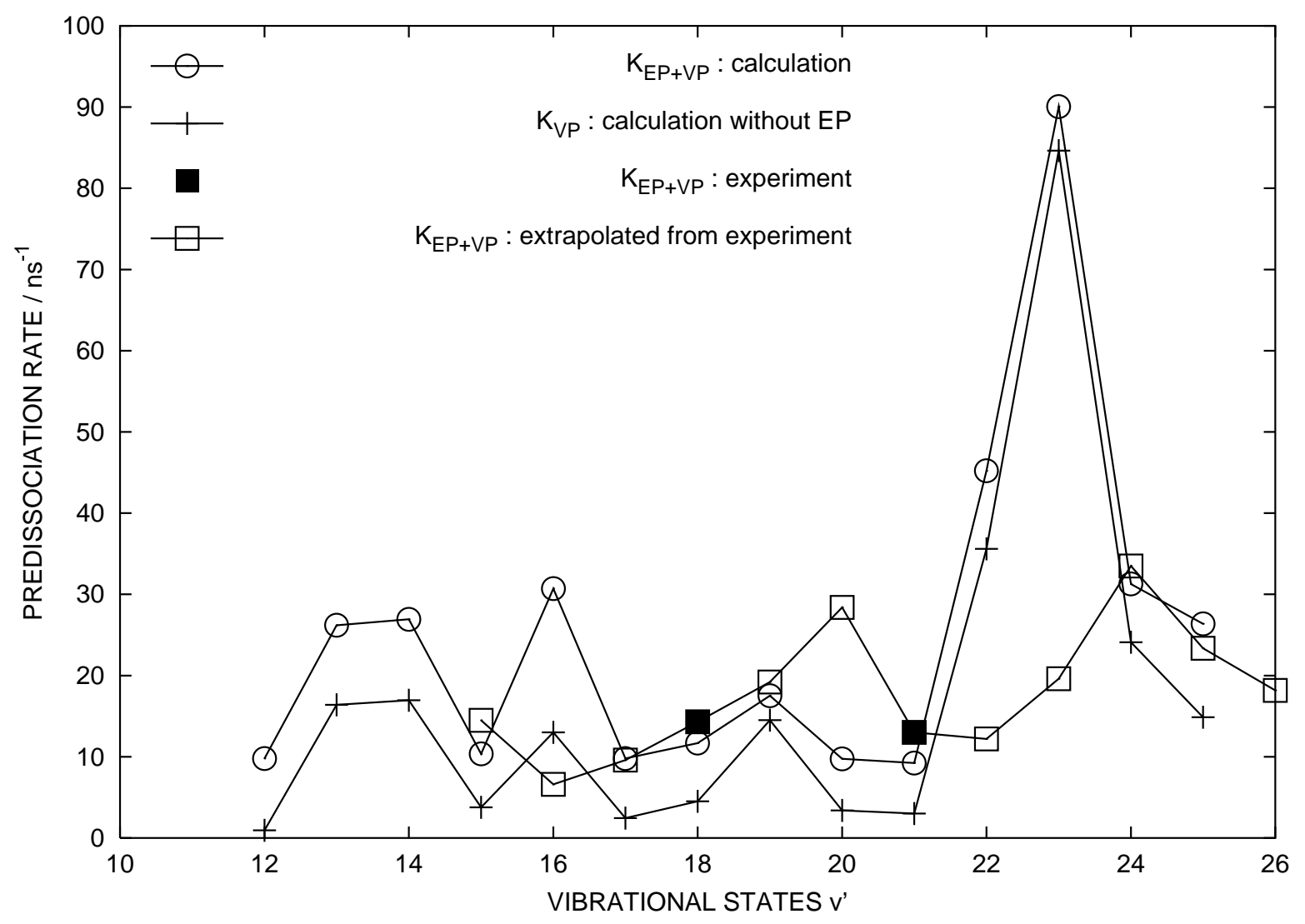

Figure 13: : Predissociation rates $\left(\mathrm{in}_{\mathrm{ns}}{ }^{-1}\right.$ ) as a function of the initial vibrational excitation $v^{\prime}$, for the ground Van der Waals level. Two results from three-dimension wave packet calculations are shown : $k_{E P+V P}$ : full calculation, where the $B\left({ }^{3} \Pi 0_{u}^{+}\right)$potential energy surface is coupled to the four dissociative states $B^{\prime \prime} 1_{u}, a 1_{g}, a^{\prime} 0_{g}^{+}$and $12 g . k_{V P}$ : only the $B\left({ }^{3} \Pi 0_{u}^{+}\right)$state is included in the calculation, EP cannot take place. Also shown is the experimental total rate from Burke and Klemperer (1993a). This rate has been extrapolated from vibrational predissociation efficiencies VPE by assuming quasi-linear dependence of $k_{V P}$ as a function of $v^{\prime}$. Only the $v^{\prime}=18$ and 21 rates result from direct measurements (Breen et al., 1990; Willberg et al., 1992). (Reprinted from ref. (Lepetit et al., 2002)). 


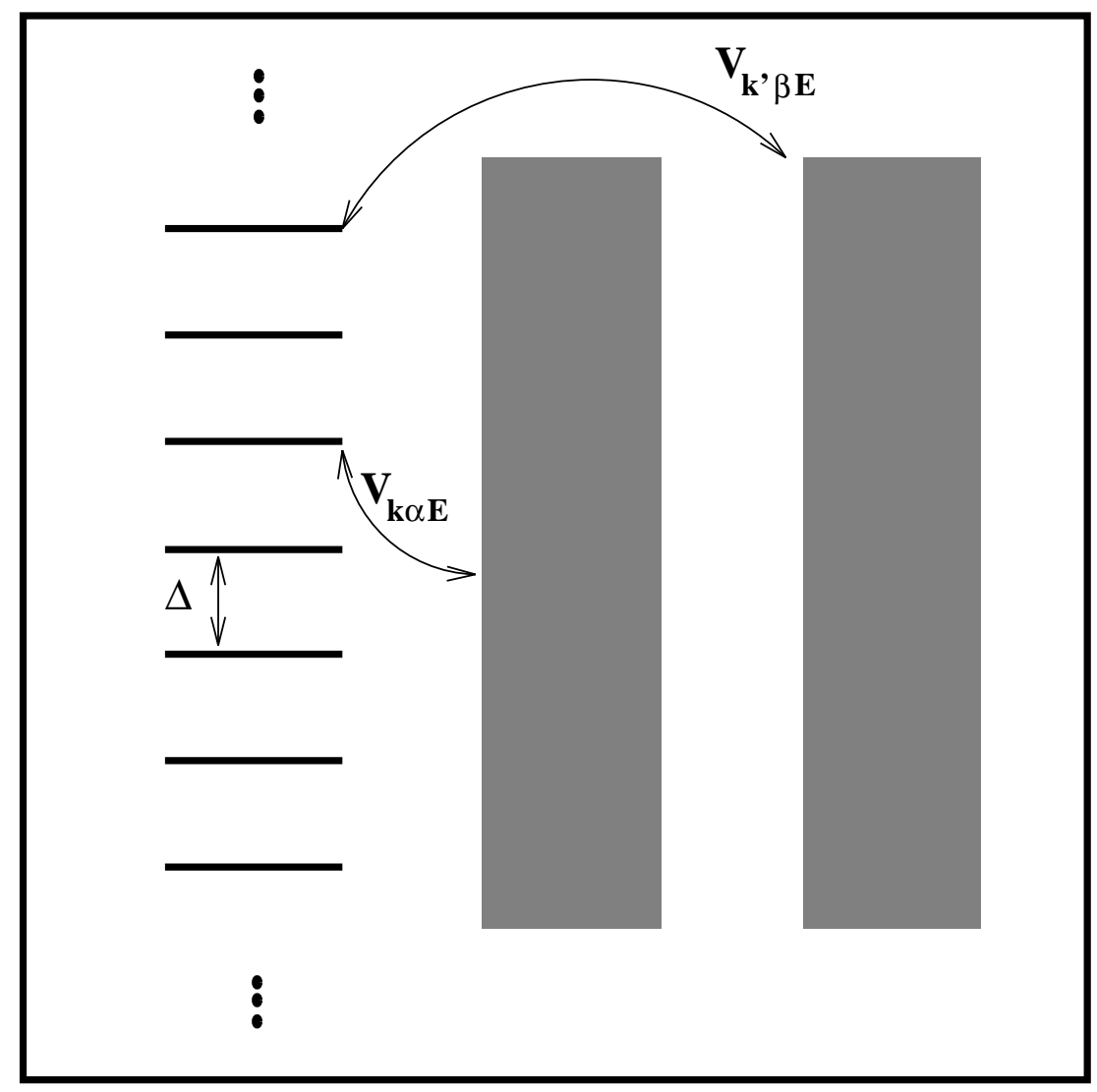

Figure 14: Energy diagram and couplings of the simplified analytical model for the classification of IVR regimes. Zero-order bound states are coupled together by a constant value $V$. One of them is the bright state and can be populated by photo-excitation, the others are dark states. We assume that diagonalization of this zero-order hamiltonian provides first-order eigenstates with equidistant eigenenergies seperated by $\Delta$. These eigenstates are coupled to the $\alpha$ and $\beta$ continua, which gives them some width $\Gamma$ which is assumed to be constant. (Reprinted from (Roncero et al., 1997)). 


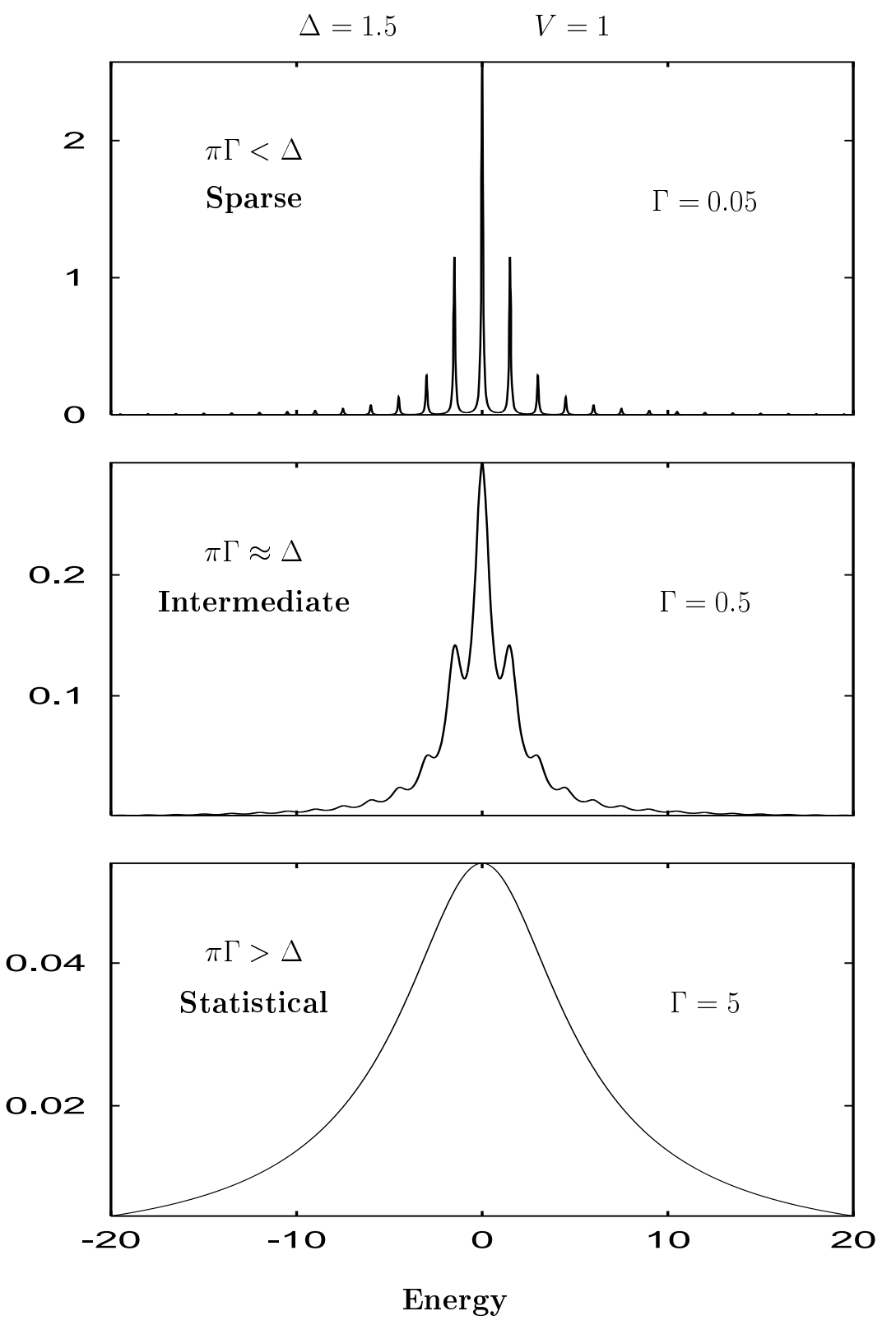

Figure 15: Spectra corresponding to the situation described in Fig. 14. The coupling between zero-order states is fixed to $V=1$, and the spacing between first-order states is $\Delta=1.5$. One gradually moves from sparse to intermediate and then to statistical IVR regimes as the coupling strength of the first-order states to the continuum is increased from $\Gamma=0.05$ to 0.5 and then 5. (Reprinted from (Roncero et al., 1997)). 

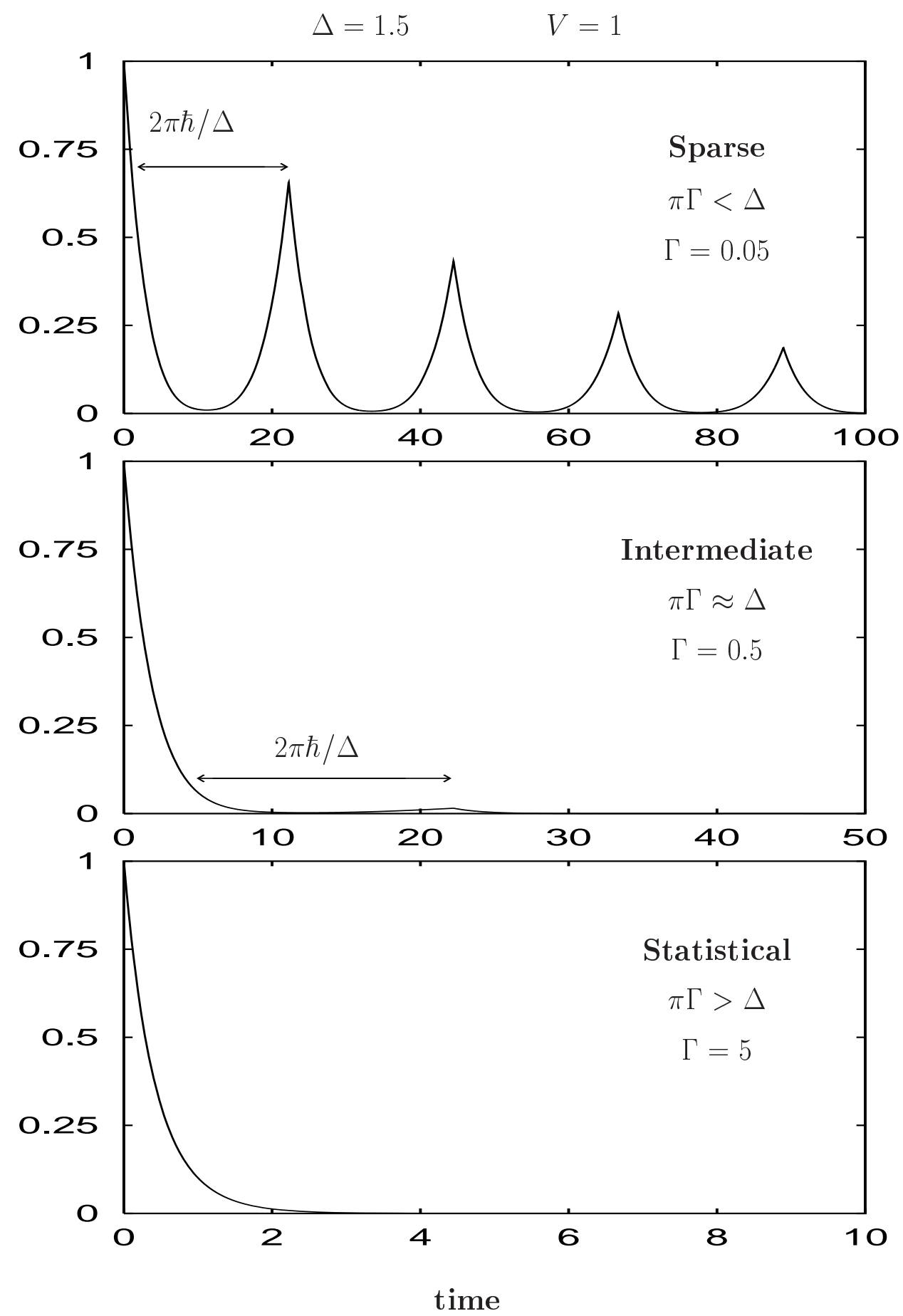

Figure 16: Time evolution of the initial state (zero-order bright state) for the three IVR regimes of Fig. 15. (Reprinted from (Roncero et al., 1997)). 